$\begin{array}{lll}\text { NAME } & : \text { Ronald A。 Andrews } & \text { APPROVED BY: } \\ \text { MAJOR } & : \text { Physics (Solid State) } \\ \text { DEGREE } & : \text { Doctor of Philosophy } \\ \text { DATE OF GRADUATION : December, } 1966 & \end{array}$

DISSERTATION ABSTRACT

OPTICAL AND ELECTRON PARAMAGNETIC RESONANCE

STUDIES OF SODIUM FLUORIDE

EPR studies of $\mathrm{x}$-1rradiated NaF crystals have been made at controlled temperatures below $180^{\circ} \mathrm{K}$ and optical absorption studies of the same samples have been made at room temperature. These studies have been made in order to determine the characteristics of iron as an impurity in $\mathrm{NaF}$ and to investigate the influence of this impurity on the optical bleaching process. To facilitate the controlled temperature experiments, a cryostat has been built and successfully employed In the course of the present work.

The EPR absorption of $x$-irradiated NaF containing fron as an Impurity has distinguished: (i) the F center spectrum, (11) a spectrum 1sotropically centered near $g=2.002$ and with a partially resolved anisotropic structure, and (iii) the $\mathrm{Fe}^{+}$spectrum centered near $g=4.344$. The spectrum near $g=2.002$ is satisfactorily described In terms of a spin Hamiltonian pertaining to an ${ }^{6} \mathrm{~S}_{5 / 2}$ ion located at a lattice site with cubic symmetry and interacting with six equivalent nuclel each with spin $1 / 2$. It is attributed to $\mathrm{Fe}^{3+}$ at a cation site with cubic symmetry. Computer analyses of the observed temperature dependence of the $\mathrm{Fe}^{3+}$ spectrum have indicated that the fine structure constant decreases as the first power in $T$, and the line width Increases as the square root of $\mathrm{T}$, when $\mathrm{T}$ changes from $77^{\circ} \mathrm{K}$ to $180^{\circ} \mathrm{K}$. 


\section{DISCLAIMER}

This report was prepared as an account of work sponsored by an agency of the United States Government. Neither the United States Government nor any agency Thereof, nor any of their employees, makes any warranty, express or implied, or assumes any legal liability or responsibility for the accuracy, completeness, or usefulness of any information, apparatus, product, or process disclosed, or represents that its use would not infringe privately owned rights. Reference herein to any specific commercial product, process, or service by trade name, trademark, manufacturer, or otherwise does not necessarily constitute or imply its endorsement, recommendation, or favoring by the United States Government or any agency thereof. The views and opinions of authors expressed herein do not necessarily state or reflect those of the United States Government or any agency thereof. 


\section{DISCLAIMER}

Portions of this document may be illegible in electronic image products. Images are produced from the best available original document. 
The results are qualitatively consistent with the existing theorles of S-state Ion。 Qualitatively, however, the computed T-dependence of the fine structure constant disagrees substantially with current theories。 An extensive analysis of the $\mathrm{Fe}^{3+}$ spectrum has indicated that the two charge compensating cation vacancles assoclated with each $\mathrm{Fe}^{3+}$ ion are not bound to the $\mathrm{Fe}^{3+}$. This means that the samples which exhibit an EPR signal due to $\mathrm{Fe}^{3+}$ have an excess of cation vacancles, which are free to migrate at room temperature。

By means of optical absorption studies, these samples which exhibit an EPR signal due to $\mathrm{Fe}^{3+}$ (Type I samples) have been compared with samples which show no trace of $\mathrm{Fe}^{3+}$ (Type II samples)。 The results of comparative optical absorption studies indicate that (1) during $x-$ Irradiation. Type II samples color more readily than Type I samples, (2) optical bleaching with F-light is more efficient in Type I samples, and (3) the optical bleaching process exhibits very pronounced "aging" effects in Type I samples. These results are accounted for in terms of a proposed iron impurity controlled mechanism of optical bleaching. This mechanism is based on the formation of cation-anion vacancy pairs, which are attributed to the excess cation vacancies associated with the iron impurity。

The Type I samples also exhibit an unstable optical absorption band on the long wavelength side of the F-band, after extended periods of optical bleaching. This band decays with a time constant $\tau=7.8$ hours. Its observation is made easier by using samples which have a high optical bleaching efficiency. The observed characteristics of this unstable band suggest that it is one of the N-bands of NaF, probably the $\mathrm{N}_{2}$ band。 


$$
\text { C00-1054-30 }
$$

\title{
OPTICAL AND ELECTRON PARAMAGNETIC RESONANCE STUDIES OF SODIUM FLUORIDE
}

by

RONALD A。 ANDREWS

\author{
A DISSERTATION
}

Submitted to the office for Graduate Studies,

Graduate Division of Wayne State University, Detroit, Michigan,

in partial fulfillment of the requirements

for the degree of

DOCTOR OF PHILOSOPHY

1966

MAJOR: PHYSICS (SOLID STATE) APPROVED BY:

Advisor

Date

\section{LEGAL NOTICE}

This report was prepared as an account of Government sponsored work. Nelther the United States, nor the Commission, nor any person acting on behalf of the Commisslon:

A. Makes any warranty or representation, expressed or Implied, with respect to the accuracy, completeness, or usefulness of the information contained in this report, or that the use of any information, apparatus, method, or process disclosed in this report may not infringe privately owned righte; or

B. Assumes any liabilitles with respect to the uae of, or for damages resulting from the use of any information, apparatus, method, or process dlsclosed in thls report. ployee or contractor of the commission, or employee of such contractor, to the extent that such employee or contractor of the Commisslon, or employee of such contractor prepares, disseminates, or provides access to, any information pursuant to his employment or contract with the Commission, or his employment with such contractor. 
The author of this paper wishes to sincerely thank Professor $Y_{0} W_{0}$ Kim for his guidance during the past four years.

Thanks are also due to Messrs. J. VanGemen and $M$. Shopnick for much patience and help in preparing experimental equipment; Miss B。 Collins, Miss J. Filinger, and Mrs. B. Gregory for typing the many drafts of this manuscript; and Mr。 $H_{0}$ Brown for preparing photographs and slides.

The author is also Indebted to the Harshaw Chemical Company for doing a spectroscopic analysis of several crystals of NaF, and the Wayne State University Computing and Data Processing Center for machine time on an IBM 7074 computer.

Sincere appreciation is also expressed to the National Science Foundation for fellowship support during the past four years, and the United States Atomic Energy Commission, whose contract (AT(11-1)-1054) supported the research described herein. 
Page

Acknowledgements. . . . . . . . . . . . . . . . 1 i

List of Tables. . . . . . . . . . . . . . . . v v

List of figures ....................... vi

Chapter

I. Introduction ..................... 1

II. Basic Concepts .. . . . . . . . . . . . 4

Color Centers .. . . . . . . . . . 4

Electron Paramagnet1c Resonance (EPR) . . . . . 11

III. Experimental Set-Up and Techniques . . . . . . . . 17

Optical spectrophotometer .......... 17

EPR Spectrometer. . . . . . . . . . . 22

Helium Cryostat . . . . . . . . . . . 29

IV. Electron Paramagnetic Resonance of $\mathrm{Fe}^{3+}$ in $\mathrm{NaF} . . . .38$

Introduction. . . . . . . . . . . . 38

Experimentals ........................... 39

Experimental Results and Spin Hamiltonian .... 40

Temperature Effects ............. 47

Discussion。. . . . . . . . . . . . . . 53

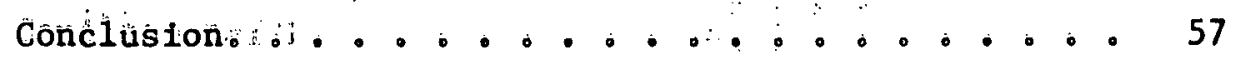

V. Iron Impurity Gontrolled $F$ to $M$ Conversion in

X-Irradiated NaF : . . . . . . . . . . . 59

Introduction.

Experimental Procedure and Results. ........ 61

Empirical Analysis of the Aging Effects....... 69

Proposed Mechanism of Optical Bleaching . . . . . 71 
TABLE OF CONTENTS (Cont'd.)

Chapter

Discussions of the Experimental Results. ...... 76

Conclusion .............. 78

VI。 Observation of an Unstable N Band in NaF。 . . . . 79

Append1x I。 Hyperfine Interaction Term in a Spin Hamiltonian. . 85

Appendix II. Spin Hamiltonian for $\mathrm{S}_{5 / 2}$ - State Ions in a Cubic

Crystalline Field .............. 92

List of References ...................... 102

Autoblographical Statement . . . . . . . . 105 
LIST OF TABLES

Table

Page

1. Spin Hamlltonian parameters for the observed resonance

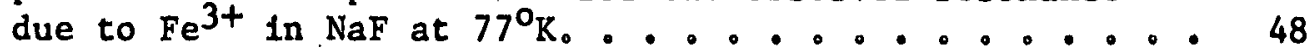

2. Values of Emplrical Parameters of Eq. (28) . . . . . 70 
1. Schematic diagram of an F center: Stereoscopic view plus a section in the (100) plane........... 5

2. (a) Configuration coordinate diagram of an $F$ center

(b) Breathing mode of vibration of the six nearest neighbor cations of the F center.......... 7

3. Schematic diagrams of the current models for F-aggregate centers: (a) A stereoscopic view of the $M$ center and $a$ section in the (100) plane, (b) A stereoscopic view of the $R$ center and a section in the (111) plane, (c) Stereoscopic view of the $\mathrm{N}_{1}$ and $\mathrm{N}_{2}$ centers...... 10

4. Energy level diagram for an electron in a magnetic field. - 13

5. Diagram illustrating the geometry of a sample in a spectrophotometer light beam . . . . . . . . . 18

6. Schematic diagram of an optical absorption spectrophotometer ................. 20

7. Modification of the spectrophotometer sample compartment

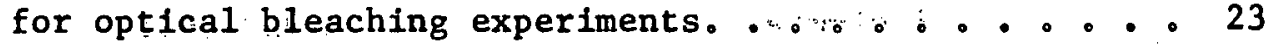

8. Schematic diagram of an EPR spectrometer. . . . . . . 24

9. Output of an EPR spectrometer 1llustrating why the recorded signal is the first derivative of the: absorption line. . 28

10. Scaled schematic drawing of the complete helium cryostat. 30

11. Section of cryostat tall taken at right angles to the section given in Fig. 10 .............. 33

12. Details of the microwave cavity and the coupling mechanism. . . . . . . . . . . . 36

13. The observed EPR spectra (solid lines) and the theoretical fit (dots) of $\mathrm{Fe}^{3+}$ in $\mathrm{NaF}$ at $77^{\circ} \mathrm{K}$ for $\theta=0$, $55^{\circ}$, and

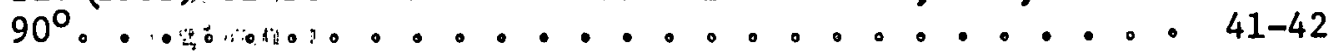

14. The observed EPR spectra (solid lines) and:thertheoretical fit (dots) of $\mathrm{Fe}^{3+}$ in NaF near $180^{\circ} \mathrm{K}$ for $\theta_{n}=90^{\circ}$ and

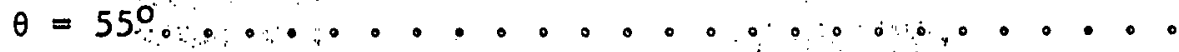


15. The observed temperature dependence of the EPR spectral amplitude for $\theta=90^{\circ}$ and $\theta=55^{\circ}$ (solid curves). The dashed curves represent the experimental curves corrected for the Boltzmann temperature dependence. ...... 50

16. The temperature dependence of $a, \gamma$, and.w found by fitting the experimental data at several temperatures, $g$ and $\delta$ are found to be constant within experimental error...

17. Schematic diagram showing, (a) associated charge compensating cation vacancies in next-nearest neighbor positions forming a "complex", and (b) a charge compensating, vacancles away from the $\mathrm{Fe}^{3+}$. ion. In this case the $\mathrm{Fe}^{3+}$. Ions are left in a site with cubic symmetry and the vacancies are free to migrate at sufficiently high temperatures depending on the activation energy . .

18. Typical x-ray colorability curves for $F$ centers in Type I and Type II samples at room temperature...........

19. Typical optical bleaching curves for the $F$ and $M$ centers in Type $I$ ( $F_{i}$ and $M_{I}$ ) and Type II ( $F_{I I}$ and $M_{I I}$ )

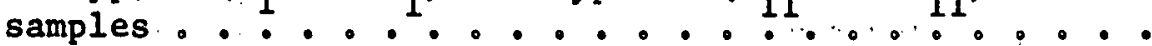

20. A typical M center optical bleaching curve of a Type I sample illustrating aging effects after pauses in the F-light optical bleaching at points $A$ and $B$. The optical density of the M-band is plotted as a function of the net bleaching time. As for the dashed curves, see text.........................

21. Schematic diagram illustrating the models of an anion vacancy $(\alpha)$, a cation vacancy $(\tilde{\alpha})$, a vacancy pair $(\alpha, \tilde{\alpha})$, and the Seitz model of an $M$ center $\left(M_{s}\right) \cdot e^{-}$indicates a trapped electron. .................

22. A probable mode of decay of the $M_{s}$ center into an $M$ center and an $\tilde{a}$ center. $e^{-}$represents a trapped electron. The arrows in:the left hand diagram indicate the probable:motion of lattice lons ...........

23. $\lambda_{\max }$ (dots) of $\mathrm{N}$-bands, and also $\mathrm{F}$ - and $\mathrm{M}$-bands, observed In several alkali halides, and Ivey's relations (straight lines) for the bands. The straight lines, for the $\mathrm{F}-$ and M-bands are taken from ref。 2: $\lambda_{\max }=703 \mathrm{~d} \cdot 1.84$ (F-band) and $\lambda_{\max }=1400, \mathrm{~d}^{1.56}$ (M-band)。 They sare included here for comparison: . . ................

24. Behavior of the band $N$, and the $F$ - and M-bands during optical:bleaching with F-light. Note the difference ordinate scales for the $\mathrm{F}$ - and $\mathrm{M}$-bands and the band $\mathrm{N}$. 
25. Exponential decay with time constant 7.8 hours of the band $\mathrm{N}$, when left in the dark. ........... 83

26. Coordinate system used to find the hyperfine interaction between a paramagnetic ion and a single nuclear magnetic moment. $\vec{H}$ is directed along the $z^{\prime}$ axis and the nuclear magnetic moment is located on the $z$ axis......... 88

27. Coordinate system of the cubic lattice $(x, y, z)$ and a primed coordinate system in which $\vec{H}$ is along the $z^{\prime}$ axis。. . 94

28. Angular variation of the fine structure components of a resonance line for rotation about the [110] axis

$\left(\phi=45^{\circ}\right) \ldots \ldots \ldots \ldots 0$ 
CHAPTER I

\section{INTRODUCTION}

Pure alkali halide crystals are transparent and consist of singly lonlzed alkall ions (positive) and singly charged hallde ions (negative) in a face-centered cubic (space) lattice, except for the cesium halides, which are simple cubic. When subjected to ionizing radiation or made to take on a stochiometric excess of alkall lons, these crystals acquire characteristic coloration ${ }^{1}$ (NaF becomes light orange, $\mathrm{NaCl}$ becomes yellow, KCl becomes violet, etc.). This coloration is due to point defects in the crystalline lattice, which are called color centers. These color centers absorb light from the visible part of the optical spectrum and, in some cases, from the near uitra-violet, depending on the host lattice and the electronic structure of the color centers. When crystals colored by lonizing radiation are exposed to light of the proper frequency, the acquired coloration tends to change, and at the same time the degree of coloration siowly decreases. "Thts is due to the "optical bleaching" of some of the color centers. The bleaching is dual in its operation: selective destruction of one type of color center and formation of other types of color centers. These new color centers exhibit new optical absorption bands which appear in the visible and near infrared parts of the optical spectrum. The bleaching process is found to be affected by the presence of Impurities in the lattice.

A few mechanisms have recently been proposed for the optical bleaching process by several Independent workers. ${ }^{2}$ However, it is not clear which one of the proposed mechanisms gives the correct 
description of the bleaching process. Furthermore, no specific mechanism or mechanisms appear to have been proposed for the role of impurities in the optical bleaching process.

An experimental study of these optical processes and the role of impurities in these processes is the purpose of the present thesis. The experiments described in this thesis make use of iron as an impurity in NaF which is colored by $x$-rays near room temperature. These experiments determine (1) the interaction of $x$-ray photons with the iron impurity and (2) the role of the impurity in the optical bleaching process. The resuits to be obtained from such studies provide significant information concerning the interactions among various color centers, impurities, and the lattice.

These topics are investigated by the methods of electron paramagnetic resonance (EPR) and optical absorption spectroscopy at controlled temperatures between $4.20 \mathrm{~K}$ and $300 \% \mathrm{~K}$.

Chapter II of this thesis gives a brief survey of the known color centers in the aikali halides, including currently accepted models for the color centers, optical characteristics of alkali halide crystals containing color centers, and the role of EPR in the color center research. Also included is a brief discussion of the principles of EPR. The experimental methods are described in Chapter III. Included in this section is a description of an EPR spectrometer, an optical absorption spectrometer, and a liquid helium cryostat for combined EPR and optical studies.

Chapter IV presents the results of EPR studies of the $x$-ray conversion of $\mathrm{Fe}^{2+}$ in $\mathrm{NaF}$ to $\mathrm{Fe}^{3+}$ and $\mathrm{Fe}^{+}$, and the interaction of $\mathrm{Fe}^{3+}$ (an S-state ion) with the host lattice. These EPR results 
prepare the way for studies of the optical processes in $x$-1rradiated NaF samples with and without the Iron impurity. The results of these optical studies are presented in Chapter V. Finally, the observation of a previousiy unobserved unstable optical absorption band in NaF is discussed in Chapter VI.

Two appendices are given for reference purposes in the present thesis; one of them is concerned with the spin Hamiltonian for an S-state ion such as $\mathrm{Fe}^{3+}$ in a cubic crystalline field, and the other is concerned with the nuclear hyperfine interaction. 
CHAPTER II

BASIC CONCEPTS

Color Centers

Color centers in the alkali halides have been a research topic for over fifty years. Recently many excellent review articles with extensive bibliographies have appeared.1-5. Therefore, in the brief summary of relevant color center topics which follows, only very recent work will be cited in the list of references. Details of the other topics can be found in the review articles.

The color center which has received the most attention is the F center. The F center is simply an electron trapped at an anion vacancy in the lattice (see Fig, 1). It has the simplest structure of all the color centers in the alkali halldes and is responsible for the most prominent (optical absorption) band in "colored" crystals. This band, which is very broad, ${ }^{+}$is called the F-band since it is due to optical transitions of the F center electron. A simple theory of the $F$ center treats the electron as being in a coulomb potential well, and the problem is similar to the hydrogen atom problem. The ground state is a ls orbital state and the first excited state is a triply degenerate $2 \mathrm{p}$ state which lies just below the conduction band of the solid. The interaction of the electron with the lattice is best visualized by use of a configuration coordinate diagram as shown in Fig。2(a)。This figure illustrates a plot of energy vs。

* The term "colored" crystal is used throughout this thesis to indicate any crystal which contains any type of color center.

+ in NaF the F-band is centered at $\lambda=340 \mathrm{~m} \mu$ and it has a width of about $.51 \mathrm{ev}$. See Ref。 2 for a complete listing of color centers in all the alkali halides. 

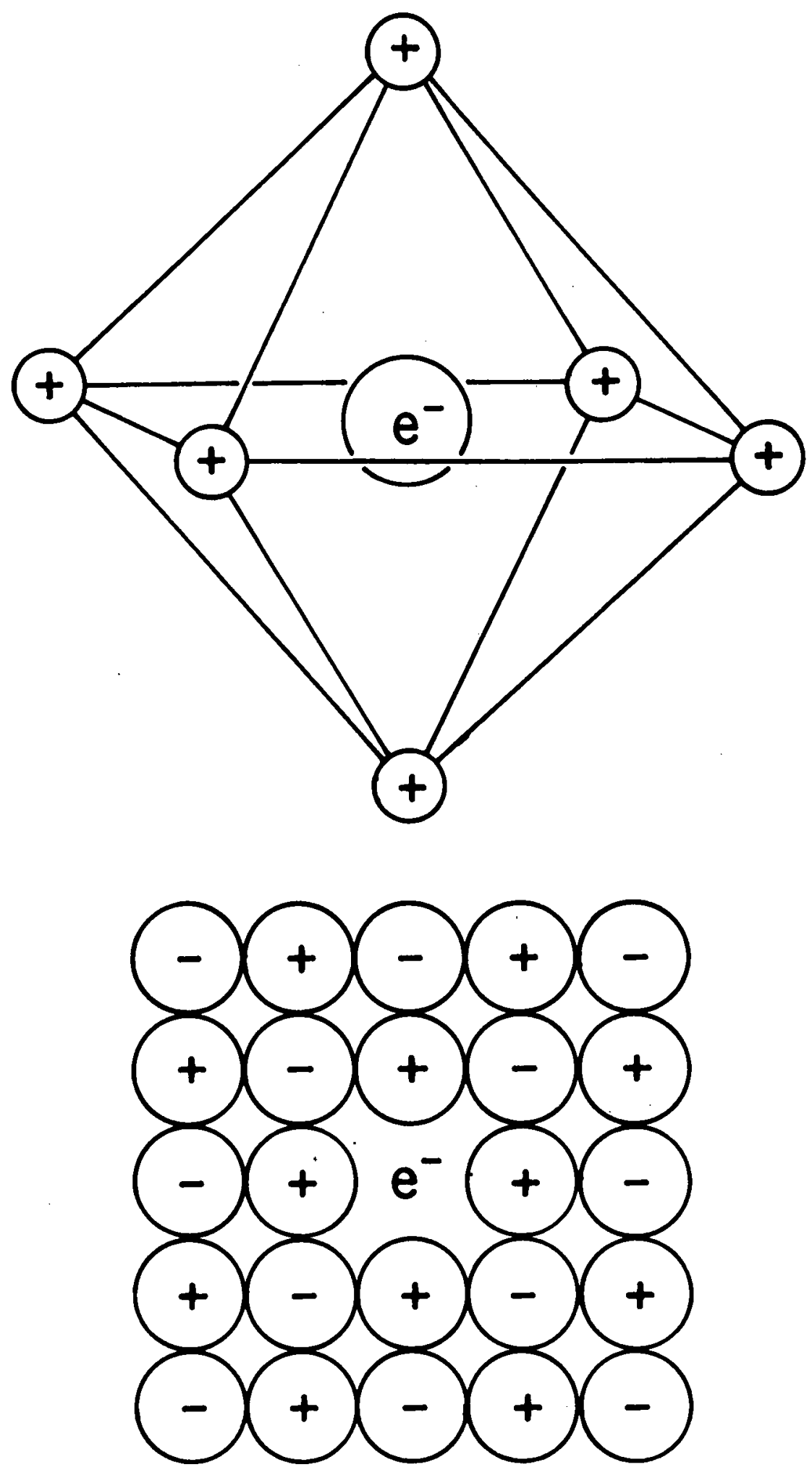

Fig. 1. Schematic diapram of an F center: Sterescopic view plus a section in the (100) plane. 
a configuration coordinate for the ground and first excited states of the $F$ center. The coordinate $q$ describes the "breathing" mode, which Is the principal vibrational mode of the ions in the neighborhood of the point defect. This vibrational mode is 11lustrated in Fig. 2(b), where the six nearest neighbors of the $F$ center move in unison as shown. A configuration diagram such as the one shown in Fig. 2(a) can be used to explain the following experimentally observed properties of F centers: (1) why the F-band is not a sharp line such as observed for the absorption spectrum of free atoms; (2) why the observed luminescence of the $F$ center has a large Stokes shift*; (3) why it is possible to have radiationless transitions; and (4) why optical bleachIng is accompanied by photoconductivity. To see why the above effects are reasonable, consider the absorption process in terms of the diagram in Fig. 2. During an optical transition, the nuclear coordinates, and hence $q$, remain fixed according to the Frank-Condon princtple. 6 The ground state and" the first exclted" state, however, have many vibratlonal levels which are lilustrated by a set of parallel horizontal Ines above the points $A$ and $G$, respectively, in Fig. 2 . Hence, the values of the nuclear coordinates at any given time depend on the vibrational state of the neighboring 1ons. Typically, the center of the absorption band might correspond to a transition from point A to point $B$ on the configuration coordinate dlagram. In this case the absorbed phoțon has an energy $h \nu_{A B}=E_{B}-E_{A}$. At room temperature,

* A Stokes shift is a shift in frequency of the Iuminescent ilght $\left(v_{l}\right)$ from the exciting 11ght. $\left(v_{e}\right)$. In returning to the ground state the electron emits a photon and it also can give energy to the lattice; hence, $v_{\ell}<v_{\ell}$. 
(a)

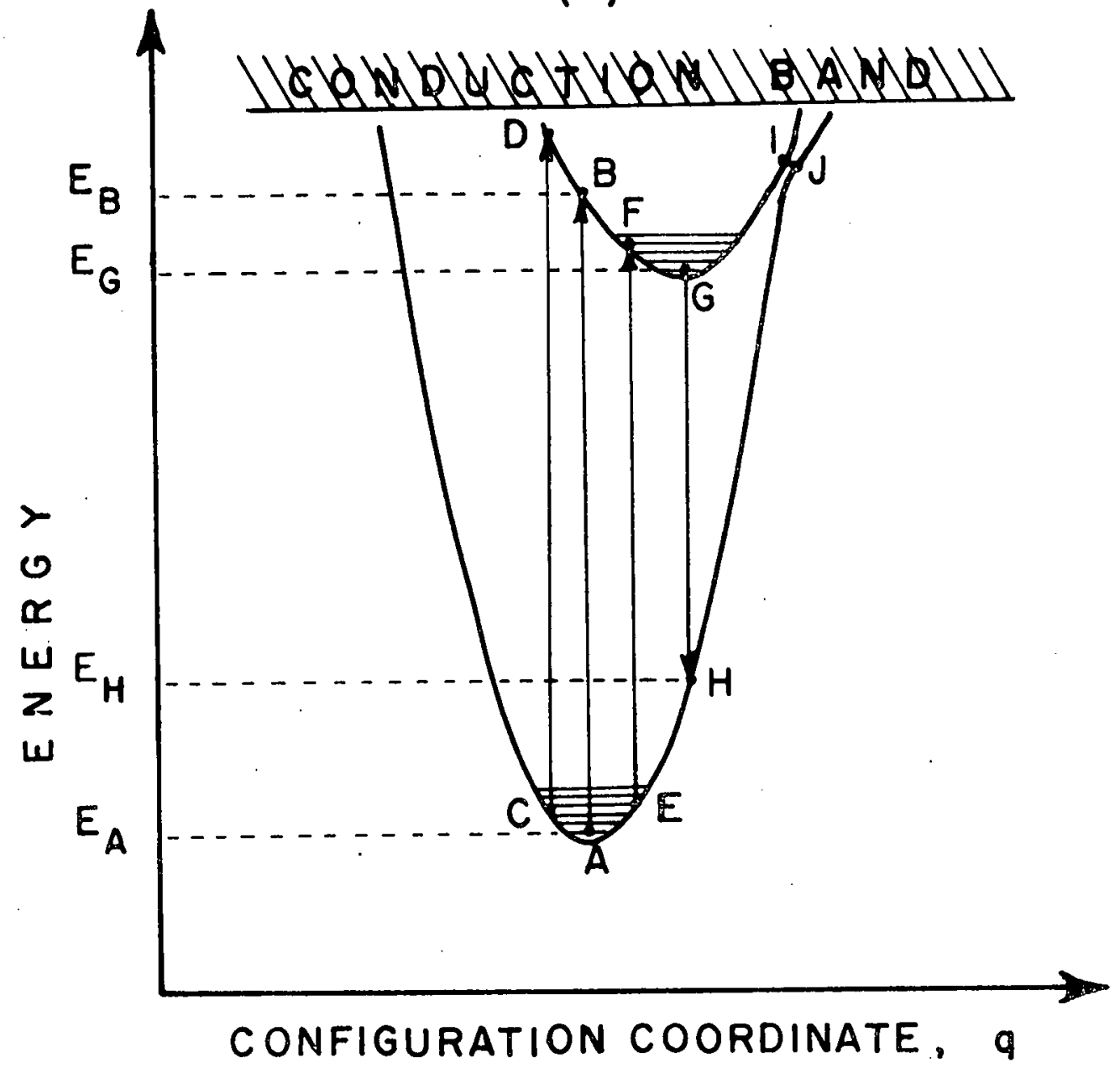

(b)

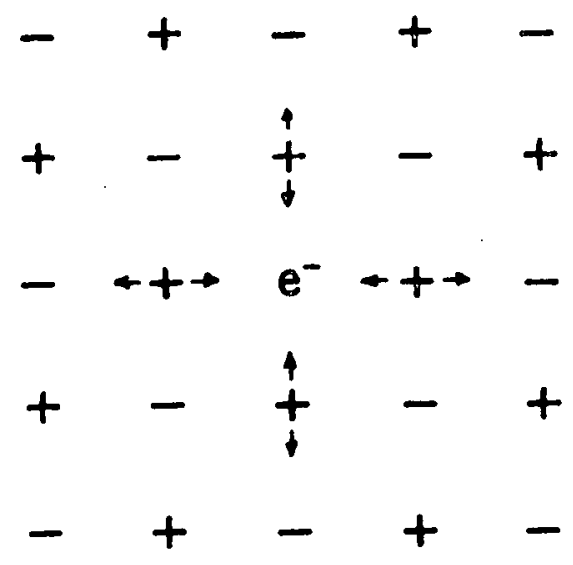

Fig. 2. (a) Confiruration coordinate diagram of an $F$ center. (b) Breathins mode of vibration of the six nearest neighbor cations of the $F$ center. 
many of the higher energy vibrational states will be excited and transition from $C$ to $D$ and from $E$ to $F$ will also occur, giving rise to the width of the absorption band.

When the electron is in the excited state, it has a wave function different from the ground state wave function. The energy of the excited electron will be a different function of $q$ and, in general, $q$ will have a new equilibrium value. In returning to the ground state, the electron may give up a part of its energy to the lattice and "drop" to point $G$ in the configuration coordinate diagram. From point $G$ it can spontaneously drop back to the ground state at point $\mathrm{H}$ emitting a photon $h v_{G H}<h v_{A B}$ " The energy of the emitted photon is observed as luminescence with a large stokes shift corresponding to the reduction of frequency. If the thermal energy is sufficiently large, the electron at point $G$ can go to point $I$ and to point " $J$ and then relax" to point $A$ giving up ail its energy to the lattice in a radiationless transition. There is also the possibility that the excited $F$ center electron will absorb sufficient thermal energy and be excited into the conduction band, contributing to the photo-conductivity. Once in the conduction band, it is free to migrate until it becomes trapped at another defect or impurity center。

If ilght in the region of the F-band (simpiy F-light) is incident on a colored crystal containing primarily $F$ centers, then the (absorption) Intensity of the F-band decreases and new bands at longer wavelengths are known to develop. These new bands are assoclated with new color centers called F-aggregate centers, wh1ch are nearestneighbor combinations of $F$ centers. The simplest of the F-aggregate centers is the $\dot{M}$ center, which is a pair of $F$ centers at nearest 
neighbor sites as shown in Fig。3(a). The M-band is the first of the optical absorption bands on the long wavelength side of the F-band to appear during optical bleaching。 After the M-band starts to grow, two bands called $R_{1}$ and $R_{2}$ grow with constant intensity ratio. These bands are due to two different transitions of the $R$ center which is 1llustrated in Fig。3(b)。As shown, the $R$ center is a nearest neighbor combination of three $\mathrm{F}$ centers. Finaliy, the $\mathrm{N}_{1}$-and $\mathrm{N}_{2}$ bands appears. These bands are attributed to two different $\mathrm{N}$ centers, called $\mathrm{N}_{1}$ and $\mathrm{N}_{2}$ centers. Both $\mathrm{N}$ centers are a combination of four $\mathrm{F}$ centers (see Fig。 $3(c) ; N_{1}$ has a planar configuration and $N_{2}$ is a tetrahedron。 Recent work on F-aggregate centers is summarized in Ref。2. Of the Faggregate centers, the $M$ center has recelved the most attention and its model is very well established。 7,8 The $N$ centers have recelved comparatively little attention and there is still doubt concerning the proposed models. 9

Other color centers play an important role in the $F \rightarrow F$-aggregate conversion. These are electrically charged color centers, such as the $\mathrm{F}^{\prime}$ and $\mathrm{F}_{2}{ }^{+}$centers. A prime (') indicates that the otherwise neutral color center has an excess electron. Hence an $F^{\prime}$ center is an $F$ center with two electrons instead of one. A superscript plus (t) indicates the lack of an electron. Hence, and $M^{+}$center is an $M$ center with only one electron. The accepted model of the $M$ center is often called the $F_{2}$ model of the $M$ center, $F_{2}$ meaning a nearest neighbor combination of two $F$ centers. Similarly, the currently accepted model of the $R$ center is the $F_{3}$ model。

It has been known for a long time that impurities affect color center processes, 10 in particular, the colorability of crystals. 
(a)
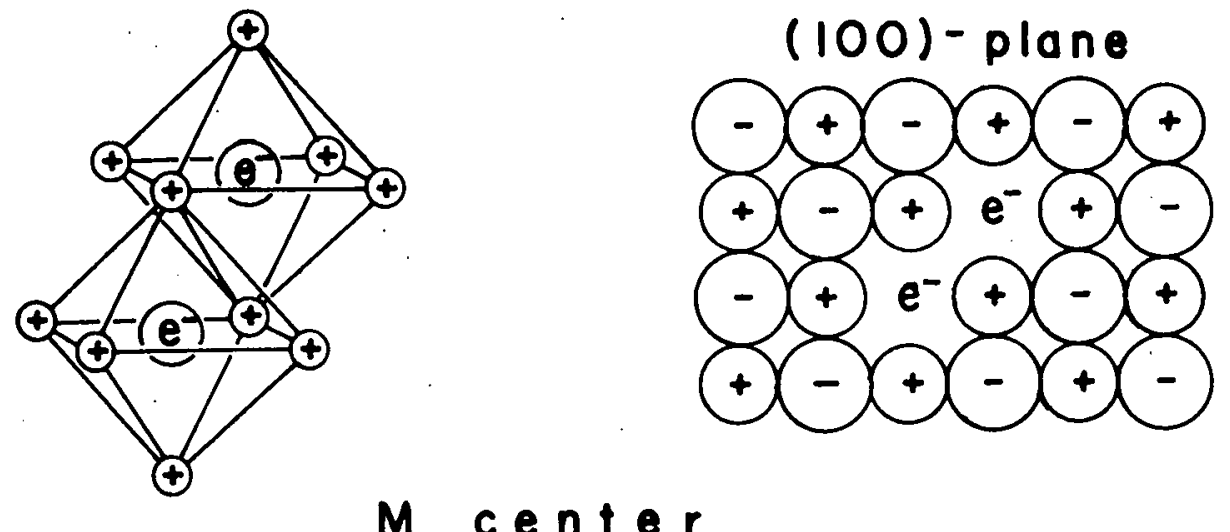

Mcenter

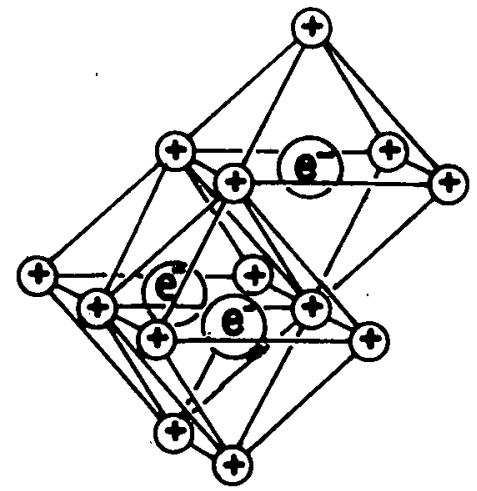

(b)
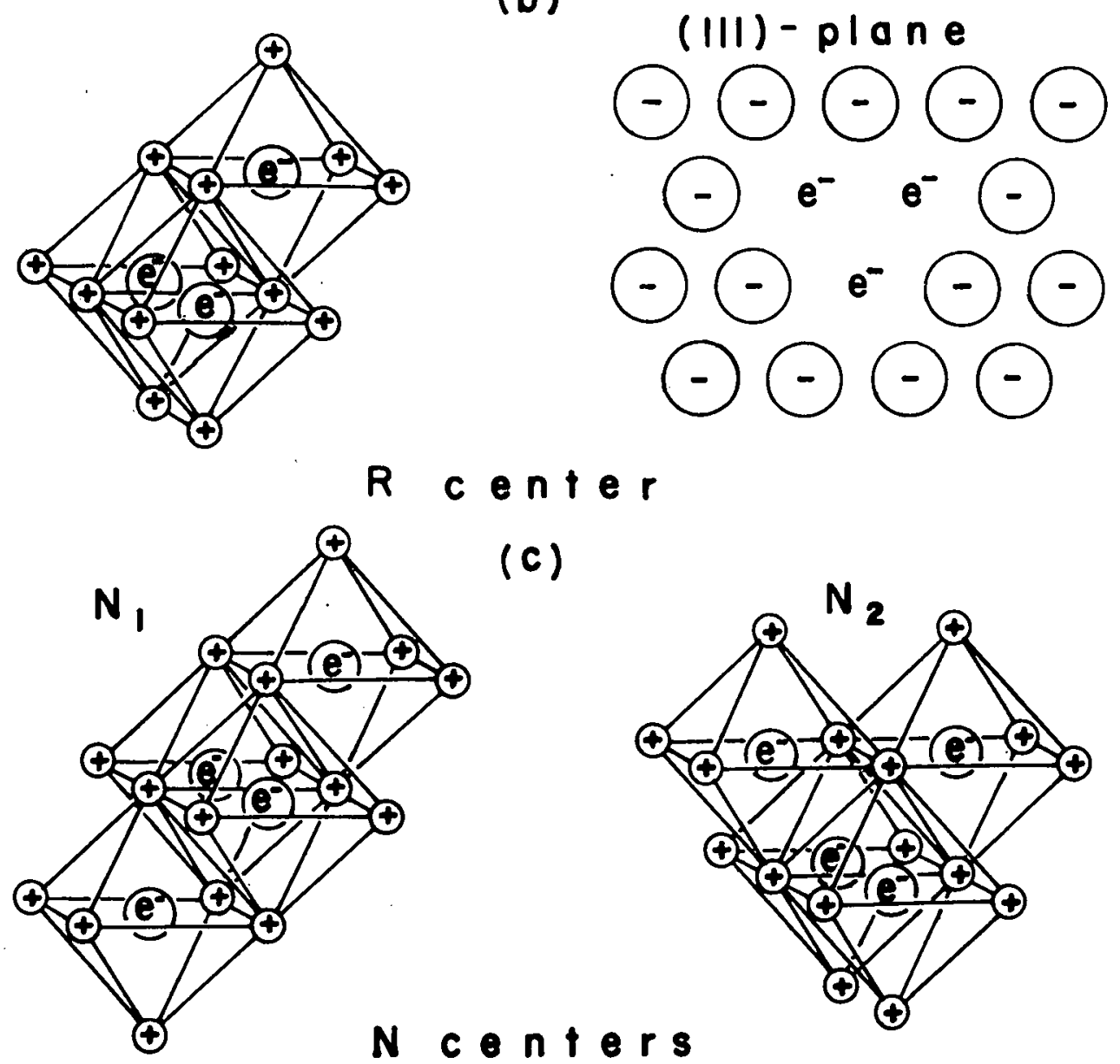

Fig. 3. Schematic diagrams of the current models for F-aggregate centers: (a) A sterescopic view of the $M$ center and a section in the (100) plane, (b) A sterescopic view of the $R$ center and a section in the (111) plane, (c) Stereoscopic view of the $N_{1}$ and $N_{2}$ centers. 
Impurity ions may be effectively electron or hole traps, and thus have an Influence on the coloration process. Some impurities are foreign atoms entering the host lattice in interstitial positions and thus causing local strains and defects in the lattice. Others are divalent or trivalent foreign atoms, entering the lattice substitutionally. In either case, divalent or trivalent impurity ions force the formation of charge compensating vacancies in the lattice to maintain local electrical neutrality. These vacancies may be bound to an impurity ion to form a well defined complex, or they may be unbound and free to migrate at sufficiently high temperatures.

\section{Electron Paramagnetic Resonance (EPR)}

The fundamental concepts of EPR and its applications are discussed by many authors。11-15 It is the purpose of this sumary to indicate briefly what EPR is and what Information can be obtained about paramagnetic lons by using EPR techniques.

To illustrate the concept of EPR consider the case of a single free electron in a static magnetic field $\vec{H}$. The electron has a magnetic moment

$$
\vec{\mu}=-g B \vec{S}
$$

where

$$
\begin{aligned}
& g=\text { electronic } g \text { factor, } \\
& B=\text { Bohr magneton, } \\
& \vec{S}=\text { the electron's spin angular momentum }
\end{aligned}
$$

When placed in a magnetic field, the magnetic moment of the electron interacts with the fleld such that the interaction energy is given by 


$$
E=-\vec{H} \cdot \vec{H}=g B \vec{S} \cdot \vec{H} .
$$

This is what is commonly called the Zeeman interaction and it causes the electron to have two energy levels characterized by the spin quantum number $m_{s}= \pm 1 / 2$ as shown in Fig. 4. When $H=H_{0}$, the difference in energy between the two Zeeman levels is given by

$$
\Delta \mathrm{E}=\mathrm{gBH} \mathrm{H}_{\mathrm{O}}
$$

Classically the electronic magnetic moment $\vec{\mu}$ can be considered to precess about the magnetic fleld $\vec{H}_{0}^{11}$ The application of an oscillating magnetic field of frequency $v$, perpendicular to $H$, can cause magnetic dipole transitions between these two spin levels if the resonance condition, $h \nu=\Delta E$, where $h$ is Planck's constant, is satisfied. For $g$ values around two, magnetic fields of $10^{3}$ to $10^{4}$ gauss correspond to frequencles of about $10^{10} \mathrm{cps}$.

Consider now that this electron is placed in a solid substance. For convenience consider the case of an $F$ center in an otherwise perfect lattice. The $F$ center electron is no longer free and it interacts with its environment. The charged ions surrounding the $F$ center produce a crystalline field which will play a major role in determining the $F$ center wave function. All the nelghboring nuclei in the lattice have magnetic moments. These nuclear magnetic moments will interact with the magnetic moment of the $F$ center electron (hyperfine interaction). Detalls of the hyperfine interaction are discussed in Appendix I. The resonance condition is altered by the hyperfine interaction. When a single nucleus of spin $I$ is involved, the resonance condition is given by (See Eq. A-21): 


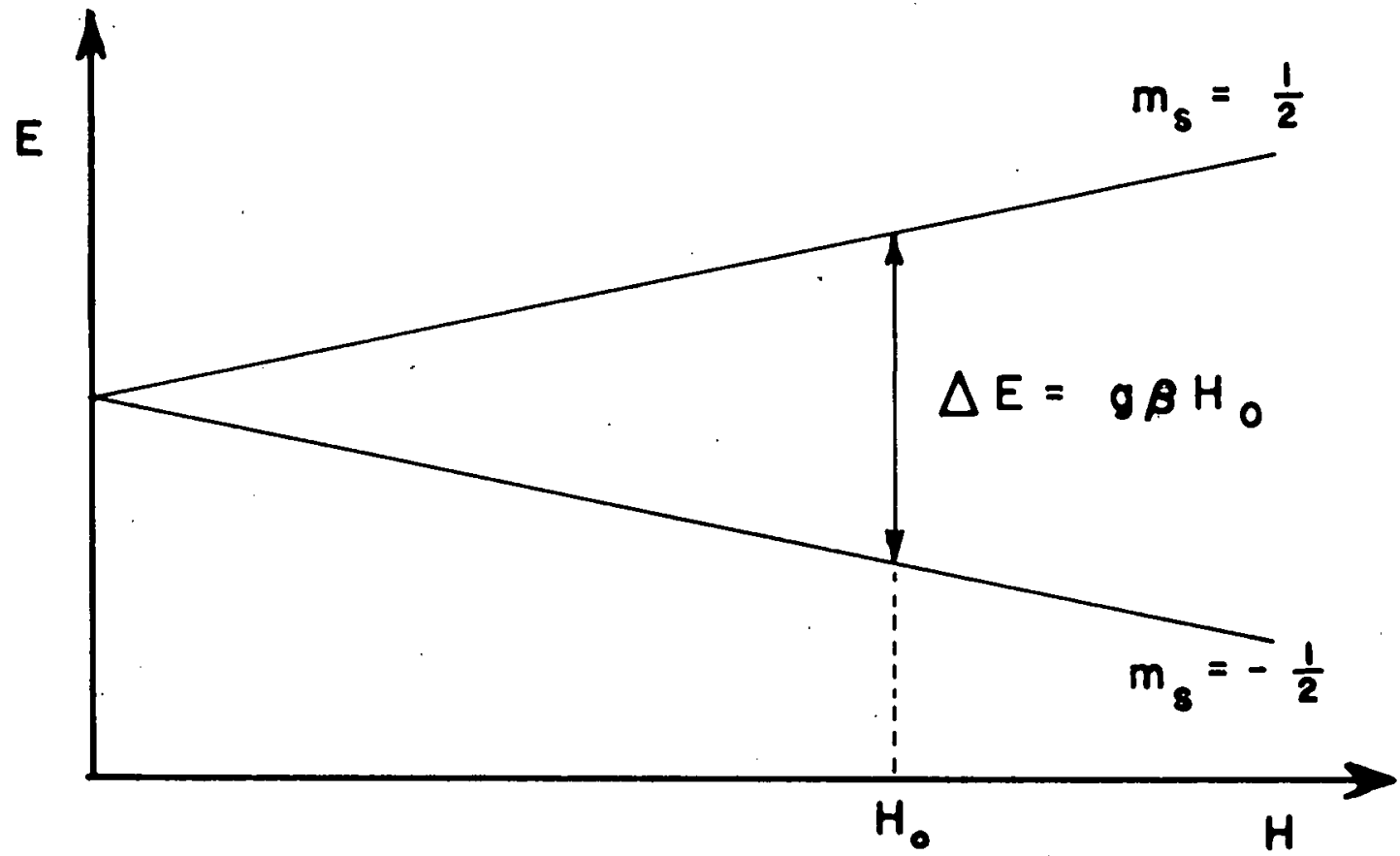

Fig. 4. Energy level diagram for an electron in a magnetic field. 


$$
h v=g B H+M_{I} W_{H F S}
$$

where

and

$$
\begin{aligned}
\mathrm{W}_{\mathrm{HFS}}= & \delta+\gamma\left(3 \cos ^{2} \theta-1\right) \ldots \\
\mathrm{M}_{\mathrm{I}}= & \text { magnetic quantum number of the } \\
& \text { interacting nucleus with spin } \mathrm{I}=\mathrm{I}, \\
& \mathrm{I}-1, \ldots \ldots(\mathrm{I}-1),-\mathrm{I} ;
\end{aligned}
$$

$\theta=$ the angle between $\vec{H}$ and the direction of the radius vector from the center of the $F$ center to the nuclear site.

The value of $g$ in Eq. $\dddot{4}$ is in general different from the free electron $g$ value of 2.0023. Also in more general cases the value of $g$ can depend on the direction of $\mathrm{H}$ relative to a crystal and in this case the $g$ value is described by a tensor. This topic is treated in detail In Ref。15. In most EPR experiments $V$ is kept constant and $H$ is varied。 Thus, transitions now occur when

$$
H=\frac{1}{g \beta}\left(h v-M_{I} W_{H F S}\right)=H_{0}-M_{I}\left(\frac{W_{H F S}}{g \beta}\right) \text {, }
$$

Instead of $\mathrm{H}_{0}$. Hence, where without the hyperfine interaction there would be one resonance line at $\mathrm{H}=\mathrm{H}_{0}$, with the hyperfine interaction there are $2 I+1$ hyperfine IInes symmetrically distributed on both sides of $\mathrm{H}_{\mathrm{O}}$. Details of the EPR spectra due to $\mathrm{F}$ centers in alkali halides have been discussed by many authors。 $3,14,15$

Any 1 ion or system of electrons with $\overrightarrow{\mathrm{L}}$ or $\overrightarrow{\mathrm{S}} \neq 0$ is paramagnetic 15 Hence, some color centers other than $F$ centers are paramagnetic。 EPR signals have been observed for $R$ centers 16,17 ( 3 eilectrons with $S=1 / 2,3 / 2$ ) and the metastable triplet state of $M$ centers 
(2 electrons with $S=1)$ Also, transition element lons have an unfilled inner electron shell and have $\vec{s}$ and/or $\vec{L} \neq 0$, making them paramagnetic. Like the $F$ center electron, when a paramagnetic ion is placed in a solid substance, it interacts with its environment. If there are neighboring nuclear magnetic moments, there is a hyperfine interaction just as in the $\mathrm{F}$ center case. In general, besides the hyperfine interaction, there are the following: (1) the spinorbit interaction, (2) the crystalline field interaction, and (3) a spin-spin interaction between electrons.

The Hamiltonian which describes the paramagnetic Ion in a solid substance takes into account all of the interactions of the ion with its environment and is quite complicated. To explain an EPR spectrum, it is not necessary to work with this "complete" Hamiltonian, If . This is because EPR involves only magnetic dipole transitions which are described by a change of the magnetic quantum numbers only。 Hence, it is convenient to work with a Hamiltonian which has only spin operators. This Hamiltonian is called a "spin Hamiltonian"18 and is labeled I $_{\mathrm{S}}$. $_{\mathrm{S}}$ is obtained from \# by calculating

$$
\dddot{H}_{S}=\int \psi^{*} \sharp \psi d \tau
$$

where $\psi$ is the orbital wave function of the paramagnetic ion and the integral is over the orbital variables only. These integrals then appear as constants (or parameters from the spectroscopists' point of view) in It $S^{\circ}$

An example of a paramagnetic ion is $\mathrm{Fe}^{3+}$. This ion has a ${ }^{6} \mathrm{~S}_{5 / 2}$ ground state. When placed into an alkali halide, $\mathrm{Fe}^{3+}$ must 
have a spin Hamiltonian which takes into account three effects: the Zeeman interaction of a magnetic moment due to $\ddot{S}=5 / 2$ with the external static magnetic field $\mathrm{H}$, (2) the hyperfine interaction of the magnetic moment with all the neighboring nuclear magnetic moments, and (3) the effects of the crystalline field and spin-orbit coupling, which are effects that are greater than first order for the case of an S state ion. The latter interactions (3) give rise to what is called fine structure and details of this term in the spin Hamiltonian are presented in Appendix II.

By examining an EPR signal due to a paramagnetic ion in detail, one can determine what types of interactions are taking place. Knowing this, it is possible to "fit" the observed spectra with an appropriate spin Hamiltonian with the right values of the spin Hamiltonian parameters. At this point one is able to infer: (1) what the spin of the paramagnetic ion is, (2) whether or not it is an S-state ion, (3) the spin quantum numbers of the nuclel with which it interacts, (4) the hyperfine coupling constants of the interacting nuclei (See Appendix I), and (5) where in the lattice the paramagnetic ion is located. Many other things can be inferred 3,15 but most important is the fact that EPR gives the experimentalist a microscopic view of the environment of a paramagnetic ion. 
CHAPTER III

EXPERIMENTAL SET-UP AND TECHNIQUES

As mentioned previously, color center research involves the use of both an optical spectrophotometer for measuring the optical absorption bands due to color centers and an EPR spectrometer for investigating paramagnetic impurities and color centers. These two instruments along with a versatile cryostat for combined EPR and optical work will be described separately in the following sections.

\section{Optical Spectrophotometer}

Color centers manifest themselves macroscopicly by absorbing light in characteristic bands lying in the visible, near infrazed, or near ultraviolet portions of the optical spectrum. The measured optical absorption is characterized in terms of an absorption coefficient of the material, $\alpha(\lambda)$, where $\lambda$ is the wavelength of the incident 1ight。Consider the situation 1llustrated in Fig。 5 where a monochromatic beam of light of wavelength $\lambda$ and intensity $I_{0}$ is incident normally on a crystal of thickness $d$. The transmitted beam has an Intensity $I$ where $I<I_{0}$ (assume that there is no diffuse scattering of light)。 At a point $x$ in the crystal the intensity is $I(x)$. In traversing a distance $d x$ in the crystal, the intensity of the beam is diminished by an amount $d I=-\alpha(\lambda) \cdot I(x) d x 。$ By integrating this differential equation with the proper boundary conditions, the following solution is obtained:

$$
I(x)=I_{0} \exp [-\alpha(\lambda) x]
$$

Hence, the intensity of the transmitted light is obtained as 


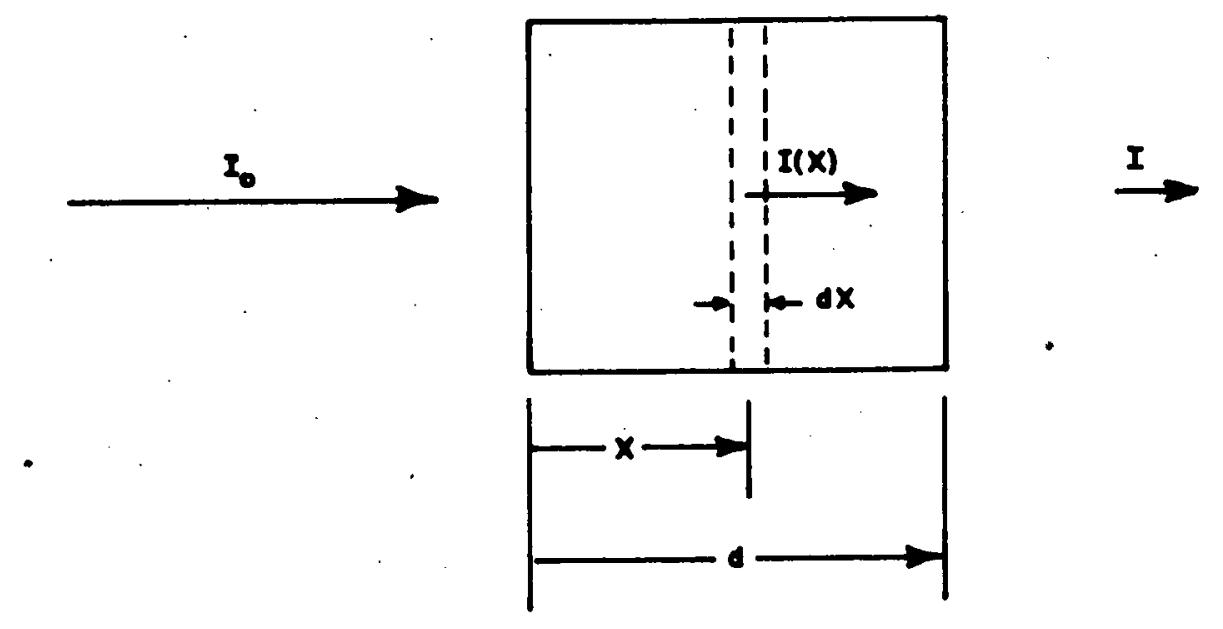

Fig. 5. Diagram 1llustrating the geometry of a sample in a spectrophotometer light beam. 


$$
I=I(d)=I_{0} \exp [-\alpha(\lambda) d]
$$

An optical spectrophotometer measures ad as a function of $\lambda$ for a given crystal. ad is called the optical density. The spectrophotometer that was used in the present experiments is a Bausch and Lomb 505 recording spectrophotometer. Details of this instrument are given in a Bausch and Lomb manual.19 Therefore, only a brief explaination of how the instrument measures optical density, $\alpha(\lambda) d$, w111 be presented here。 A schematic diagram of the spectrophotometer is given in Fig. 6. A condensing lens system (B) collects light from a source of light (A) and focuses it on the entrance slit (C) of a monochrometer. The light source (A) is a tungste: lamp for the visible spectrum $(\lambda=400 \mathrm{~m} \mu \rightarrow 700 \mathrm{~m} \mu)$ and a deuterium discharge lamp for the near ultra-violet spectrum $(\lambda=200 \mathrm{~m} \mu \rightarrow 400 \mathrm{~m} \mu)$. A monochromatic beam of wavelength $\lambda$ emerges from the exit slit and two mechanically connected chopping wheels chop these beams at (E) In such a way that they are alternately transmitted as shown in Fig. 6. Each beam is chopped at $60 \mathrm{cps}$ such that it is on once each cycle for a period of $1 / 4$ of a cycle. One beam passes through the sample (F) under investigation and is called the sample beam, while the other is used as a reference and is called the reference beam. The reference and sample beams are focused at $(G)$ onto the photomultiplier cathode. The reference beam and the sample beam are alternately incldent on the photomultiplier (H). The photomultiplier output is proportional to the incident light intensity and it is amplified by a preamplifier (I) as shown. The sensitivity of the photomultiplier is determined by the high voltage applied to its dynodes. This voltage is changed automatically to maintain the proper sensitivity, as will 


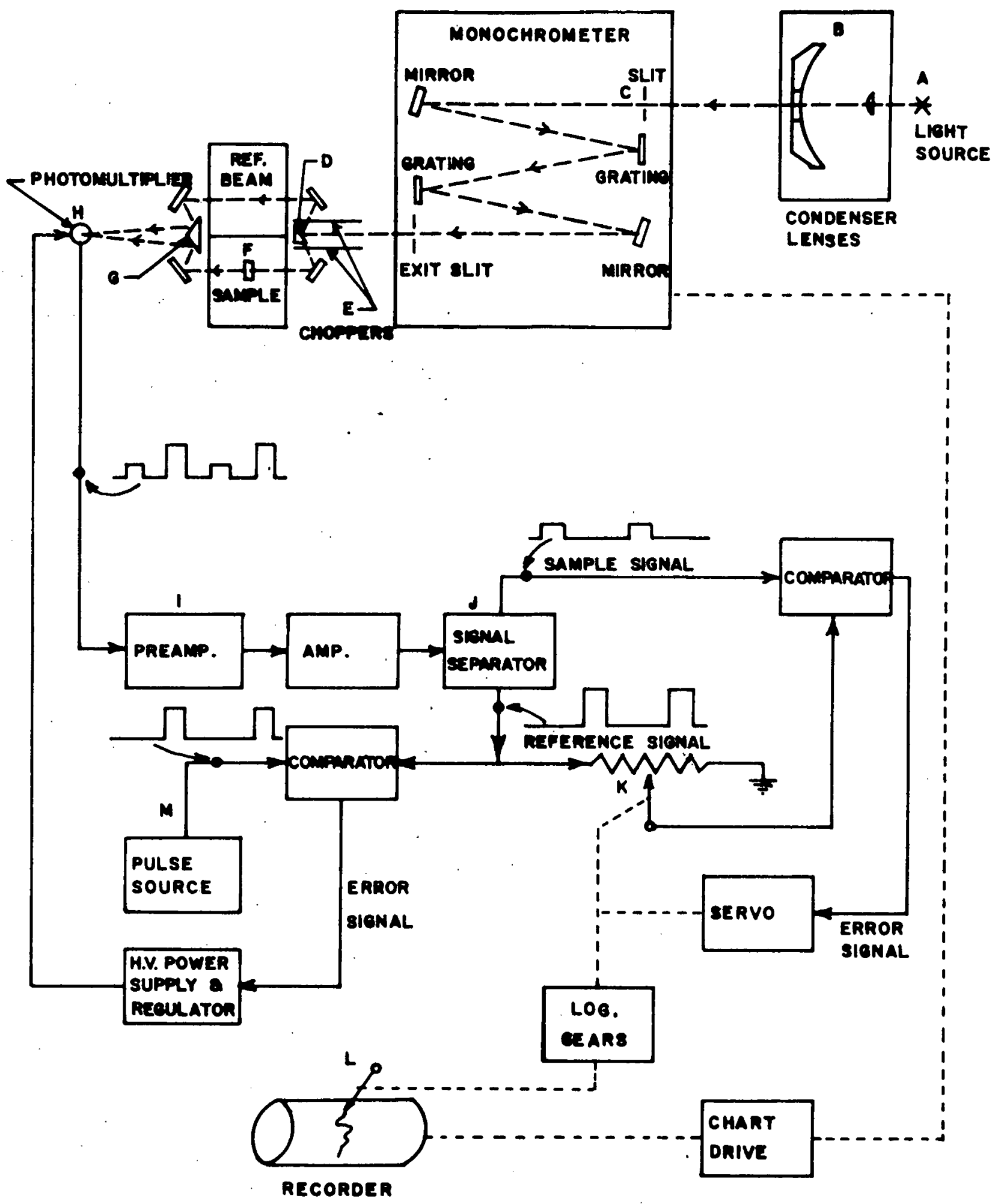

Fig. 6. Schematic diagram of an optical absorption spectrophotometer (a Bausch and Lomb Spectronic 505). 
be described below. After being amplified, the reference and sample signals are separated at $(\mathrm{J})$. The reference signal is applied to a potentiometer $(\mathrm{K})$ whose slider is mechanically connected to a recorder pen $(L)$. By means of a servo mechanism, the potentiometer slider is constantly maintained at a position such that the voltage at the slider is' equal to the voltage of the sample signal. Hence, the ratio of the distance of the potentiometer slider from the grounded end of the potentiometer to the length of the potentiometer is equal to (I/I) However, the mechanical connection between the potentiometer and the recorder pen is via logarithmic gears. Thus, the pen records a signal proportional to $\left|\ln \left(I / I_{0}\right)\right|=\alpha(\lambda) d_{0}$. Now the absorption coefficient is proportional to the concentration of color centers absorbirg light of wavelength $\lambda_{0}$ Therefore, at a given wavelength, the recorded output is proportional to the concentration of absorbing color centers. A continuous curve $(\alpha(\lambda) d$ vs。 $\lambda)$ is obtained by scanning the monochromerer over a range of values of $\lambda_{0}$

The dual-beam feature ( sample beam and reference beam) prevents changes in $I_{0}(\lambda)$ with wavelength from being interpreted as sample absorprion. It also reduces noise due to fluctuations in the intensity of the 11ght source. As part of this: feature, the reference signal is maintained at a constant level by comparing the reference signal to the output of a constant voltage pulse source $(M)_{0}$ and by changing the high voltage applied to the photomwliplier. This is done electronlcally by using the difference in voltage between the reference signal and the pulse source as an error signal for the high voltage regulator. If the reference signal decreases, the high voltage is increased. This 
increase of high voltage on the photomultiplier provides a larger accelerating potential for the electrons freed from the photocathode. Hence, the sensitivity of the photomultiplier is effectively increased and the output current is maintained at a constant level when the reference beam is incident on the photomultiplier.

For an optical bleaching experiment, it is necessary to shine a bleaching light of a selected wavelength on the sample, and at regular intervals measure the changes in the optical absorption bands. For this purpose, the sample compartment shown in Fig。 6 is modified as 1llustrated in Fig. 7. The sample is mounted in a sample holder which can be rotated by $90^{\circ}$. In the $0^{\circ}$ position the sample is in the sample beam of the spectrophotometer and in the $90^{\circ}$ position, it is in the bleaching beam. The bleaching beam is produced by means of a 150 watt high pressure mercury arc lamp $(N)$ and a monochrometer (0). This beam is perpendicular to the direction of the sample beam and $1 t$ is focused on the sample by means of a lens (P)。 The intensity of the sample beam is much less than the intensity of the bleaching beam。 Hence, bleaching caused by the sample beam during an optical density measurement is negligible.

\section{EPR Spectrometer}

The spectrometer assembly used in the present work consists mainly of (1) a Varian Model V4500 EPR spectrometer, operated in the $X$ band of microwave frequencies ( 8.2 to $12.4 \mathrm{kMcps}$ ) with magnetic field modulation at $100 \mathrm{kcps}$ and audio frequencles, and (2) an associated Varian model V3603 12 inch rotating laboratory magnet。 A block diagram of the assembly is given in Fig。 8. Since the 


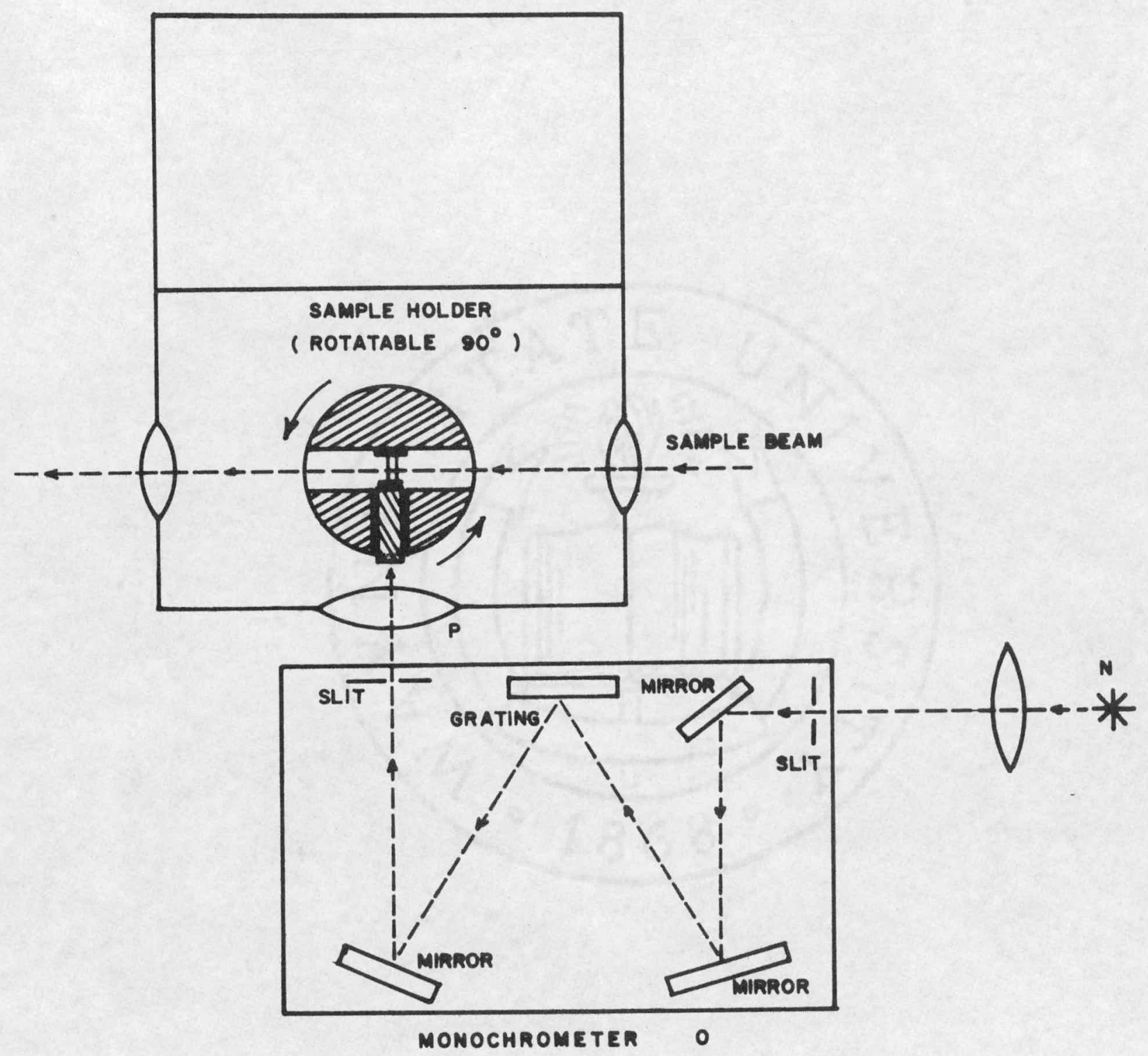

Fig. 7. Modification of the spectrophotometer sample compartment for optical bleaching experiments. 


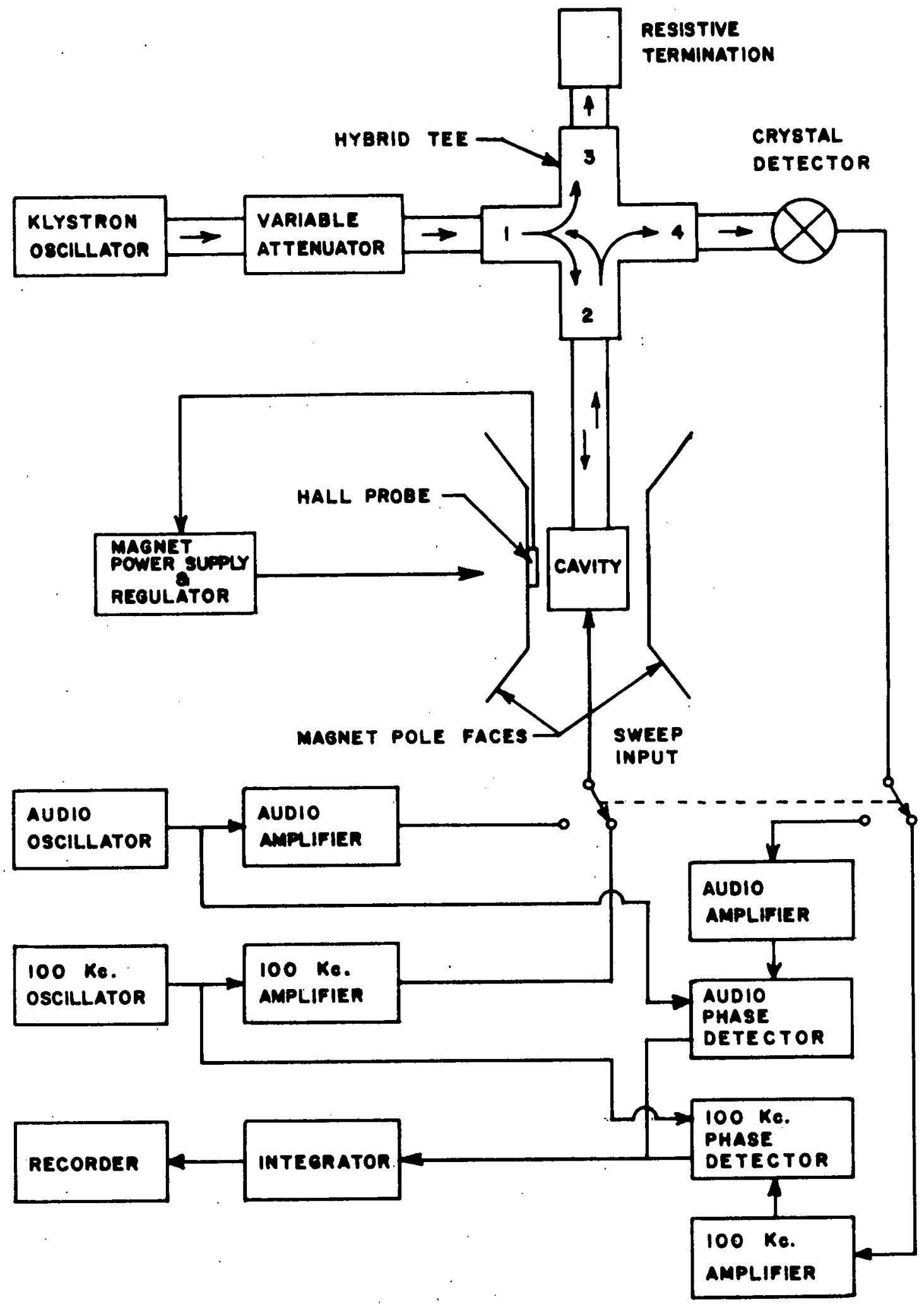

F1g. 8. Schematic diagram of an EPR spectrometer. 
operational details of this system are given in a Varian manual, 20 only a brief summary of its operation will be given here.

The sample which contains paramagnetic ions must be placed in an environment where the resonance condition $h \nu=\mathrm{gBH}_{0}$, can be satisfled. As mentioned in Chapter II, two things are necessary to satisfy the resonance condition: (1) a static magnetic field $\mathrm{H}_{0}$, and (2) an oscillating magnetic field of frequency $\nu$ perpendicular to $\mathrm{H}_{\mathrm{O}^{\circ}}$ The static field is supplied by the large 12 inch electromagnet. The sample is located in a microwave cavity which is centered in the alr gap of this electromagnet. The purpose of this microwave cavity is to supply the oscillating magnetic field。 The sample is positioned in the cavity in the region of maximum oscillating microwave magnetic fleld. This oscillating field may be thought of in terms of two rotating magnetic fields: one rotating in the same sense as the precessing magnetic moments of the paramagnetic Ions in the sample and the other rotating in the opposite sense. The microwave field which rotates in the same sense as the precessing magnetic moments provides the required pertubation to cause magnetic dipole transitions in the sample. The microwave frequency $v$ of the oscillating field is kept fixed at about $9.3 \mathrm{kMc}$ and the static magnetic field is varied until the resonance condition is satisfied.

The spectrometer must detect the absorption of microwave power by the sample when the resonance condition is satisfied. To see how this is accomplished, we must first digress and explain how the microwave radiation reaches the cavity。 Referring to $F i g$, 8, it can be seen that the microwave power from the klystron is supplied to arm one of the hybrid tee. This power is then divided between a 
resistive load in arm three, and arm two. Since the impedance of the microwave cavity with the sample in place is matched to the impedance of arm two when the resonance condition is not satisfied, all the power in arm two is absorbed by the microwave cavity. When the resonance occurs, the sample absorbs power and, by so dolng, changes the Impedance match between the cavity and arm two of the hybrid tee. This mismatch is proportional to the power absorbed by the sample, and causes a reflected wave to propagate back from the cavity to arm two, where it is divided between arm four and arm one. Arm four is terminated by a crystal detector. This detector provides a voltage proportional to the microwave power incident on $1 t$, which in turn is proportional to the mismatch of the cavity. Hence, by monitoring the voltage output of the crystal detector while the static magnetic field Is swept, one can detect the signal representing the power absorption, which will be referred to: as the EPR signal of the sample in the following. The situation is slightly complicated by the fact that when the sample absorbs power in the microwave cavity, it also shifts the resonant frequency of the microwave cavity. This shift in the resonant frequency from its off resonance value is known as dispersion. The spectrometer system as shown in Fig. 8 discriminates against dispersion and detects a signal proportional only to the absorbed microwave power by locking the klystron frequency to the microwave cavity. Detalls of this automatic frequency control (AFC) can be found elsewhere. 21

The signal observed at the crystal detector is a D.C. signal and it is accompanied by nolse which might obscure a weak EPR signal. In order to overcome this problem, the spectrometer system uses a 
combination of magnetic field modulations and narrow band detection. The magnetic field modulation is produced by a pair of Helmholtz sweep coils on opposite sides of the cavity. These colls are driven by a combination of a $100 \mathrm{kc}$ oscillator (or an audio frequency) and a power sweep amplifier. The AC magnetic field produced by these coils is parallel to the static magnetic field. This field modulation causes the sample to see a varying magnetic field which in turn modulates the signal seen by the crystal detector when the resonance condition is satisfied (see Fig。9)。' Without field modulation a DC signal appears at the crystal detector. This DC signal is proportional to the "bell shaped" resonance line obtained when $\mathrm{H}$ is scanned through the resonance point as shown in Fig。9. The AC signal due to the field modulation is amplified by a narrow band amplifier tuned to the field modulation frequency。 This "narrow band detection" eliminates all the noise except the small fraction present at the fleld modulation frequency.

The size of the amplified AC signal is proportional to the slope of the absorption line at that particular value of magnetic field. Further, the phase of the amplified signal shifts by $180^{\circ}$ when the slope of the absorption curve changes sign. Therefore, the amplified signal is sent through a phase sensitive detector, which uses the field modulation oscillator as a reference, and the result is a demodulated signal proportional to the first derivative of the absorption line as shown in Fig。9. To further reduce the noise content of this derivative signal it is passed through an integration network and finally it is displayed by a graphic recorder as shown in Fig。 8。 The shape of the recorded absorption line in 

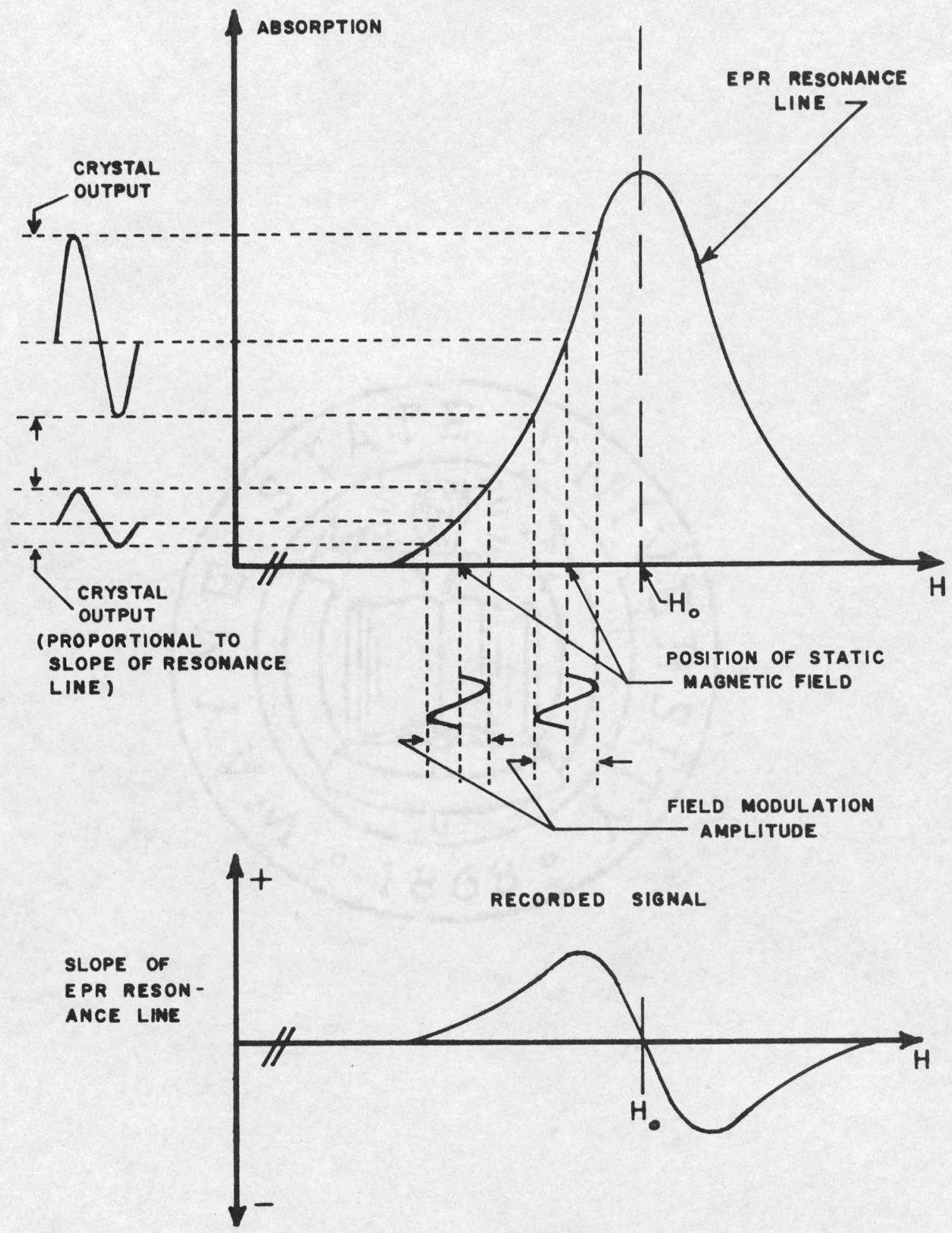

Fig. 9. Output of an EPR spectrometer illustrating why the recorded signal is the first derivative of the absorption line. 


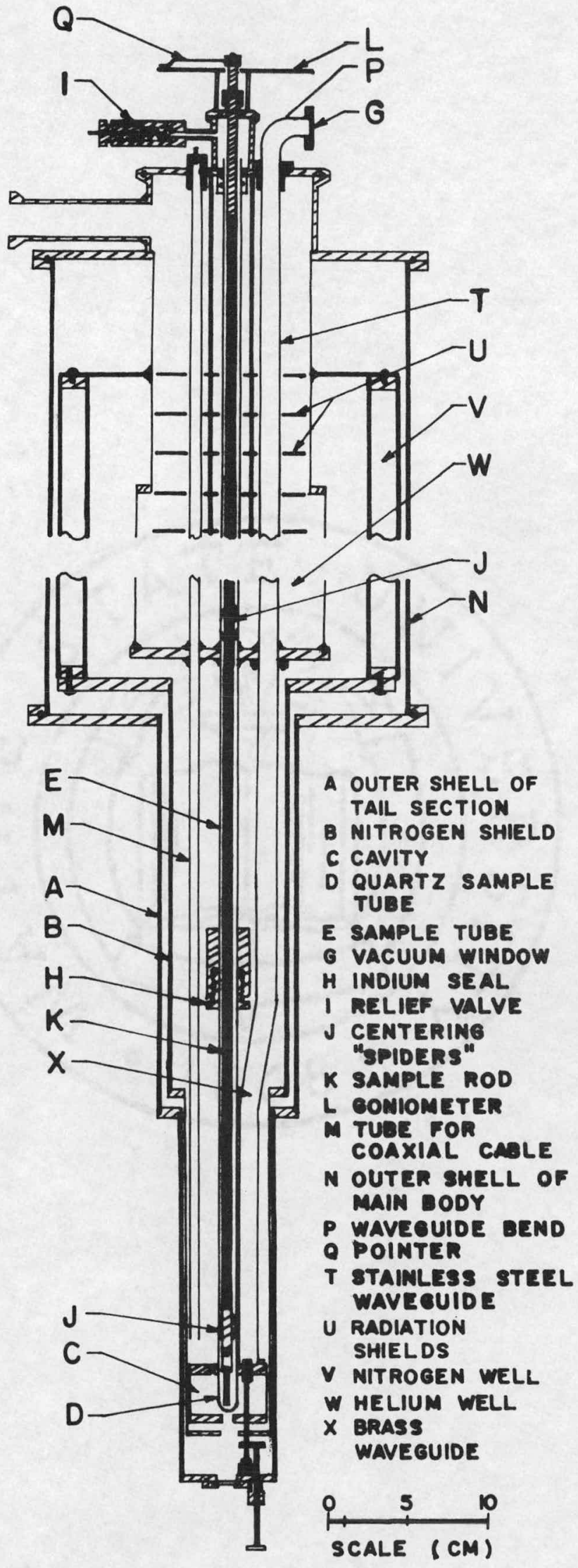

Fig. 10. Scaled schematic drawing of the complete helium cryostat. 
general varies between the derivative of a Gaussian line shape and the derivative of a Lorențian lịne shape. 11

\section{Helium Cryostat}

In order to study low temperature effects on the EPR signal, some sort of cryostat is necessary. This section describes in detail a cryostat that was designed, built, and successfully employed in the course of the present thesis work. The details of this cryostat were previously published by this author and Y.W. Kim in The Review of Scientific Instruments. 22

A schematic diagram of the cryostat used for all the $4.20 \mathrm{~K}$ measurements is given in Fig, 10. The outer shell of the main body $N$ and the liquid nitrogen reservoir are part of a standard modular cryostat built by Andonian Associates, Inc., Waltham, Massachusetts.

\section{Basic Mechanical Construction}

The outer shell of the cryostat tail A is made of brass, and is fastened to the main body by an 0-ring seal. The copper radiation shield $B$ is bolted to the bottom of the liquid nitrogen reservoir. The construction allows easy removal of the tail and access to the microwave cavity $C$ and the quartz sample tube $D_{0}$ The cavity and supporting waveguide are bolted to the bottom of the helium well. A $0.635 \mathrm{~cm}$ (1/4 inch) o.d. stainless steel coaxial cable tube, a $0.953 \mathrm{~cm}\left(3 / 8\right.$ inch) o.d. stainless steel sample tube $E_{0}$ and an $X$ band stainless steel wavegulde, all of which pass through the helium well, are soldered in the copper bottom of the hellum well and sealed by 0-rings at the top: This allows the top works to be easily removed. Figure 11 illustrates a section of the tail taken 
at right angles to Fig。10。 It shows an additional $0.953 \mathrm{~cm} 0$ 。d。 stainless steel tube $F$ so situated that during transfer of liquid helium the transfer tube extends down to within a centimeter of the cavity. This tube is attached to the relatively massive waveguide and cavity by copper braid at several locations. This technique permits rapid and efficient cooling of the large heat reservoir presented by the waveguide and cavity。

\section{Microwave Assemb1y}

An $\mathrm{X}$ band microwave bridge is attached at the top of the cryostat to a $90^{\circ} \mathrm{E}$ bend $\mathrm{P}$ as shown in Fig。10。 This waveguide bend has a vacuum tight window at one end $G_{0}$ and is sealed by an 0 -ring to the top works of the cryostat at the other end. The same 0-ring seals the stainless steel waveguide in the helium well to the top works o This construction allows the entire section of waveguide and the cavity to be part of the vacuum jacket of the cryostat. For rigid construction the waveguide connected to the bottom of the helium well is copper alloy and the cavity is brass. The cavity is cylindrical and operates in the $\mathrm{TE}_{011}$ mode. To prevent unwanted modes of oscillation in the cavity the top and bottom of the cavity are electrically insulated from the side wall by Teflon gaskets. The cavity is coupled to the waveguide by a mechanism similar to that of Ager, Cole, and Lambe ${ }^{23}$ The difference is that the electric field in the wavegulde is coupled to the magnetic field in the cavity。 Figure 12 shows the details of the coupling. A copper wire in a Teflon rod which can be rotated and moved up and down serves to vary the coupling. The Teflon rod extends out through the bottom 
of the cavity and is connected to a gear assembly, which subsequently comes out the bottom of the cryostat through an 0 -ring coupling. The gears unscrew from the tuning rod to allow removal of the liquid nitrogen shield. This arrangement permits the waveguide to be coupled to the top of the cavity and allows the diameter of the cryostat tail to be kept to a minimum。 (For rotating magnets the cryostat requires a minimum gap of $7.38 \mathrm{~cm}$ and for a fixed configuration of the cryostat and magnet a gap of $6.22 \mathrm{~cm}$ is required.)

\section{Optical Characteristics}

As seen in Fig。 11, the tail section has three sets of windows 2. two radially opposed sets of windows and a third set on the bottom of the tail. The outer windows are flat quartz disks sealed to the outer shell with rubber 0-rings. Since the vacuum jacket is common for the entire cryostat, $0.64 \times 1.9 \mathrm{~cm}$ rectangular holes in the liquid nitrogen shield $B$ suffice for windows. The radial windows $Y$ in the microwave cavity $\mathrm{C}$ are a series of closely spaced horizontal slots in the side wall of the cavity. Since the cavity resonates in the $\mathrm{TE}_{011}$ mode, this type of window offers a minimum perturbation to the microwave currents, which travel only in a horizontal direction in the outer wallo 24 The third window is simply a hole in the bottom of the cavity。

\section{Sample Positioning Mechanism}

As shown in Fig。 10 , the quartz sample tube $D_{\text {, }}$ which extends into the cavity, is attached by means of an indium $0-r i n g$ seal $H$ to the stainless steel sample tube which extends through the helium well 


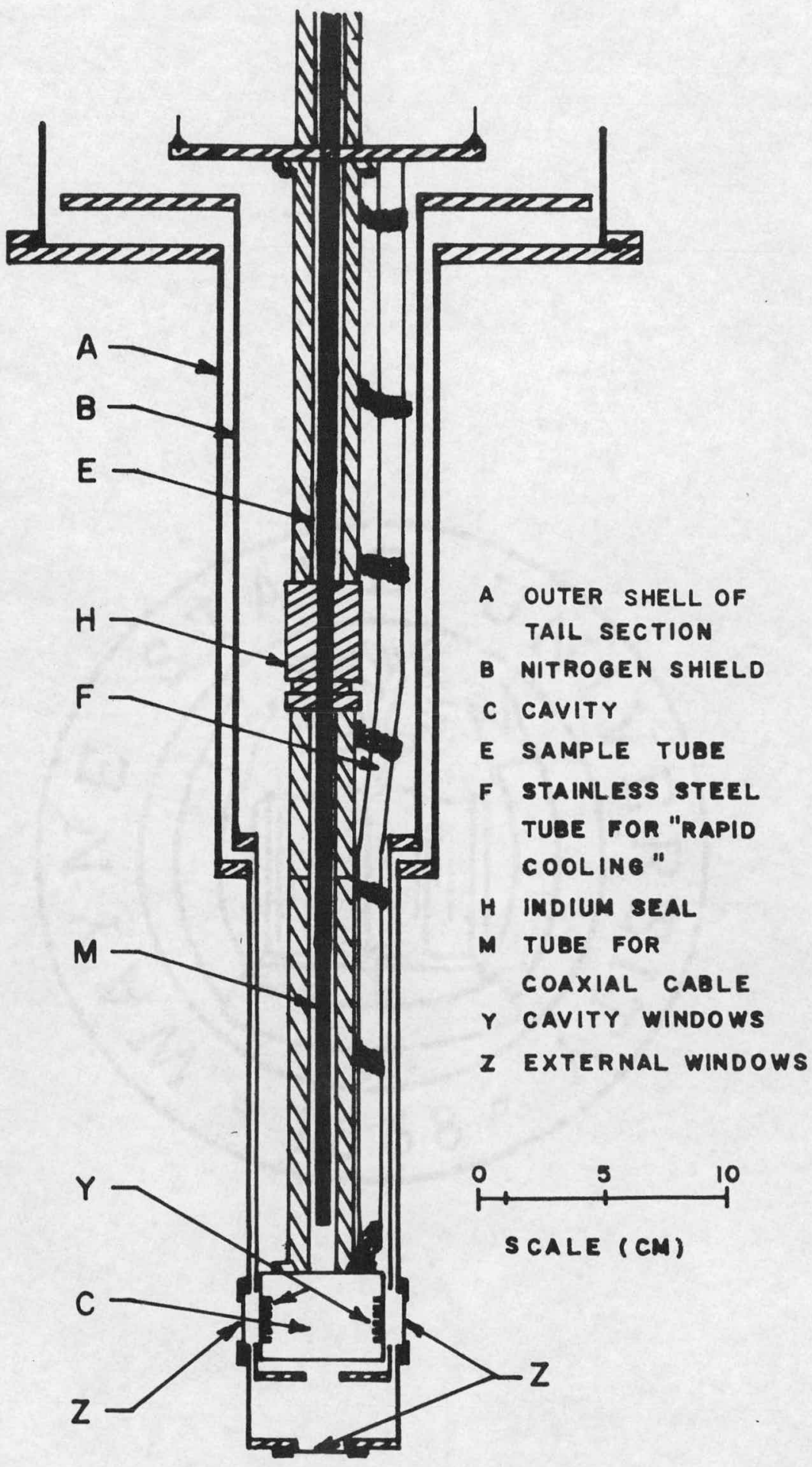

Fig. 11. Section of cryostat tail taken at right angles to the section given in Fig. 10 . 
to the top works of the cryostat. For operation with a cold finger the quartz tube would be replaced by a copper tube sealed at the bottom and extending down to the top of the microwave cavity. Only the sample; which 1s attached to the copper, extends into the cavity. At the top of the cryostat is an adjustable relief valve $I$ and a vacuum valve (not shown) for evacuating the sample tube. When using the quartz sample tube, the sample is attached to the end of a $0.635 \mathrm{~cm} \mathrm{o.d.} \mathrm{stainless}$ steel tube $K_{0}$ which extends from the cavity to the top of the cryostat。 A pointer $Q$ on top of the sample rod indicates the sample orientation on a goniometer $\mathrm{L}$, on top of the cryostat. The sample rod is kept centered in the sample tube with brass "spiders" J as shown in Fig。10。 The goniometer and the cap on the sample tube are removable to facilitate changing samples. This cap also has three electrical feedthroughs for thermocouple leads or carbon resistor thermometers.

The present experiments require a temperature of $4.2^{\circ} \mathrm{K}$ 。 Hence。 several small holes have been made in the sample tube in the helium well area to maintain liquid helium in the sample tube. For operation at temperatures above $4.2^{\circ} \mathrm{K}$ the sample tube would be a closed system。 The temperature of the sample would depend on the pressure of helium exchange gas in the sample tube.

ENDOR* ${ }^{*}$ Co11

A piece of $0.635 \mathrm{~cm} \mathrm{ood}$ o stainless steel tubing $M_{2}$ as shown in

* Electron-nuclear double resonance (ENDOR) is discussed in Ref。11。 This section is included in order to present a complete description of the cryostat. ENDOR experiments are not a part of the present thesis。 
Fig。 10, carries coaxial cable for the ENDOR coil in the cavity。 The coil itself is two or three turns of No. 24 copper wire attached to the quartz sample tube with thin strips of Teflon tape. One end of the coil is grounded in a threaded hole $S$ as shown in Fig. 12。 The other end passes through a hole $R$ in the top of the cavity.

\section{Discussion}

The modular design of the cryostat makes it extremely versatile and provides several interesting features. First, the tall section is readily removed. If the sample tube must be replaced or exchanged for the cold finger, a vacuum tight indium seal can be made with little or no trouble. Careful cleaning of the surfaces involved is the only precaution necessary。 Secondly, the waveguide and cavity are evacuated, thus preventing noise due to boiling liquid helium or foreign material that may be in the helium well. Also, since the cavity is maintained at near helium temperatures, it acts as an effective heat shield for the sample inside the cavity。 Thirdly, since a cylindrical cavity is used, the sample, which is on the axis of the cavity, can be rotated in the cavity。 The magnet can also be rotated around the cryostat。 Furthermore, the sample can easily be removed and another inserted. After changing samples the cavity coupling can be readjusted from outside the cryostat, Fourth, the windows allow for visual alignment of the sample in the cavity。 Fifth, when operating with a cold finger, one of the quartz windows could easily be replaced by a beryllium window to allow the sample to be $x$-irradiated while in the cavity and at low temperatures.

For most efficient use of liquid helium, it has been found that 


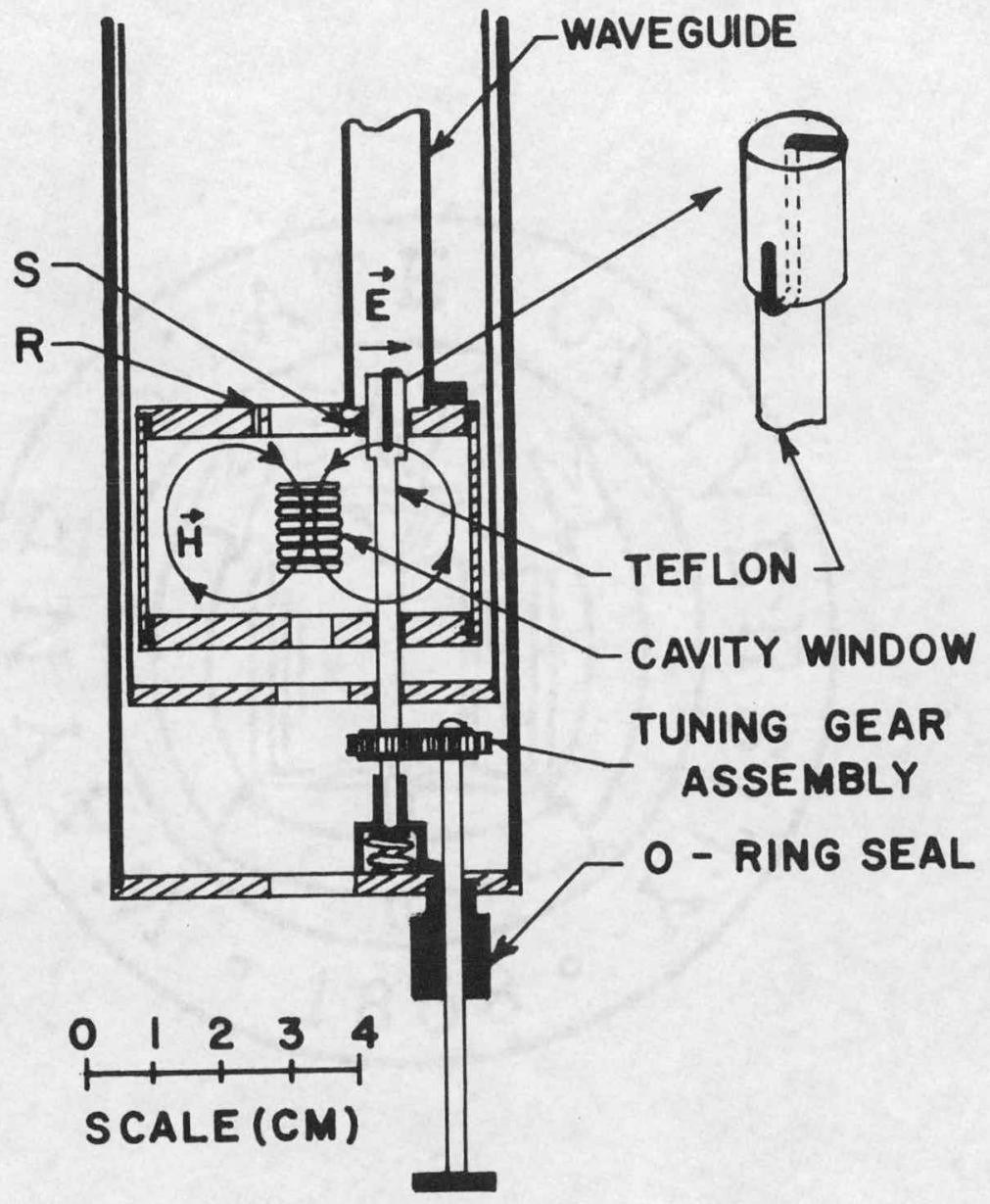

Fig. 12. Details of the microwave cavity and the coupling mechanism. 
transferring at a rate of about 6 liters/h into the bottom of the tube F until liquid starts to collect and then transferring at about 1 itter/min gives maximum efficiency. About 3 liters of liquid helium are required to cool the helium well (precooled with liquid nitrogen) to $4.2^{\circ} \mathrm{K}$ 。 Styrofoam in the neck of the cryostat prevents the venting helium from freezing out the 0-ring seals in the top works during a transfer. The helium well holds about 4 liters. With the three windows open and hellum in the sample tube the evaporation rate is about 0.2511 ter $/ \mathrm{h}$ 。 
CHAPTER IV

ELECTRON PARAMAGNETIC RESONANCE OF $\mathrm{Fe}^{3+}$ IN NaF*

\section{Introduction}

The work described in this chapter is concerned with EPR absorption studies of iron doped $\mathrm{NaF}$ at controlled temperatures below $180^{\circ} \mathrm{K}$. It is the purpose of this work to determine the characteristics of iron as an impurity in $\mathrm{NaF}$. The results of this type of study are necessary before an investigation of the effects of iron on the optical bleaching process can be made.

The paramagnetic resonance of Iron impurity in NaF has been investigated by Bleaney and Hayes, 25 and Hayes and Jones. 26 These authors have observed an EPR absorption due to $\mathrm{Fe}^{+}$only. The EPR absorption of $\mathrm{Fe}^{3+}\left({ }^{6} \mathrm{~S}_{5 / 2}\right)$ has been observed in several iron-doped single crystals c7-28 $^{2}$ other than alkali halides. However, no successful observation of the EPR absorption of $\mathrm{Fe}^{3+}$ seems to have been made in alkali halldes.

In the present Chapter, experimental evidences will be presented, which indicate strongly that the ionic state of the iron impurity of $\mathrm{NaF}$ may be changed from $\mathrm{Fe}^{2+}$ into a singly-ionized state $\left(\mathrm{Fe}^{+}\right)$and a triple-ionized state $\left(\mathrm{Fe}^{3+}\right)$ by means of $\mathrm{x}$-irradiation of the iron-doped $\mathrm{NaF}$. Both $\mathrm{Fe}^{+}$and $\mathrm{Fe}^{3+}$ appear to be located substitutionally at cation sites and to interact with the crystalline field as well as the six nearest nelghbor fluorine ions. In particular, the interaction of the $\mathrm{Fe}^{3+}$ ion with its environment is remarkably

The content of this Chapter is based on a manuscript submitted to the Physical Review by this author and Y.W. Kim. 
temperature-dependent. The spin Hamiltonian for the $\mathrm{Fe}^{3+}$ has been determined in such a way that it can account for the observed temperature dependence. The results are compared with the current theory of the fine structure splitting of S-state ions.

\section{Experimentals}

Single crystals of NaF were obtained from the Harshaw Chemical Co。 Cleveland, Ohio. Mass spectroscopic analysis* of these crystals indicated that the crystal samples contained 8 parts per million (ppm) of iron, 3 ppm of manganese, but no detectable amount of chromium $(<1 / 10 \mathrm{ppm})$ 。 Samples of dimensions approximately $3 \mathrm{~mm} \times 3 \mathrm{~mm} \times 15 \mathrm{~mm}$ were cut from these crystals in such a way that the longest dimension of the samples is parallel to the [110]-direction.

The samples were then irradiated by $x$-rays from a molybdenum target tube operated at $50 \mathrm{Kv}$ and $20 \mathrm{ma}$ for about 90 hours at room temperature。

The (EPR) spectra of these samples before and after the irradiation were investigated near $77^{\circ} \mathrm{K}$ and $4.2^{\circ} \mathrm{K}$ 。 The EPR spectrometer used for these studies is a Varian model V4500 X band spectrometer with 100 kcps and audio frequency magnetic field modulation (See Chapter III)。The 11quid helium cryostat described in Chapter III has been employed for parts of the present work。

The angular dependence of the spectra is investigated by rotating the samples about the [110]-axis which is held perpendicular to the $D_{0} C$ 。 and microwave magnetic fields. The angle $(\theta)$ of rotation is measured from the [110]-direction of the samples to the direction

\footnotetext{
*- Spectroscopic analysis was performed by the Harshaw Chemical Co.
} 
of the D.C. magnetic field, which remains on the (110)-plane。

Experimental Results and Spin Hamiltonian

The samples have yielded weak EPR spectra of the 3 ppm of manganese 1mpurity before the x-irradiation, but no trace of the EPR absorption of the $8 \mathrm{ppm}$ of iron impurity. The lack of the resonance of the iron impurity is not unexpected, since the iron enters the lattice of $\mathrm{NaF}$ as a divalent $1 \mathrm{In}^{29}\left(\mathrm{Fe}^{2+}\right)$ and the EPR resonance of $\mathrm{Fe}^{2+}$ is very sensitive to the lattice defects, 30 making the width of the resonance so broad as to be practically unnoticeable.

The $x$-irradiation has been found to reduce the intensity of the manganese resonance to practically $n 11$, and to Induce three EPR spectra which are easily distinguishable at different temperatures.

Near $77^{\circ} \mathrm{K}$, only two of them are observable. One represents the well-known F center resonance. 31 The intensity of this $F$ center resonance, however, can be reduced substantially by optically bleachIng the $x$-irradiated samples with F-light (340 mu). After the optical bleaching, then, the other of the two spectra is better identified. This second spectrum is centered approximately at $g=2.002$, and exhibits a partially resolved anisotropic structure. The solid curves of Fig. 13 illustrate four spectra recorded at $\theta=0^{\circ}, 34^{\circ}, 55^{\circ}$, and 900。 $\theta=0^{\circ}, 55^{\circ}$, and $90^{\circ}$ correspond to [100], [111], and [110] respectively. The best resolution is obtained for $\theta=34^{\circ}$ and $90^{\circ}$, while poorer resolutions are noticed for $\theta=0^{\circ}$ and $55^{\circ}$. For the orientations of best resolution, the spectrum consists of seven lines, which are approximately equally spaced (13.7 gauss apart). The intensity ratio of the seven lines is approximately $1: 6: 15: 20: 15: 6: 1$ 。 

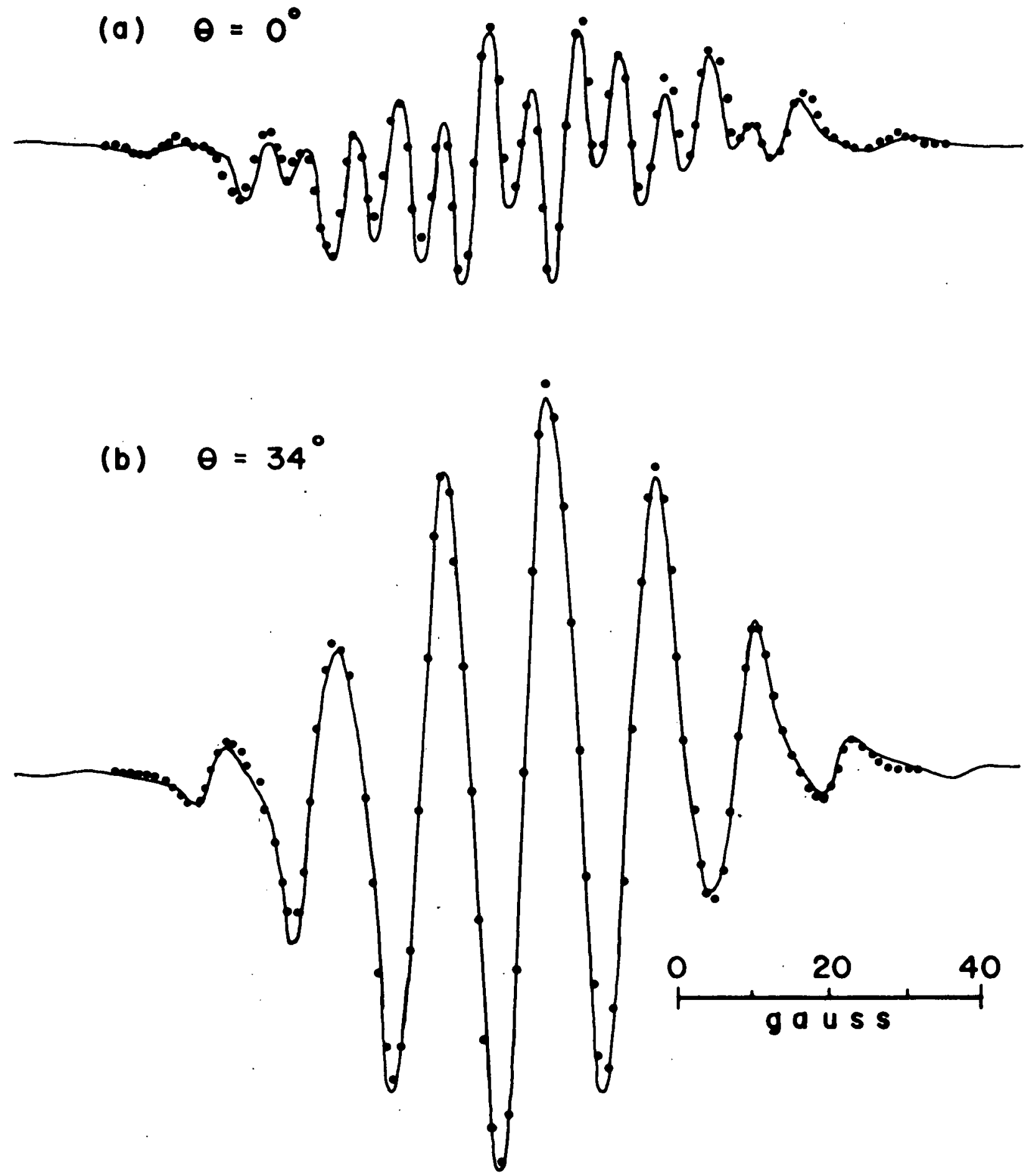

Fig. 13. The observed EPR spectra (solfd lines) and the theoretical fit (dots) of $\mathrm{Fe}^{3 \mathrm{~F}}$ in $\mathrm{NaF}$ at $77^{\circ}$ for $\theta=0^{\circ}, 34^{\circ}, 55^{\circ}$, and $90^{\circ}$. (Continued on next page.) 

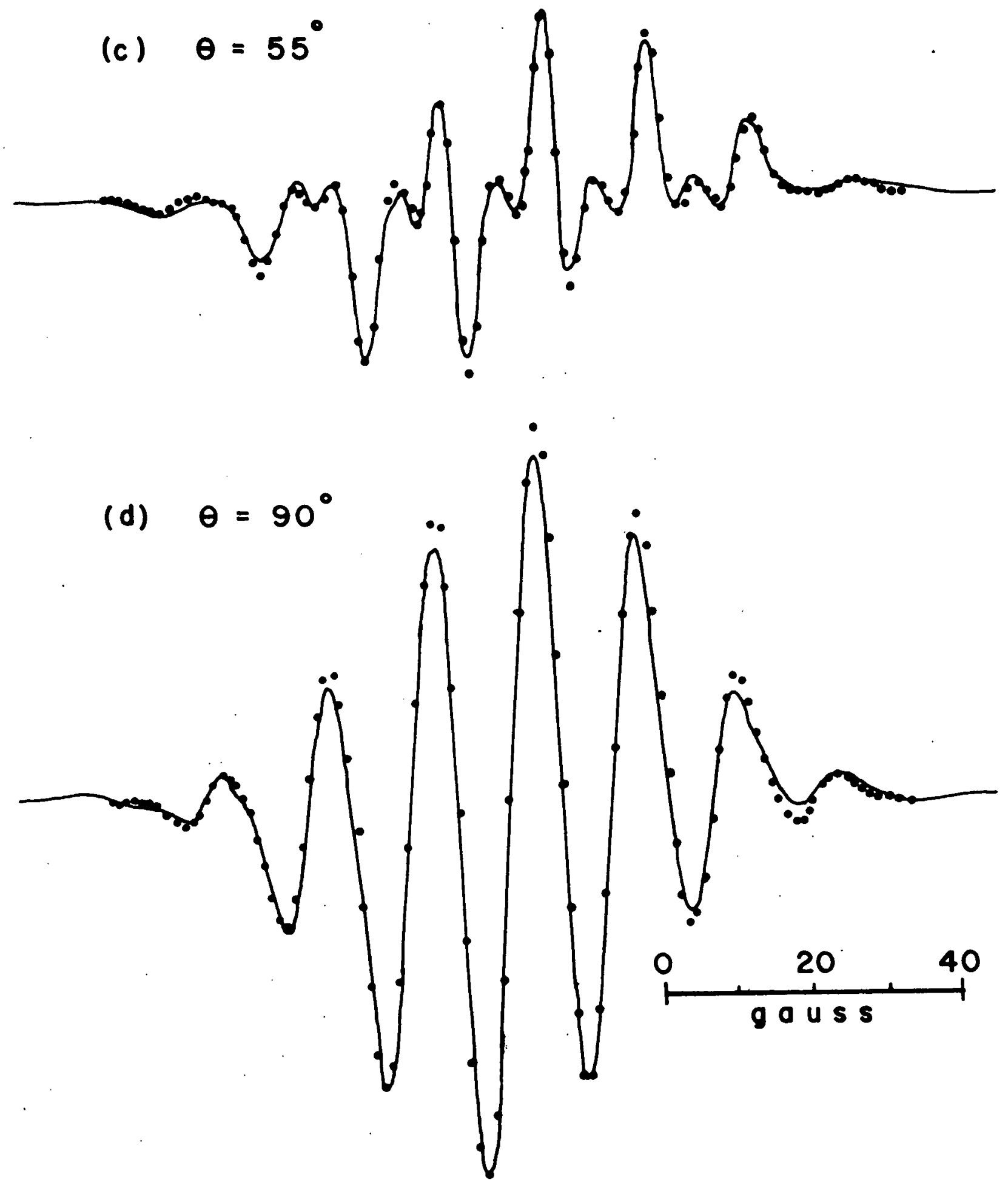

Fig. 13. (Continued). 
Near $4.2 \% \mathrm{~K}$, the second spectrum is not observable, and the $\mathrm{F}$ center resonance has been already reduced by the optical bleach. Instead, a third spectrum with a partially resolved anisotropic structure is noticed. This one is centered near $g=4.344$ 。 The anisotropy of this spectrum is different from that of the second spectrum, and has been found to be satisfactorily described in terms of the spin Hamiltonian that Bleaney and Hayes ${ }^{25}$ previously assigned to $\mathrm{Fe}^{+}$located at a cation site in $\mathrm{NaF}$. In the remaining part of this paper, therefore, only the second anisotropic spectrum will be dealt with。

As preliminary clues to the determination of the proper spin Hamiltonian for describing the spectrum, two points of observation have been used: First, the intensity ratio of the seven lines mentioned in the preceding is very close to that which would be expected from six equivalent nuclei of spin $I=1 / 2$, each interacting with the paramagnetic ion responsible for the spectrum。 In the present case。 $\mathrm{Na}^{23}$ and $\mathrm{F}^{19}$ are both $100 \%$ naturally occurring isotopes with nuclear spins of $3 / 2$ and $1 / 2$ respectively。 This suggests that the paramagnetic ion be situated on a cation site with cubic symmetry and interact with six equivalent nearest=neighbor fluorine 1ons. Secondly, if the resolved spectrum is due solely to the hyperfine interaction (see Appendix I)。 the resolution should be best for $\theta=55^{\circ}$, when the anisotropic part of the hyperfine interaction vanishes and leaves only

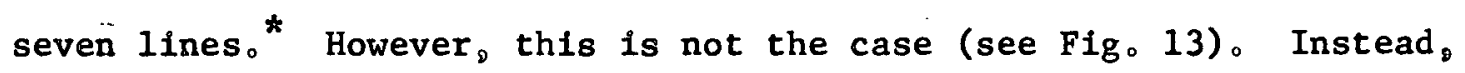

* This is the case for cubic symmetry when $\vec{H} / /[111]$; then $\left(3 \cos ^{2} \theta_{n}-1\right)=0$ for $n=1$ 。०。 6 。 
the observed angular dependence of the resolution appears to be more similar to what would be expected from unresolved fine structure splitting (see Appendix II) of an ion in the ${ }^{6} \mathrm{~S}_{5 / 2}$ state ${ }^{32}$ Thus, the spin Hamiltonian for the spectrum is taken to be:

$$
\mathcal{H}_{s}=g \beta \vec{H} \circ \vec{S}+\sum_{n=1}^{6} \vec{S} \circ \frac{A}{n} \circ \vec{I}_{n}+F(a)
$$

The first term of $\mathrm{Eq}$. (10) describes the Zeeman interaction of the electron spin $(S=5 / 2)$ with the magnetic field $H_{\diamond}$ where the spectrom scopic splitting factor $g=2.002$, and $\beta$ is the electronic Bohr magneton。 The second term of Eq。(10) denotes the summation of the hyperfine interaction tensor $\frac{A}{n}$ of the $n-t h$ fluorine nucleus of spin $I_{n}(=1 / 2)$ over the six nearest neighbor fluorine nuclei。

Since the paramagnetic ion is on a cubic lattice site, each of the hyperfine tensors is axially symmetric and the hyperfine term can be written as follows (see Appendix I):

$$
\sum_{\mathbf{n}} \cdot I_{n z^{\circ}} S_{z^{0}}\left[\delta+\gamma\left(3 \cos ^{2} \theta_{n}-1\right)\right]
$$

where the subscript $z^{0}$ refers to the direction of the external field along which $\overrightarrow{\mathrm{S}}$ and $\overrightarrow{\mathrm{I}}_{\mathrm{n}}$ are quantized ${ }^{3}$ and $\theta_{n}$ is the angle between the direction of the magnetic field and the axis joining the $n-t h$ fluorine nucleus to the paramagnetic ion. The two Greek letters $\delta$ and $\gamma$ represent the isotropic and anisotropic (hyperfine) coupling constants respectively。

The third term of $\mathrm{Eq} \circ(10)$ is the fine structure term for the ${ }^{6} \mathrm{~S}_{5 / 2}$ ion in a cubic crystalline field。 It has the following form (see Appendix II): 


$$
F(a)=(1 / 6) a\left[s_{x}^{4}+s_{y}^{4}+s_{z}^{4}-(1 / 5) s(s+1)\left(3 s^{2}+3 s-1\right)\right]
$$

where the suffices $x_{0} y_{0}$ and $z$ refer to the crystalline axes, and $a$ is the fine structure constant. The fine structure term depends only on the electron spin operators, and splits transitions between the electron spin levels as follows (see Appendix II):

$$
\begin{array}{ll}
M_{S}= \pm 1 / 2 \rightarrow \mp 1 / 2: & H=H_{0} \\
M_{S}= \pm 3 / 2 \rightarrow \pm 1 / 2: & H=H_{0} \pm(5 / 2) p(a / g \beta) \\
M_{S}= \pm 5 / 2 \rightarrow \pm 3 / 2: & H=H_{0} \pm 2 p(a / g \beta)
\end{array}
$$

where $H_{0}=\frac{h \nu}{g \beta}, \nu$ being the fixed microwave resonance frequency, and $p=(1 / 5)\left(l^{2} m^{2}+m^{2} n^{2}+n^{2} \ell^{2}\right)$ with $\ell_{\diamond} m_{\diamond} n$ the direction cosines of $\vec{H}$ referred to the cubic axis of the crystal. The transitions given by Eqs。(13)。(14)。 and (15) have an intensity ratio of 9:8:5 respectively。 Using the spin Hamiltonian given in $\mathrm{Eq} 。(10)$, theoretical spectra were calculated with the aid of an IBM 7074 computer ( $W_{0} S_{\circ} U_{0}$ Computing \& Data Processing Center, Detroit, Michigan)。The first step in this calculation is to determine the magnetic energy-level structure of $f_{s}$. The Zeeman term ylelds six equally spacedelectron spin levels and each of them is split into the nuclear hyperfine structure determined by the hyperfine term。 In general, there are:64 hyperfine levels。 each corresponding to one of the $\left(2 I_{n}+1\right)^{6}$ possible orientations of the six nuclear spins, each $I_{n}=1 / 2$. Finally, the fine structure term splits each of these into five levels, From this structure, then, the energy separation $\Delta E_{i \delta}$ of all the allowed transitions is determined along with the magnetic field value $H_{i}=\Delta E_{i} / g \beta$, at which the transition will take place. Knowing all possible values of $H_{i}$ it is then possible to 
construct a theoretical spectrum。 Using the magnetic field, $\mathrm{H}$, as the abscissa, the derivative of a Gaussian 1 ine, ${ }^{*}$ which has a width, $W$, between the points of maximum slope, is placed at all values of $H_{i}$ Each of these Gaussian lines is weighted by the appropriate intensity factor。 The individual Gaussian derivative curves are then superimposed and added togther at .5 gauss intervals to obtain the overall composite spectrum. The interval of summation (.5 gauss) is chosen to obtain the maximum resolution in the computed spectrum. The structure and anisotropy of the computed spectrum naturally depends on the values of the parameters $\left(\delta, \gamma, a_{,}\right.$and $\left.W\right)$. These parameters are varied over wide ranges, so that the resulting computed spectra fit well the observed spectra。 Two facts are especially helpful in determining the best fit: (1) for $\theta=55^{\circ}$ the anisotropic hyperfine interaction vanishes and has no effect on the spectrum, and (2) for $\theta \simeq 30^{\circ}$ the fine structure splitting vanishes ${ }^{+}(p=0)$ and a has no effect on the spectrum.

The results of this computer fit for the observed spectra at $77^{\circ} \mathrm{K}$ are 1llustrated in Fig。13, where the spectra for four values of $\theta\left(0^{\circ}, 34^{\circ}, 55^{\circ}\right.$, and $\left.90^{\circ}\right)$ are compared. The solid lines are the observed spectra and the dots represent the computed spectra. The values of the spin. Hamiltonian parameters which: gave.this fit are given

* Gaussian shaped lines were used in this calculation since there is probably some broadening due to the hyperfine interaction with next nearest neighbor nuclei: Also, the individual lines making up the composite spectra are probably broadened by thermal vibrations as will be shown later on in this chapter.

+ Experimentally the spectra reaches a maximum amplitude for $\theta$ near $34^{\circ}$ even though the fine structure disappears near $30^{\circ}$. This is probably due to the anisotropic hyperfine interaction which is decreasing at this point and vanishes when $\theta=55^{\circ}$ 。 
in Table 1. The agreement between the observed spectra and the calculated spectra is remarkably satisfactory and justifles the reasoning that led to the choice of the electronic state of the paramagnetic ion $\left({ }^{6} S_{5 / 2}\right)$ in a cubic site and its spin Hamiltonian.

\section{Temperature Effects}

As the temperature of the samples is raised from $77^{\circ} \mathrm{K}$ to $180^{\circ} \mathrm{K}_{8}$ * the features of the spectra for different angles change quite rapidly and significantly。 For example, near $180^{\circ} \mathrm{K}$, the spectra for all values of $\theta$ tend to show only a seven-line pattern as is observed for $\theta=90^{\circ}$ at $77^{\circ} \mathrm{K}$, and there is an accompanying decrease of the spectral amplitude. The solid curves of Fig. 14 illustrate the spectra for $\theta=55^{\circ} \mathrm{K}$ and $\theta=90^{\circ}$ near $180^{\circ} \mathrm{K}_{8}$ and the solid curves of Fig. 15 show the observed temperature dependence of the amplitude of the two spectra in Fig. 14 .

A comparison of the spectrum for $\theta=55^{\circ}$ of $\mathrm{Fig} .14$, for example。 with the corresponding spectrum in Fig。 13 would indicate easily the interesting effect of the temperature change. The structural change of the spectra at $180^{\circ} \mathrm{K}$. Indicates that the spin Hamiltonian parameters are changing and that the temperature effect is not solely due to the Boltzmann temperature dependence of the spin level population. Another evidence in support of this view is given by the observed temperature dependence of the spectral amplitude. The portion of the amplitude change due to the Boltzmann temperature dependence (of the spin level populations) can be readily removed by means of a correction factor.

* At higher temperatures, the spectral intensity at $\theta=0^{\circ}$ becomes quite small and weak signals due to unidentifiable impurities make a detailed analysis of this spectrum difficult. 
TABLE 1

SPIN HAMILTONIAN PARAMETERS FOR THE OBSERVED RESONANCE DUE TO $\mathrm{Fe}^{3+}$ IN NaF AT $77^{\circ} \mathrm{K}$

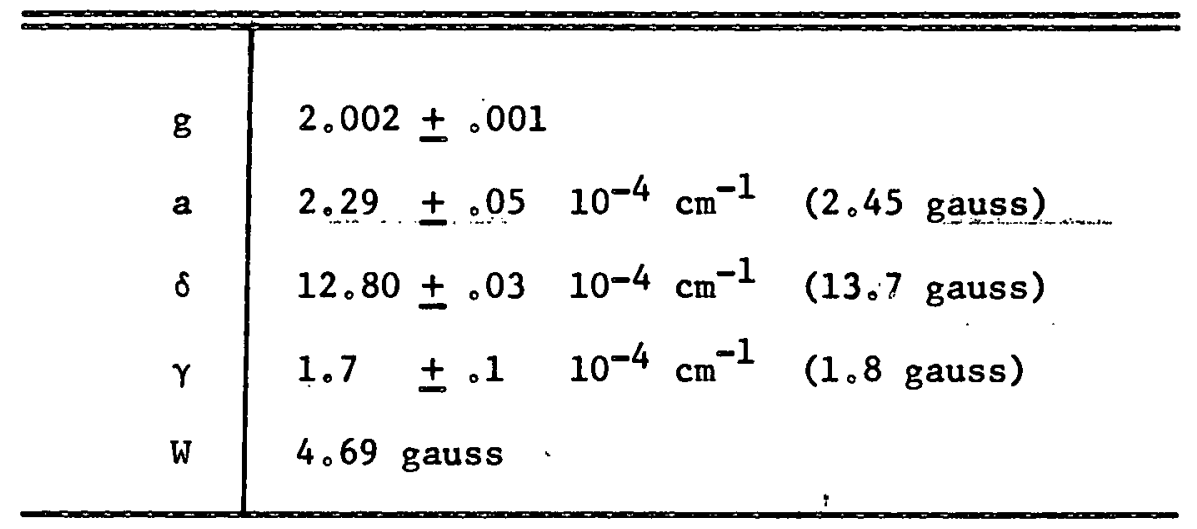



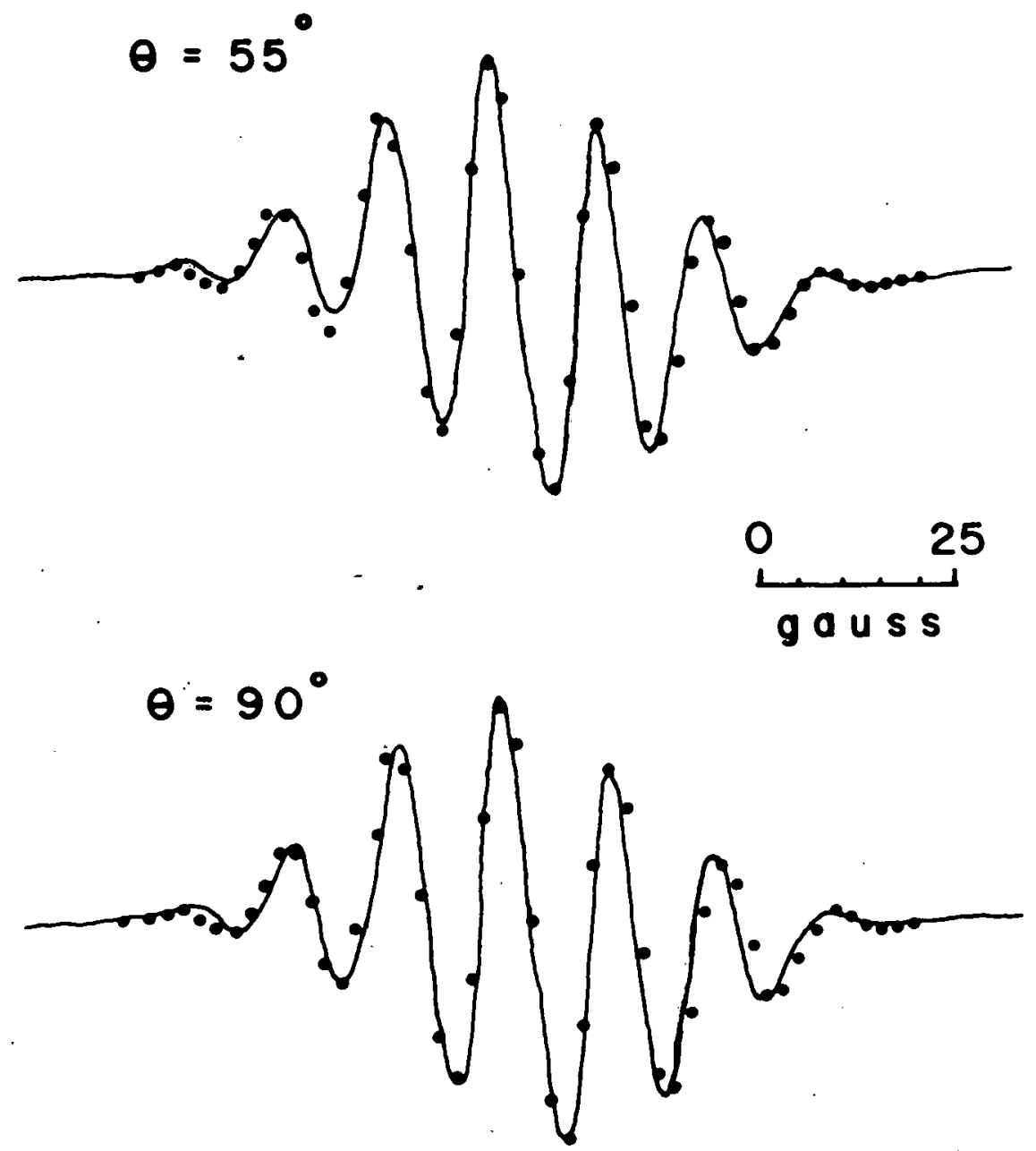

Fig. 14. The observed EPR spectra (solid lines) and the theoretical fit (dots) of $\mathrm{Fe}^{3+}$ in NaF near $180^{\circ}$ for $\theta=90^{\circ}$ and $\theta=55^{\circ}$. 


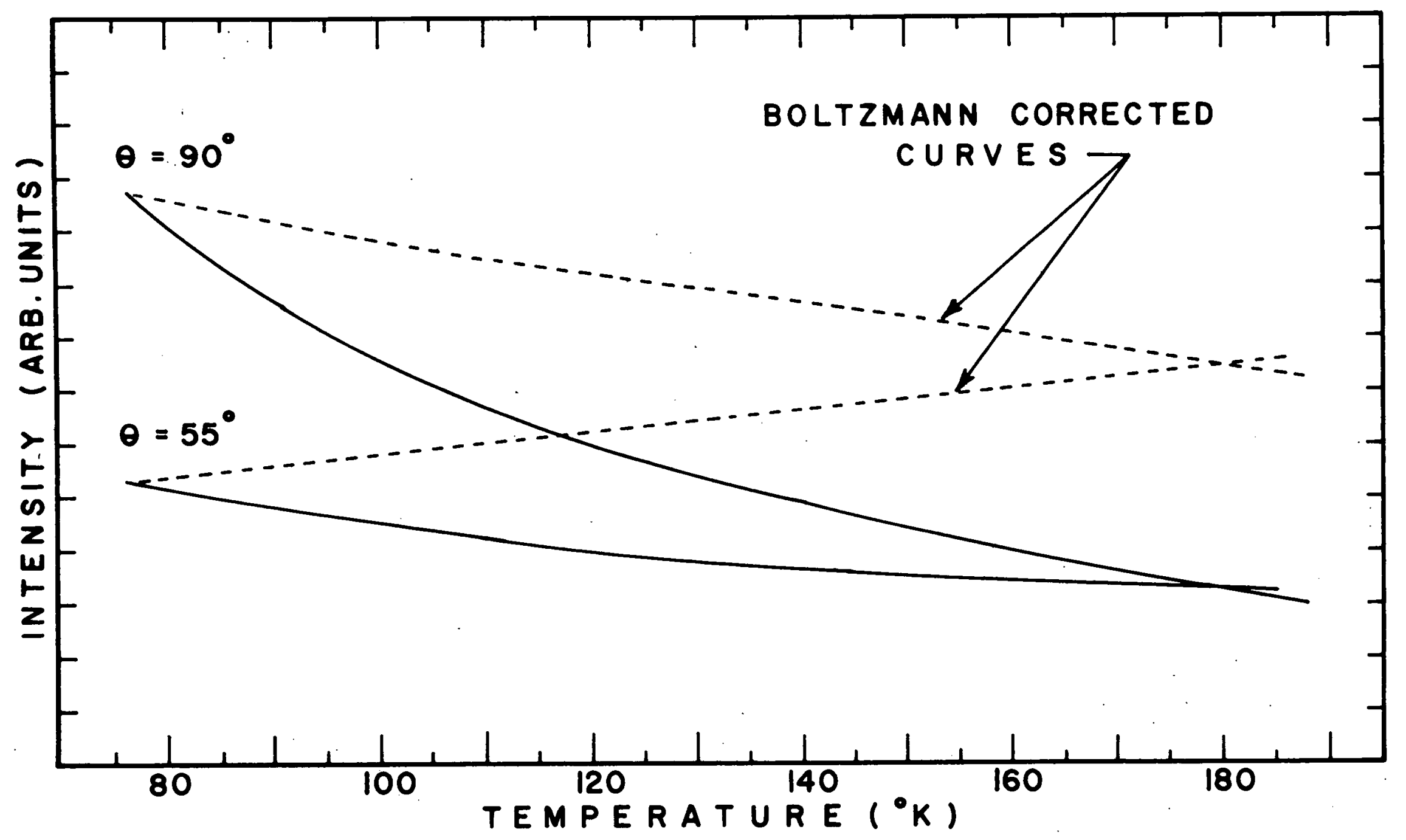

Fig. 15. The observed temperature dependence of the EPR spectral amplitude for $\theta=90^{\circ}$ and $\theta=55^{\circ}$ (solid curves). The dashed curves represent the experimental curves corrected for the Boltzmann temperature dependence. 
When the correction is made, the solid curves in Fig。 15 should behave like the two dashed curves. If the amplitude change were due solely to the Boltzmann temperature dependence, the dashed curves would have to be horizontal. The fact that they are not indicates that the spin Hamiltonian parameters and/or $W$ are changing, as the temperature is varied。

A major consideration in the computer analysis of the temperature dependence of these spectra is, therefore, that the computed spectra show only the basic seven-line pattern near $180^{\circ} \mathrm{K}$ and that the anisotropy of the computed spectra corresponds to the observed anisotropy。 Otherwise, theoretical spectra for the higher temperatures were calculated in the same way as described previously for the $77^{\circ} \mathrm{K}$ spectra by adjusting the values of $a_{2} \gamma_{2}$ and $W_{0}$ All the computed spectra were normalized so that the area under the absorption curve remained constant for changes in $\mathrm{W}$ 。

The computation results have indicated that the observed change in the structure and amplitude of the spectra for different temperatures is primarily due to the temperature dependence of $a$ and $W_{2}$ and slightly due to $\gamma_{0}$ while $g$ and $\delta$ appear to remain constant within experimental errors. The results of the computation are illustrated by the dots in Fig。 14 . The agreement is satisfactory。 Figure 16 illustrates the computed temperature dependence of $a_{2} W_{2}$ and $\gamma_{0}$ which has been found to be satisfactory to account for the observed temperature dependence of the spectra. Three points of significance should be noticed: First, a decreases practically linearly as $T$ increases Second, $W$ is found to be proportional to the square root of $T_{0}$ Third。 


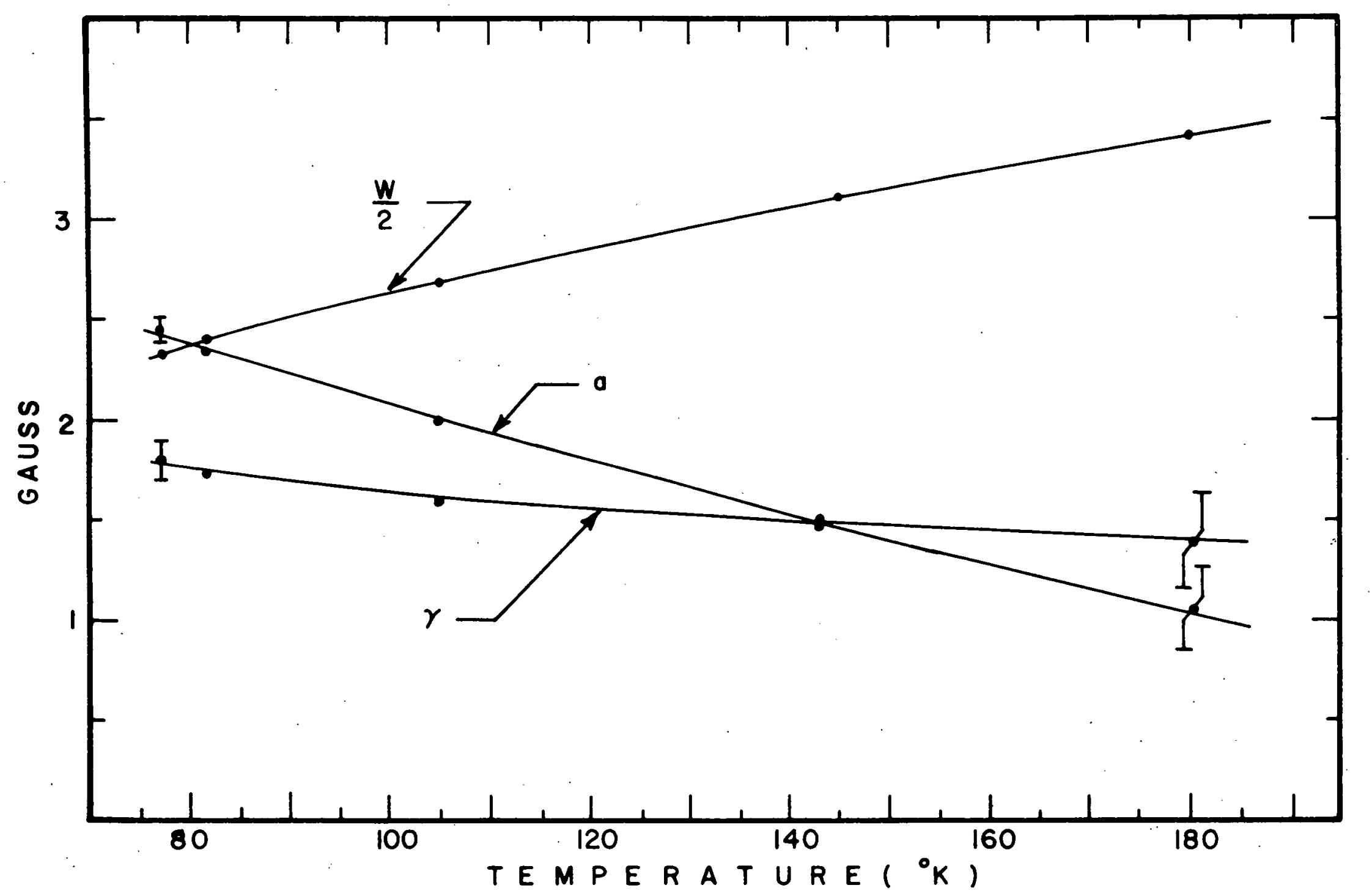

Fig. 16. The temperature dependence of $a, \gamma$, and $W$ found by fitting the experimental data at several temperatures. $g$ and $\delta$ are found to be constant within experimental error. 
$\gamma$ tends to decrease as $T$ increases, but the large error in the computam tional difficulty makes it doubtful whether the change of $\gamma$ is significant, as compared with the change of a and $W_{0}$

\section{Discussion}

The results of the analyses in the preceding show that the choice of the electronic state $\left({ }^{6} \mathrm{~S}_{5 / 2}\right)$ of the paramagnetic ion its location at a cation site surrounded by a cubic field, and the accompanying spin Hamiltonian Eq. (1) is very satisfactory in accounting for the anisotropy of the spectra as well as for the temperature dependence of the spectra。

Each of the three metallic impurities (1ron, manganese, and chromium) of the $\mathrm{NaF}$ samples investigated in the present work can exist in the ${ }^{6} \mathrm{~S}_{5 / 2}$ electronic state: $\mathrm{Fe}^{3+}{ }_{0} \mathrm{Mn}^{2+}$, and $\mathrm{Cr}^{+}$。 of the three possibilities, $\mathrm{Fe}^{3+}$ is most favorable. The following reasons may be cited。First, $\mathrm{Mn}^{2+}$ has a nuclear spin of $5 / 2$ and a large nuclear magnetic moment, which would lead to a large hyperfine splitting of the spectra and would contribute to the spin Hamiltonian in Eq。(1) an additional term representing the hyperfine interaction of the electronic spin with the nuclear spin of $\mathrm{Mn}^{2+}$. This additional term would then lead to a hyperfine splitting ( 290 gauss) of the spectra, which is not the case in the present work. Furthermore, the x-irradiation has reduced the resonance of $\mathrm{Mn}^{2+}$ practically to $\mathrm{nil}$ (see Section on Experimental Results and Spin Hamiltonian)。 Secondly, the concentration of the chromium impurity is too small (less than one-tenth ppm)。 to be responsible for the spectra, since the observed spectra corresponds to a concentration of at least $1 \mathrm{ppm}$ of impurity. Thirdly, the parametersfor $\mathrm{Cr}^{+}$in $\mathrm{NaF}$ reported by Hayes and Jones ${ }^{26}$ are not the 
same as the parameters determined in the present work。

The ionic radius of an $\mathrm{Fe}^{3+}$ ion is relatively small $(0.6 \AA)^{33}$ and the interatomic spacing of the NaF lattice is $2.31 \AA$. This might lead one to speculate that the $\mathrm{Fe}^{3+}$ should be located in an interstitial position as it is in $\mathrm{AgCl}^{27}$ The conclusion in the present work that it is not in an interstitial position is probably attributable to the stability ${ }^{34}$ of the complex $\left(\mathrm{FeF}_{6}\right)^{3-}$. This complex puts the iron in a lattice site with cubic symetry.

To preserve local electrical neutrality in the lattice, each $\mathrm{Fe}^{3+}$ should produce two cation vacancies. These vacancies will cause departures from cubic symmetry at the $\mathrm{Fe}^{3+}$ site (see $\mathrm{Fig}, 17(\mathrm{a})$ )。 if they are close to the $\mathrm{Fe}^{3+}$ site forming a complex. This would introduce additional nonocubic terms into the spin Hamiltonian, Eq。(10)。 Since the resonance can be fit by only cubic terms, the charge compensating vacancies are not ${ }^{00}$ bound" to the $\mathrm{Fe}^{3+}$ site (see Fig。17(b))。

The computed temperature dependence of a and W (see Fig. 16) facilitates some discussions with regard to the mechanism of the line broadening, and the existing theoretical expression for $a_{0}$

First, as mentioned in the preceding, the line width $W$ is fairly well proportional to $(T)^{1 / 2}$, where $T$ is the temperature. This depen dence may be explained in terms of the thermal vibration of the ions neighboring the $\mathrm{Fe}^{3+}$ ions. The thermal vibration is expected to cause fluctuations in the zeromield splitting, 35 which is measured by $a_{0}$

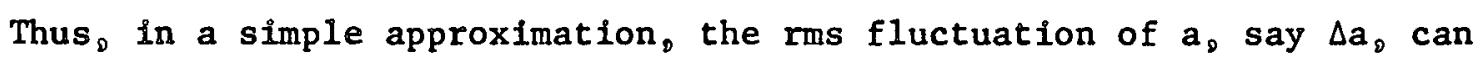
be taken to be a measure of $W_{0}$ and it can be taken to be proportional to the rms amplitude of the thermal vibration, say $\Delta x_{\text {. Therefore, } W}$ becomes proportional to $\Delta x$ 。 Now, the average energy of an ion in 
(a)

$$
\begin{aligned}
& +-+-+-+- \\
& -+-+-+-+ \\
& +-\mathrm{Fe}^{3+}+-+- \\
& -+-+-+-+ \\
& +-+-+-+- \\
& -+-+-t_{-}+ \\
& +-+-\mathrm{Fe}^{3+}+- \\
& -+-+-+-+
\end{aligned}
$$

(b)

$$
\begin{aligned}
& +-+-+-+- \\
& -+-+-\mathrm{Fe}^{3+}+ \\
& +-+-+-+- \\
& -+-+-+-+ \\
& +-+-+-+- \\
& -+-\mathrm{Fe}^{3+}+-+ \\
& +-+-+-+- \\
& -+-+-+-+
\end{aligned}
$$

Fig. 17. Schematic diagram showing (a) associated charge compensating cation vacancies in next-nearest neighbor positions forming a "complex," and (b) charge compensating vacancies away from the $\mathrm{Fe}^{3+}$ ion. In this case the $\mathrm{Fe}^{3 t}$ ions are left in a site with cubic symmetry and the vacancies are free to migrate at sufficiently high temperatures depending on the activation energy. 
thermal vibration is proportional to $(\Delta x)^{2}$ and also to $T_{0}$ Therefore。 $W$ becomes proportional to the square root of $T_{0}$

Secondly, the fine structure constant, $a_{0}$ decreases as $T$ increases。 This tendency is consistent with the existing speculation。 36 The decrease of a is practically linear in $T$. This linear relation leads to a linear dependence of a on the lattice constant $d_{2}$ for the case of the linear expansion of the lattice. Such a relationship between a and $d$ is expected of the lattice. Such a relationship between a and $d$ is expected from the discussions in the preceding。 Quantitatively, however, the amount of change (approximately $60 \%$ ) of a between $77^{\circ} \mathrm{K}$ and $180^{\circ} \mathrm{K}$ does not appear to be easily compatible with the existing theories on a. According to the theories on the $S \backsim s t a t e$ ions, the fine structure constant is given in a certain approximation by the following: 37

$$
a=\frac{K \lambda^{4}}{E_{P S}^{4}}
$$

where $K$-is the matrix element of the potential $V$ of the cubic crystalline field $d_{\theta}$ and $E_{P S}$ and $\lambda$ are the energy spacing between the ${ }^{4} P$ and ${ }^{6} S$ states of the free $\mathrm{Fe}^{3+}$ ion and the spin-orbit coupling constant respectively。 Since $E_{P S}$ refers to the free ion $_{0}$ the temperature dependence of a must come from $\mathrm{K}$ and/or $\lambda_{0}$ according to Eq。(16)。 of the two, $\mathrm{K}$ and $\lambda_{0}$ the latter seems to be less significant in the T-dependence of $a_{0}$ The reason is the following: Experimentally, the spectroscopic splitting factor, $g_{0}$ is practically insensitive to temperature changes in the range between $77^{\circ} \mathrm{K}$ and $180^{\circ} \mathrm{K}$ 。 This means that the change of $\lambda$ over the same temperas ture range, if any, is not significant. Thus, $\mathrm{K}$ becomes the major factor to produce the temperature dependence of $a_{0}$ The wave function used for the matrix element is more or less insensitive to the temperature change。 
Consequently possible $\mathrm{T}$-dependence of $\mathrm{K}$ originates from the temperature dependence of the cubic field potential vo simple calculations by means of $\mathrm{Eq} .(16)$ and a linear thermal expansion of the lattice produce about 2 to $3 \%$ change of a over this temperature range. This is just too small to account for the observed change $(60 \%)$ of $a_{0}$ It appears that in order to account for the large change of $a_{2}$ a should be determined primarily by at least a third order perturbation in the crystalline field。 38

\section{Conclusion}

EPR studies of $x$-irradiated single crystals of NaF containing iron as an impurity have been conducted near $4.2^{\circ} \mathrm{K}$ and at temperatures between $77^{\circ} \mathrm{K}$ and $180^{\circ} \mathrm{K}$ 。

Three (EPR) spectra with isotropic $g$ values have been distinguished: One is the well-known $F$ center spectrum: the second is centered near $g=2.002$, and exhibits a partially resolved structure which is anisotropic; the third is centered near $g=4.334$, and corresponds to the $\mathrm{Fe}^{+}$spectrum that was previously determined by Bleaney and Hayes。 25 The first two spectra are readily observable near $77^{\circ} \mathrm{K}_{0}$ while the $\mathrm{Fe}^{+}$ spectrum is observed near $4.2^{\circ} \mathrm{K}$ 。

The observed anisotropy of the second spectrum has been successfully described in terms of a spin Hamiltonian consisting of (i) a Zeeman term for an effective spin $S=5 / 2$, (i1) a fine structure term for an Sostate ion located at the center of a cubic crystalline field and (iii) a hyperfine interaction term pertaining to six equivalent nuclei of spin $1 / 2$ each. The spin Hamiltonian has lead to the identification of the ion responsible for the spectrum to be $\mathrm{Fe}^{3+}\left({ }^{6} \mathrm{~S}_{5 / 2}\right)$ located at a cation site, interacting with the cubic crystalline field 
and with the nearest neighbor fluorine nuclei. The results indicate that the charge-compensating vacancies (two cation-ion vacancies per $\mathrm{Fe}^{3+}$ ) are far from the site of $\mathrm{Fe}^{3+}$ and do not disturb the cubic crystalline field surrounding $\mathrm{Fe}^{3+}$ 。

As the temperature is raised from $77^{\circ} \mathrm{K}$ to $180^{\circ} \mathrm{K}$, the $\mathrm{Fe}^{3+}$ spectrum changes in both its structure and amplitude. Computer analyses of the observed temperature dependence have indicated that the changes are due primarily to the temperature dependence of the fine structure constant (a), and the width $(W)$ of the individual lines composing the $\mathrm{Fe}^{3+}$ spectrum。 The analyses also have ylelded that a decreases almost linearly by approximately $60 \%$ over the temperature range and $\mathrm{W}$ increases as the square root of the temperature.

The results are consistent qualitatively with the existing theories of the S-state ion. Quantitatively, however, the change (60\%) of a from $77^{\circ} \mathrm{K}$ to $180^{\circ} \mathrm{K}$ is just too big to be satisfactorily accounted for by the corresponding change ( $2 \%$ to $3 \%$ ) expected from the theoretical expression for a. Simflar studies on S-state lons are being conducted in order to examine further this point of concern. 
CHAPTER V

IRON IMPURITY CONTROLLED F TO M CONVERSION IN

$$
\text { X-IRRADIATED NaF* }
$$

The EPR investigations described in Chapter IV have shown that iron entering the NaF lattice as a divalent ion can be transformed into $\mathrm{Fe}^{3+}$ or $\mathrm{Fe}^{+}$by $\mathrm{x}$-irradiation. Among other things, the presence of these ions has indicated that these samples have an excess of cation vacancies, which are free to migrate in the lattice. Optical bleaching of these samples can now be compared to samples without the iron impurity。 The results of these studies can be used to determine how the iron impurity affects the optical bleaching mechanism。

\section{Introduction}

The mechansin that governs the optical bleaching has been the subject of several recent papers.39-45 An interesting review of the subject has been given by Compton and Rabin ${ }^{5}$ Two mechanisms appear to be in general use, one proposed by Luty ${ }^{39}$ and the other by Delbecq. ${ }^{41}$ Lity's mechanism consists of the following steps: (1) the F-light dissociates an F center into an a center (anion vacancy) and an electron $\left(e^{-}\right),(2)$ the electron is trapped at another $F$ center to form an $F^{\prime}$ center (a negatively charged $F$ center), and (3) the $\alpha$ center migrates to the $F^{\circ}$ center to form an $M$ center. The three steps are schematically represented as follows:

* The content of this chapter is based on a manuscript submitted to the Physical Review by this author and Y。W。 Kim。 


$$
\begin{aligned}
& \text { (1) } F+h \nu_{F} \rightarrow \alpha+e^{-}, \\
& \text {(2) } F+e^{-} \rightarrow F^{0} \\
& \text { (3) } \alpha+F^{0} \rightarrow M
\end{aligned}
$$

where $h$ and $\nu_{F}$ are Planck's constant and the frequency of the F-light respectively. On the other hand, Delbecq's mechanism differs from Lity ${ }^{\circ} s$ with respect to the second and third steps as follows:

$$
\begin{aligned}
& \text { (1) } F+h \nu_{F} \rightarrow \alpha+e^{-} \\
& \text {(2) } \therefore \alpha+F \rightarrow F_{2}^{-} \\
& \text {(3) } F_{2}^{+}+e^{-} \rightarrow M
\end{aligned}
$$

In other words, the $\alpha$ center formed in step (1) migrates to an F center to form an $\mathrm{F}_{2}^{+}$center (step (2)), which subsequently traps an electron to become an $M$ center. (step (3)).

Neither of the above mentioned mechanisms considers possible Implication of any kind of impurity in the bleaching process. On the other hand, for example, an involvement of anion-cation vacancy pairs in an early stage of optical bleaching of $\mathrm{x}$-1rradiated $\mathrm{KCl}$ has been suggested by Bron and Nowick。 45 However, the nature of the source for the cation vacancies has not been actually understood.

A source of cation vacancies in alkali halide crystals can be provided by a concentration of foreign metallic ions introduced in the crystals. For example, consider iron impurity in NaF. If the Iron impurity is in the triply ionized state $\left(\mathrm{Fe}^{3+}\right)$, for instance, then two cation vacancies per $\mathrm{Fe}^{3+}$ have to be produced in the lattice so that the charge neutrality of the lattice is maintained. Whether or not the two cation vacancies are tightly bound to the $\mathrm{Fe}^{3+}$ to form 
a complex can be determined by investigating the crystalline field of $\mathrm{Fe}^{3+}$ with the method of the electron paramagnetic resonance (EPR) absorption of $\mathrm{Fe}^{3+}$ 。 Furthermore, the EPR absorption of $\mathrm{Fe}^{3+}$ provides information concerning whether $\mathrm{Fe}^{3+}$ is affected by the optical bleachIng of the $F$ centers.

The present work presents the results of an effort to make use of iron impurity as the source of cation vacancies in NaF and to investigate possible involvement of the iron impurity in the optical bleaching of the $\mathrm{F}$ centers in $\mathrm{NaF}$. The formation and site of $\mathrm{Fe}^{3+}$ and $\mathrm{Fe}^{+}$in the lattice of $\mathrm{NaF}$ have been investigated in detall by means of the EPR absorption, in the previous chapter. The results of the EPR work have indicated that the $x$-irradiation transforms the iron impurity from $\mathrm{Fe}^{2+}$ into $\mathrm{Fe}^{+}$and $\mathrm{Fe}^{3+}$. Each of these two lons has been found to be located at a cation site of the lattice with cubic symmetry. The cation vacancies associated with the conversion of $\mathrm{Fe}^{2+}$ to $\mathrm{Fe}^{3+}$ and $\mathrm{Fe}^{+}$ appear to be free to migrate in the lattice rather than being bound to the immediate neighborhood of the sites of the $\mathrm{Fe}^{3+}$ and $\mathrm{Fe}^{+}$ions.

\section{Experimental Procedure and Results}

Crystals of NaF with and without iron impurity were obtained from the Harshaw Chemical Co, Cleveland, Ohio. Samples without iron impurity were also obtained from Optovac, North Brookfield, Massachusetts. Samples about $4 \mathrm{~mm} \times 4 \mathrm{~mm} \times 14 \mathrm{~mm}$ in dimensions were cut with the [110] axis parallel to the longest dimension of each sample. The samples were exposed to $\mathrm{x}$-rays from a Molybdenum target tube operated at $50 \mathrm{kv}$ and $20^{-}$ma for periods of up to 90 hours at room temperature. The irradiated samples were used for both EPR and optical measurements. The optical 
measurements were made at room temperature, with a Bausch and Lomb Spectronic 505 recording spectrophotometer. As mentioned in chapter III, in order to facilitate the optical bleaching experiments, the sample compartment of the spectrophotometer has been modified as illustrated schematically in Fig.7. The bleaching light is a beam of width $20 \mathrm{~m}$, whose central wavelength is equal to $355 \mathrm{~m} \mu$. For the purpose of distinctIon, all samples which exhibit an EPR signal due to $\mathrm{Fe}^{3+}$ will be called Type I samples, and all others will be called Type II samples.

\section{Colorability}

The $F$ center growth of both Type I and Type II samples under $x-$ irradiation has been investigated by treating both types of samples exactly in the same manner. Two typical growth curves are given in Fig, 18, where the intensity of the F-band, which is a measure of the concentration of the $F$ center, is plotted as a function of $x$-irradiation time. To obtain the growth curves, the samples were removed from the $x$-ray unit at several points in time to measure the F-band absorption。 and then they were returned for further irradiation without: exposure to room 1ight. It has been observed that the Type II samples are always colored more readily than the Type I samples in the early stage of coloration. After extended periods (approximately 60 hours or longer) of $x$ irradiation, however, the F center concentrations of both types of samples were approximately equal. These results are rather interesting when compared with recent results of similar studies of the $F$ center formation in $\mathrm{NaCl}$ crystals doped with $\mathrm{Ca}^{2+}$ or $\mathrm{Cd}^{2+}$, which were $\mathrm{x}$-irradiated near room temperature。 46 The $\mathrm{Ca}^{2+}$ impurity appeared to enhance the $\mathrm{F}$ center production, while the $\mathrm{Cd}^{2+}$ impurity apparently had no effect. 


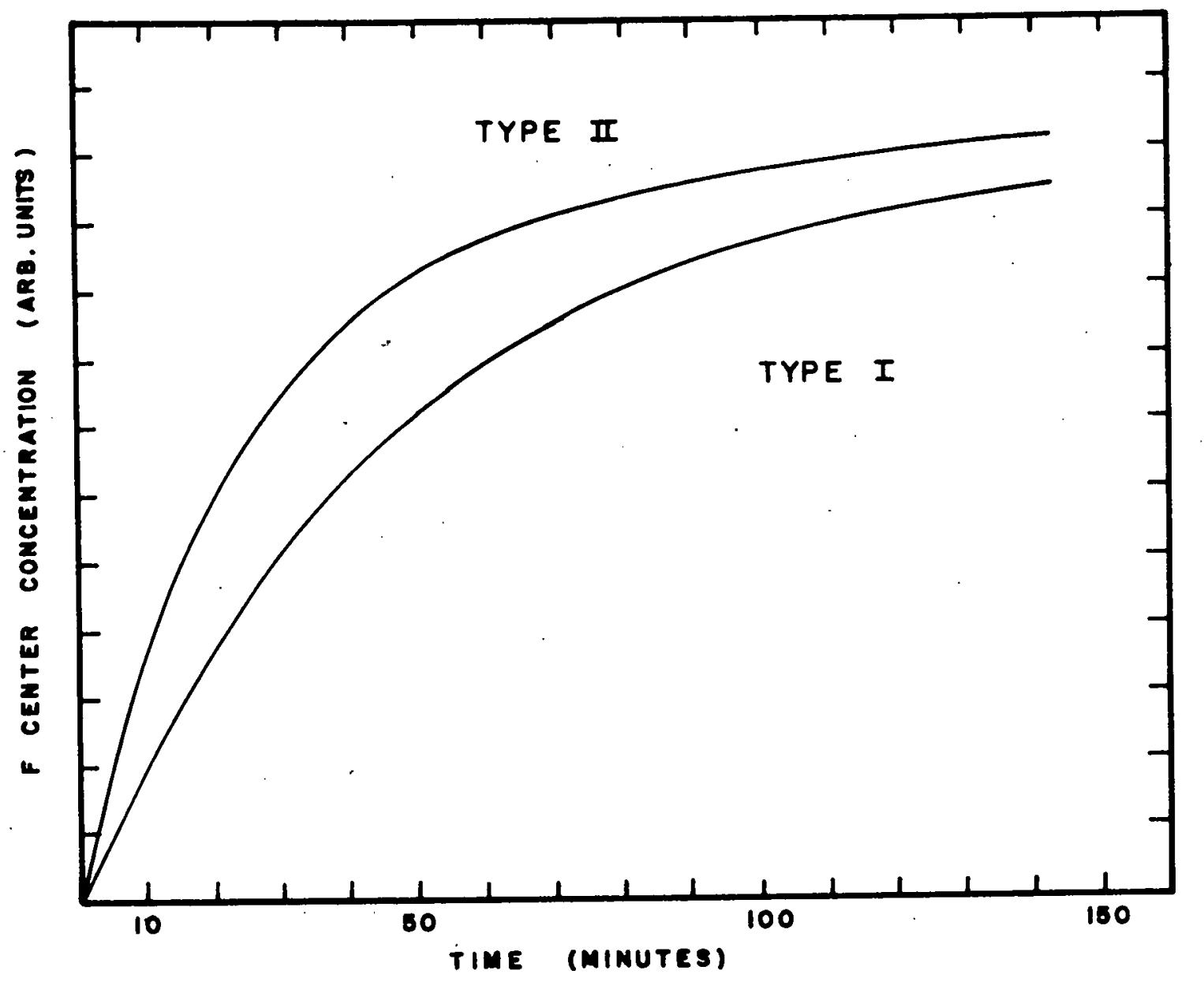

Fig. 18. Typical x-ray colorability curves for $F$ centers in Type I and Type II samples at room temperature. 


\section{Optical Bleaching Efficiency}

Samples of both types, $x$-irradiated for the same interval of time (90 hours), were subsequently optically bleached with F-light at room temperature. As mentioned in Section $I$, the optical bleaching affects both the F-band and the M-band. In Fig, 19, the observed variation of the $F$ and $M$ bands with the controlled bleaching time is illustrated by the two curves labelled $F_{I}$ and $M_{I}$ for a Type $I$ sample, and by the other two curves labelled $F_{I I}$ and $M_{I I}$ for a Type II sample。 The bleaching was interrupted at several points in time to measure the optical absorption spectrum of the samples. The major difference is that the changes in Type II samples occur only after much longer periods of time than the corresponding changes in Type I samples. In other words, the bleaching process is consistently more efficient in the Type I samples. The Type II sample used to obtain the bleaching curves in Fig。 19 showed the poorest bleaching efficiency of all the Type II samples measured. The difference in the bleaching efficiency between the two types of samples has been observed to become more noticeable as the period of $x$-irradiation is increased。

The EPR signals due to $\mathrm{Fe}^{3+}$ and $\mathrm{Fe}^{+}$in Type I samples remain unchanged both in intensity and structure during and after optical bleaching.

\section{Aging Effects}

If the bleaching light is turned off at some time $t=t_{A}$, and the sample is allowed to remain in the dark near room temperature for a certain time period $\Delta_{9}$ three major effects due to the interruption of bleaching are observed in a subsequent bleaching starting from 


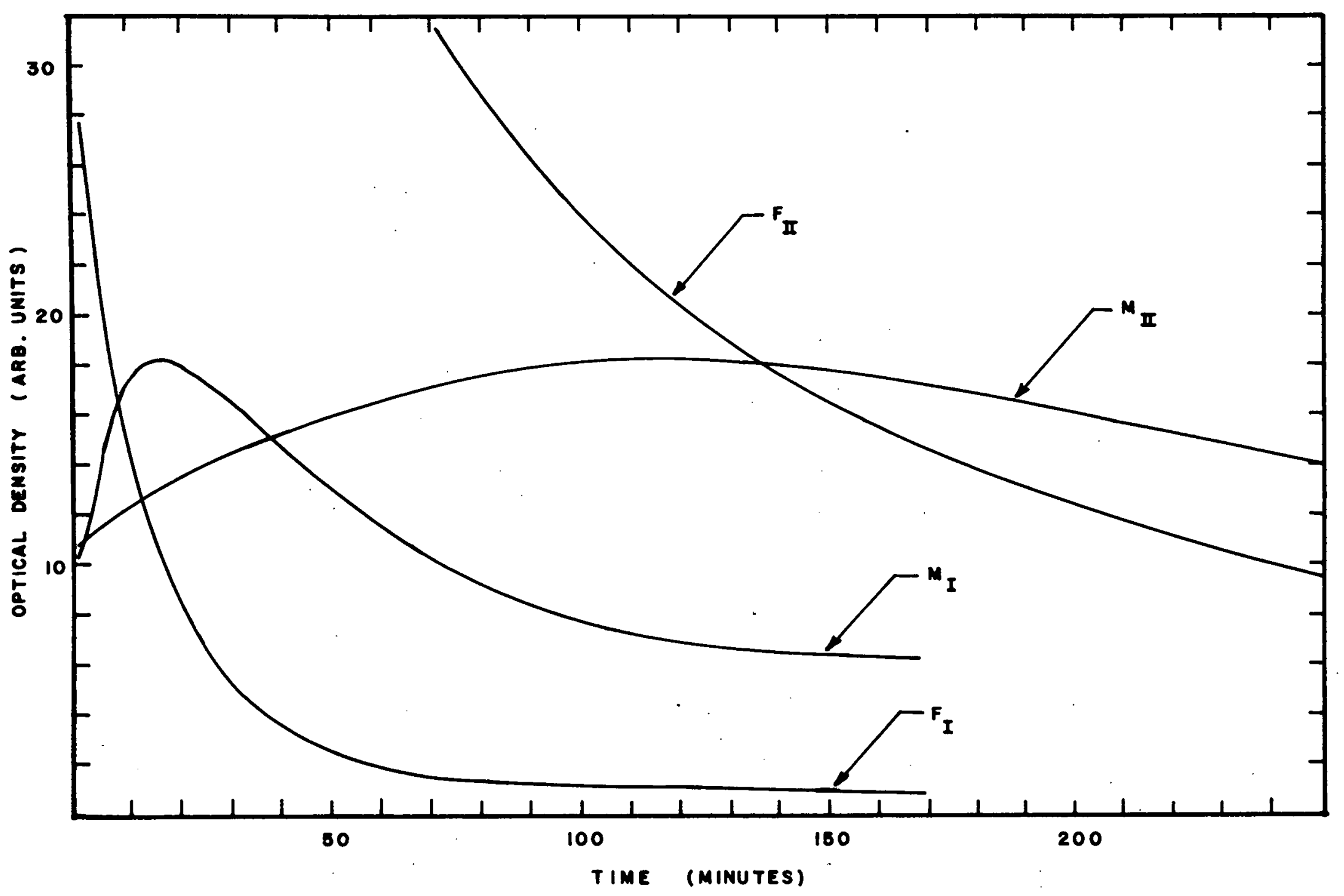

Fig. 19. Typical optical bleaching, curves for the $F$ and $M$ centers in Type $I$ ( $\left(F_{I}\right.$ and $\left.y_{I}\right)$ and Type II ( $F_{I I}$ and $\left.{ }_{I I}\right)$ samples. 
$t=t_{A}+\Delta_{0}$ First, the concentration $n$ of the color center at $t=t_{A}+\Delta$ is the same as that at $t=t_{A^{2}}$ but the rate of bleaching, $-d n / d t$, at time $t_{A}+\Delta$ is substantially larger than that at time $t_{A}$. Secondly, for $t$ larger than $t_{A}+\Delta_{\theta}$. the rate of bleaching and also the concentration of the color center approach rapidly the values that they would have had, if the bleaching had not been interrupted. Third, these effects vary for different choices of $t_{A}$ and $\Delta_{0}$ These "aging" effects are observed for both the $F$ center and the $M$ center.

Figure 20 illustrates the aging effects by means of a typical Mband bleaching curve (optical density of the M-band vs。 the net period of bleaching) of a. Type I sample. At an arbitrarily picked point A for which $t=t_{A}$ o the F-light is turned off and the sample is kept in the dark for $\Delta=382$ minutes. In the figure, $t_{A}$ and $t_{A}+382$ minutes coincide with each other, because of the nature of the time axis mentioned above, and $n$ at $t_{A}$ is found to be the same as that at $t_{A}+382$ minutes。 When the bleaching is resumed at $t_{A}+382$ minutes, $n$ decreases as indicated by the solid curve from point $A$ to point $B_{\text {o }}$ at which point the F-light is turned off again. The rate of bleaching at time $t_{A}+382$ minutes is found to be approximately nine times larger than that at time $t_{A}$ (see next section)。 The dashed curves starting from points $A$ and $C$ represent portions of the bleaching curve which would be expected without the interruption of the original bleaching from $t_{A}$ to $t_{A}+382$ minutes (see next section)。In particular, the dashed curve extending from point $C$ is asymptotic to the solid curve from A to $B$. This seems to indicate that as the resumed bleaching proceeds from $t_{A}+382$ minutes, the values of n and $-\mathrm{dn} / \mathrm{dt}$ rapidly approach the values that they would have had, had there not been the interruption of bleaching at $t_{A}$. 


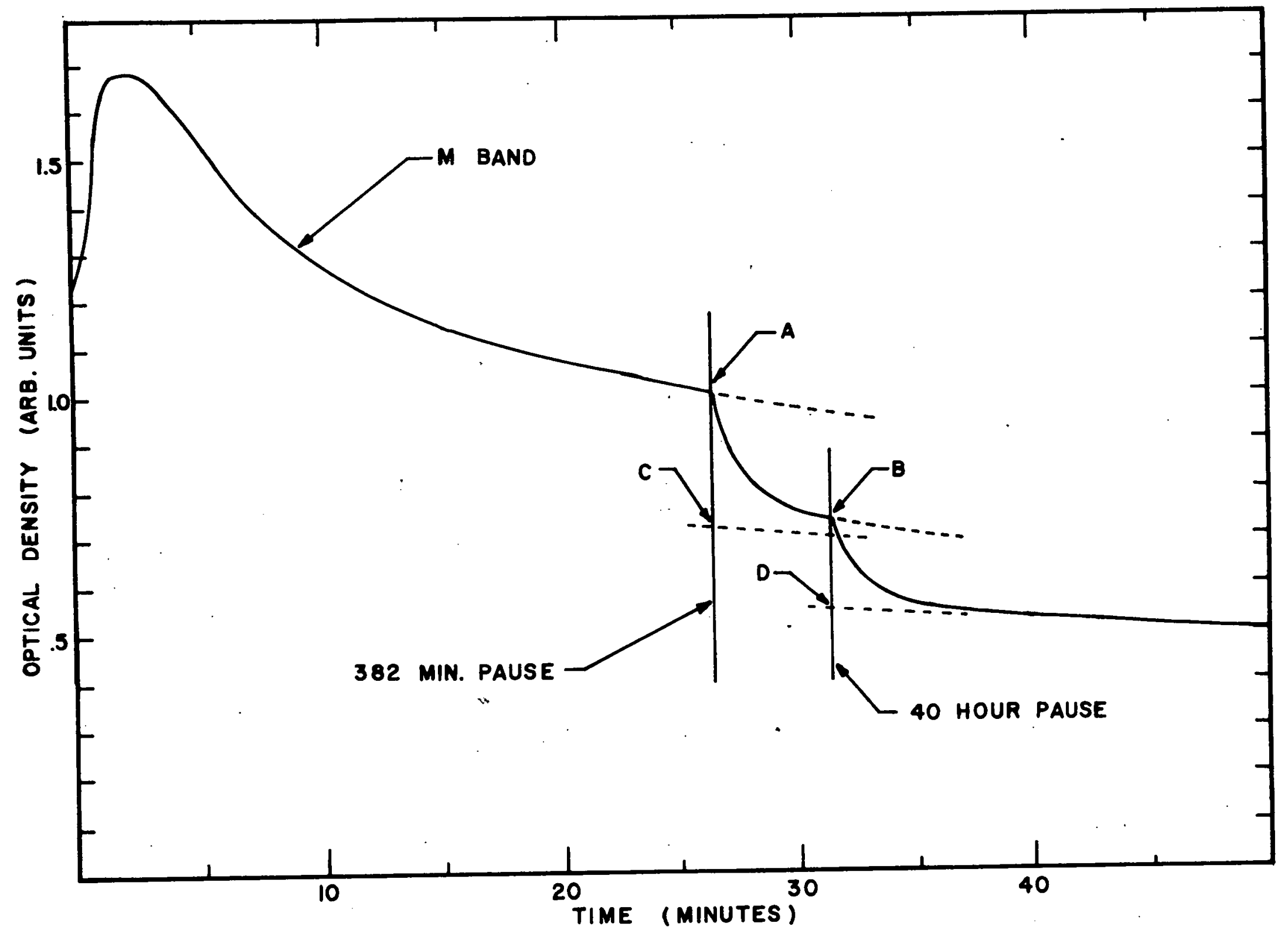

Fig. 20. A typical M center optical bleaching, curve of a Type I sample illustrating aging effects after pauses in the F-light optical bleaching at points $A$ and $B$. The optical density of the $M$ band is plotted as a function of the net bleaching time. As for the dashed curves, see text 
This effect can be observed any number of times. This is illustrated by the portion of the bleaching curve to the right of point $B$ of Fig。 20. The bleaching is terminated at the point $B$ for which $t=t_{B}$, and the sample is kept in the dark for $\Delta=40$ hours, and subsequently the bleaching is resumed at $t=t_{B}+40$ hours. The solid curve to the right of point $B$ in the figure indicates the subsequent bleaching. The dashed curves extending from points $B$ and $D$ represent the continuation of the solid curve between points $A$ and $B_{0}$ which would be obtained without the interruption of the bleaching from $t_{B}$ to $t_{B}+40$ hours。Again, $n$ at $t_{B}$ Is the same as $n$ at $t_{B}+40$ hours, but the rate of bleaching is noticeably enhanced after the second pause in the bleaching. However, the enhanced rate of bleaching is less than that at $t_{A}+382$ minutes, indicating the dependence of the aging effects on the time at which the bleaching is terminated, and also on the value of $\Delta$. Furthermore, the dashed curve from point $D$ is asymptotic to the solid curve from point $B_{0}$ illustrating the behavior of $\mathrm{n}$ and $-\mathrm{dn} / \mathrm{dt}$ (see next section)。

Three more points of significance should be added here. First, the aging effects are far more noticeable with the Type I samples than with the Type II samples. Second, the aging effects are not observable, when the samples are kept at liquid nitrogen temperature during the perfod of bleaching interruption. This, the EPR signals of $\mathrm{Fe}^{3+}$ and $\mathrm{Fe}^{+}$remain unchanged during the bleaching and aging processes。

The observed results pertaining to the aging effects appear to indicate the following: (i) the aging process does not affect the concentration of the color center, nor does it change the concentration of $\mathrm{Fe}^{3+}$ or its cubic crystalline field, (ii) rather, in the process of aging, those defects which are different from the color center and yet 
which are actively involved in the bleaching process are redistributed perhaps through thermally activated migration, and (iii) the majority of these defects are caused by the $\mathrm{Fe}^{3+}$ lons in the lattice, and they are cation vacancies.

\section{Empirical Analysis of the Aging Effects}

The portion of the (solid) bleaching curve of the M-band to the right of the maximum in Fig。 20 can be described very satisfactorily by the following equation

$$
\mathbf{n}=\mathrm{k} /(1+\tau)^{\lambda}
$$

where $\mathrm{n}$ is the concentration of the $M$ centers, $\tau$ is the net period of optical bleaching, and $K$ and $\lambda$ are empirical constants. For the best fit, the values of $\lambda$ have been found to be $0.26,0.085$, and 0.067 for the solid curves between the maximum and point $A$, between point $A$ and point $B$, and to the right of point $B$ in the figure, respectively。

The ratio of the bleaching rate $(-d n / d t)_{+}$immediately after a pause to that $(-d n / d t)$ _ just before the pause can be obtained easily from Eq. (27) as follows:

$$
(-\mathrm{dn} / \mathrm{dt})_{+} /(-\mathrm{dn} / \mathrm{dt})_{-}=\left(\lambda_{+} / \lambda_{-}\right)\left(1+t_{\mathrm{p}}\right)
$$

where $\lambda_{-}$and $\lambda_{+}$are the values of $\lambda$ before and after the pause respectively and $t_{p}$ is the net time period of continuous bleaching up to the pause。

The numerical values of $\lambda_{+}, \lambda_{-}$and $t_{p}$ to be used for points $A$ and $B$ are listed in Table 2。 When these values are introduced into Eq. (28), the ratio of bleaching rates is found to be approximately 9.0 at point $A$ and 4.7 at point $B$ 。 
$-70-$

TABLE 2

THE VALUES OF EMPIRICAL PARAMETERS OF EQ。 (28)

\begin{tabular}{l|l|l}
\hline & Point A & Point B \\
\hline$\lambda_{+}$ & 0.085 & 0.067 \\
$\lambda_{-}$ & 0.26 & 0.085 \\
$t_{p}$ & 26.5 minutes & 5 minutes \\
\hline
\end{tabular}


The observation that $\mathrm{n}$ and $\mathrm{dn} / \mathrm{dt}$ rapidly approach the values they would have had if the bleaching had not been stopped can be stated as a condition on $\lambda$ used in Eq. (27)。 To see this consider a general case where the bleaching is started at time $t=0$, stopped at $t=t_{1}$ and resumed at $t=t_{2}$. Then the period of aging is given by $\Delta=t_{2}-t_{1}$ and

$$
\begin{aligned}
& n=\frac{(n)_{0}}{(1+t)^{\lambda_{1}}},\left(t<t_{1}\right), \\
& n=\frac{k_{2}}{\left(1+t^{\prime}\right)^{\lambda_{2}}},\left(t>t_{2}\right),
\end{aligned}
$$

where

$$
K_{2}=n\left(t_{1}\right)=\frac{(n)_{0}}{\left(1+t_{1}\right)^{\lambda_{1}}}
$$

and $t^{\prime}=t-t_{2}$. In order that $n$ approaches but never becomes less than the value it would have had if the bleaching had been continuous,

$$
\frac{k_{2}}{\left(1+t^{v}\right)^{\lambda_{2}}}-\frac{(n)_{0}}{(1+t)^{\lambda_{1}}} \geqq 0, \quad\left(t>t_{2}\right) \text {. }
$$

This yields the following restriction on the value of $\lambda_{2}$ :

$$
\lambda_{2} \gtrsim \lambda_{1}\left\{1-\frac{\ln \left(t_{1}\right)}{\ln \left(t_{2}\right)}\right\}
$$

This condition is always found to be satisfied experimentally。 In fact, the empirical values of $\lambda_{2}$ have been found to be very close to the largest value permitted by Eq。(32)。

\section{Proposed Mechanism of Optical Bleaching}

The experimental results mentioned in the preceding have established reasonably. well that the $\mathrm{Fe}^{3+}$ and $\mathrm{Fe}^{+}$impurity ions are 
responsible for the optical distinction of the Type I samples from the Type II samples. However, the impurity ions do not participate in the bleaching process directly, that is to say, through thermally activated migration in the lattice, Rather, the cation vacancies associated with the 1mpurity ions seem to migrate and influence the bleaching processes。

Therefore, in order for a mechanism to be responsible for the bleaching processes, it must incorporate these cation vacancies in one way or another. In view of the experimental results, the following mechanism is proposed:

$$
\begin{aligned}
& \text { (1) } F+h \nu_{F}+\alpha+e^{-}, \\
& \text {(2) } \alpha+\tilde{\alpha} \rightarrow(\alpha, \tilde{\alpha}) \\
& \text { (3) }(\alpha, \tilde{\alpha})+F \rightarrow M_{S} \rightarrow F_{2}^{+}+\tilde{\alpha}, \\
& \text { (4) } F_{2}^{+}+e^{-} \rightarrow M
\end{aligned}
$$

Step (1) is the same as the first step of the existing mechanisms mentioned in Section I。 In step (2) the $\alpha$ center couples with one of the cation vacancies $(\tilde{\alpha})$ due to the iron impurity, thereby forming a cationanion vacancy pair $\left(\alpha_{8} \tilde{\alpha}\right)$. In step (3) the migrating vacancy pair $(\alpha, \tilde{\alpha})$ encounters an $F$ center and forms an $M_{S}$ center, which corresponds to the model of the $M$ center originally proposed by Seitz。 ${ }^{1}$ This particular step was previously suggested by Seitz for the formation of the $M$ center。 However, the $M_{S}$ center has never been detected, and it is probably unstable. Therefore, the step (3) continues by the disintegration of the $\mathrm{M}_{\mathrm{s}}$ center into an $\mathrm{F}_{2}{ }^{+}$center and an ã center. Finally (step (4))。 the $\mathrm{F}_{2}{ }^{+}$center captures an electron to form an $M$ center. The $\alpha_{0} \tilde{\alpha}_{\theta}$ $(\alpha, \tilde{\alpha})$ and $M_{s}$ centers are illustrated in Fig。21. A possible mode of the $M_{s}$ center decay is illustrated in Fig。22。 As illustrated, the ions 


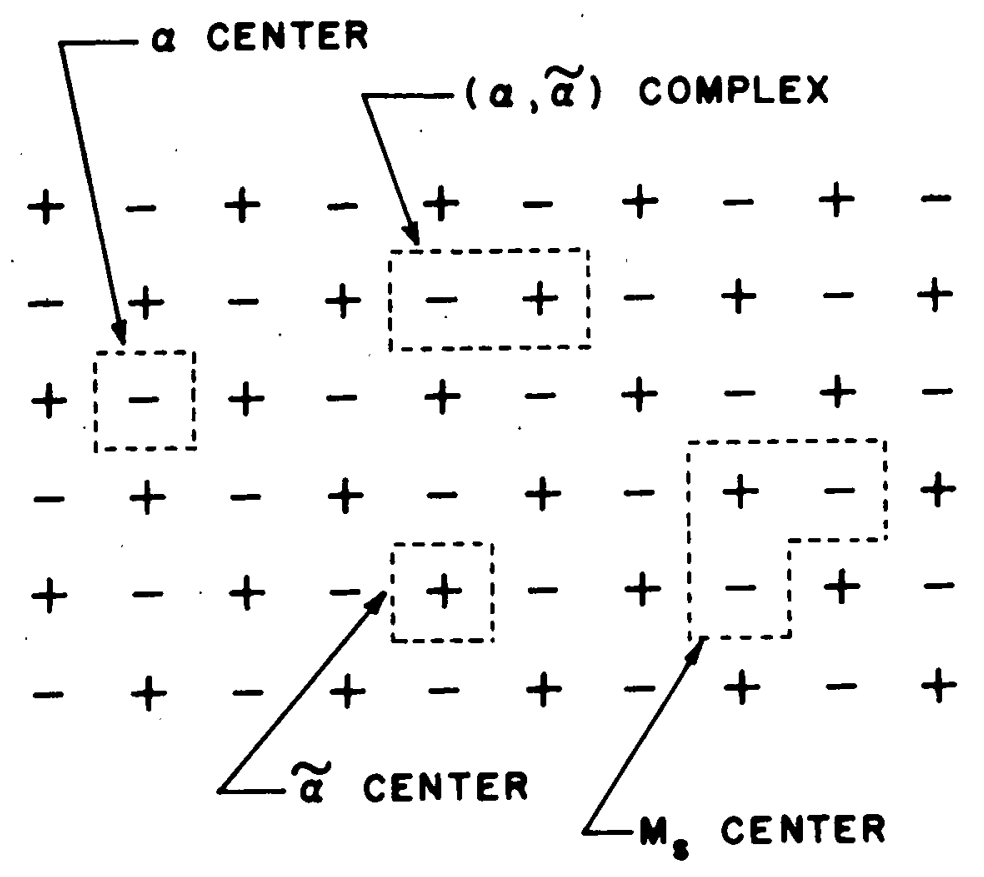

Fig. 21. Schematic diagram 11lustrating the models of an anion vacancy $(\alpha)$, a cation vacancy $(\tilde{\alpha})$, a vacancy pair $(\alpha, \tilde{\alpha})$, and the Seltz model of an $M$ center $\left(M_{s}\right)$. $e^{-}$indicates a trapped electron. 

$\oplus \Theta \oplus \Theta \oplus$
$\oplus \Theta \oplus \Theta \oplus$
$\Theta \oplus \Theta \oplus \Theta$
$\ominus \oplus \Theta+\Theta$
$\oplus-+\Theta \oplus$
$\oplus-\oplus \Theta \oplus$
$\odot \oplus-\oplus \odot$
$\Theta \oplus-\oplus \Theta$
$\oplus \ominus \oplus \oplus \oplus$
$\oplus \Theta \oplus \Theta \oplus$
UNSTABLE
STABLE CONFIGURATION

\section{CONFIGURATION}

Fig. 22. A probable mode of decay. of the $M_{s}$ center into an $M$ center and an $\tilde{\alpha}$ center. $e^{-}$represents a trapped electron. The arrows in the left hand diagram indicate the probable motion of lattice ions. 
surrounding the $\mathrm{M}_{\mathrm{s}}$ center might conceivably move as indicated by the arrows and allow the center to decay.

A few interesting features of the proposed mechanism seem to deserve comments, First, the cation vacancy involved in the steps (2) and (3) is one and the same cation vacancy, which thus acts like a catalyst。 Second, the vacancy pair is expected to be more mobile than isolated vacancies 1,47 This increased mobility of the vacancy pair should contribute to a corresponding increase in the "encounter" probability of the vacancy pair with an F center. Consequently, the sequence of steps (2) and (3) will definitely increase the efficiency of the optical processes as compared with other mechanisms which depend solely on isolated vacancies. Third, the rate of formation of the centers, and hence the $M$ centers, is proportional to the concentration." of the vacancy pairs $(\alpha, \tilde{\alpha})$. But the rate of formation of $(\alpha, \tilde{\alpha})$ is proportional to the concentration of the $\tilde{\alpha}$ centers. Hence, if the concentration of the $\tilde{a}$ centers is large enough, the proposed mechanism will be a dominant one for the formation of the $M$ centers because of the larger mobility of the $\left(\alpha_{0} \tilde{\alpha}\right)$ centers over the isolated $\alpha_{\theta}$ and $\tilde{\alpha}$ centers (see the second comment above)。 If the concentration of the $\tilde{\alpha}$ centers is neglibily small, the dominant mechanism would be one of the mechanfsms discussed earlier (see Intro.), since these mechanisms are expected to be effective concurrently with the mechanism discussed above.

There is no doubt that the proposed mechanism is a very simple one in nature, particularly in view of the fact that there are always many other impurities present in commercial samples of $\mathrm{NaF}$ and other alkali halides。 However, it is interesting, and significant as well, to see how consistently the observed results may be explained in terms of the proposed 
mechanism of such a simple nature. This topic will be taken up in the next section。

\section{Discussion of the Experimental Results}

The proposed mechanism is based on the avallability of excess cation vacancies due to the Iron impurity and their participation in the formation of cation-anion vacancy pairs. The observed distinction between the two types of the samples seems to be consistently explained in terms of the excess cation vacancies due to the iron impurity and the proposed mechanism as summarized below。

\section{Colorability}

The observed lower efficiency in the colorability of the Type I samples relative to the Type II samples can be explained in terms of the $\tilde{\alpha}$ centers which are to be produced in the process of $x$-ray conversion of $\mathrm{Fe}^{2+}$ to $\mathrm{Fe}^{3+}$ and $\mathrm{Fe}^{+}$。 Because of the charge neutrality, each $\mathrm{Fe}^{2+}$ is accompanied with a charge compensating a center. The EPR absorption of $\mathrm{Fe}^{2+}$ is paramagnetic and also its concentration ( $\left.8 \mathrm{ppm}\right)$ is more than enough to yield an observable EPR signal. This is probably due to the $\tilde{\alpha}$ center which is fairly tightly bound to the site of the $\mathrm{Fe}^{2+}$ Ion. This a center, however, may be made to migrate, 29 when $\mathrm{Fe}^{2+}$ is transformed into $\mathrm{Fe}^{+}$. This indicates a physical separation of the $\tilde{a}$ center from the iron impurity site. Furthermore, the conversion of $\mathrm{Fe}^{2+}$ to $\mathrm{Fe}^{3+}$ contributes additionally two $\alpha$ centers per $\mathrm{Fe}^{3+}$ formed。Therefore, the Type I samples subjected to the $x$-irradiation may contain more $\tilde{\alpha}$ centers than the similarly treated Type II samples. During $x$-irradiation these excess a centers combine with some of the a centers produced by the $x$ irradiation. These vacancy pairs migrate and enhance the 
recombination of vacancies and interstitials during $x$-irradiation。 Therefore, the number of a centers available for the formation of the F centers is definitely less in the Type I samples than in the Type II samples. In other words, the Type I samples are less easily colored than the Type II samples.

\section{Bleaching Efficiency}

The existence of excess $\tilde{a}$ centers due to the $x$-ray conversion of the iron impurity mentioned in the preceding can activate the steps (2) and (3) of the proposed mechanism in the previous section, thus contributing to the observed enhancement of the bleaching efficiency of the Type I samples over the Type II samples, for which Delbecq ${ }^{\circ}$ s mechanism 1s probably dominant.

Since the $\tilde{\alpha}$ centers are produced gradually under $x$-irradiation the difference in optical bleaching efficiencies between Type I and Type II samples would be expected to become more noticeable for a longer x-irradiation time. This actually has been observed:in the present experiment。

\section{Aging Effects}

Before bleaching commences, the excess $\tilde{\alpha}$ centers and $\mathrm{F}$ centers are presumably distributed randomly through the lattice. Some F centers will have an $\tilde{\alpha}$ center nearby and others will not. During bleaching, $\left(\alpha_{0} \tilde{\alpha}\right)$ centers have a better chance of beling formed, if an $F$ center, which is the source of an $\alpha$ center during bleaching, and an $\alpha$ center are close to one another。 Once formed, the $(\alpha, \hat{\bar{\alpha}})$ center, with its relatively high mobility, can migrate to form an $M$ center according to the steps (2)。 (3), and (4). As the bleaching proceeds, the average distance between 
an $F$ center and an $\tilde{\alpha}$ center tends to increase and consequently the probabllity of formation of $(\alpha, \tilde{\alpha})$ centers tends to decrease。 However, when the sample is allowed to remain in the dark at room temperature, the a centers, which are still able to migrate, may redistribute themselves。 In doing so many $F$ centers will acquire an a center in their immediate neighborhood。Thus, when bleaching starts again, the bleaching rate will be higher than when the bleaching was stopped. This accounts for the aging effects。

\section{Conclusion}

Effects of iron Impurity on the x-ray colorability and the subsequent optical bleaching of $\mathrm{NaF}$ have been investigated near room temperature. EPR and optical absorption measurements have been made on NaF samples.with (Type I) and without (Type II) iron impurity. The results have Indicated that the colorability of Type I samples 1s poorer than that of Type II samples, but the efficiency of the optical bleaching of Type I samples is significantly larger than that of the Type II samples。

The optical bleaching has been found to exhibit "aging" effects。 The aging effects are far more noticeable with Type I samples than with Type II samples。

In order to account for the observed distinction between the two types of samples, an impurity controlled mechanism of optical bleaching 1s proposed. The mechanism is based on the avallability of excess cation vacancies associated with the iron Impurity, and their participation in the formation of cation-anton vacancy pairs。 
CHAPTER VI

OBSERVATION OF AN UNSTABLE N-BAND IN NaF

In several alkali halides, when optical bleaching is continued for extended periods, optical absorption bands due to $\mathrm{N}$ centers (see Chapter II and Fig。3) appear on the long wavelength side of the F-band. In NaF no prominent $\mathrm{N}$-bands have been observed. In this chapter experimental evidence of an unstable $\mathrm{Nab}$ and in $\mathrm{x}$-irradiated $\mathrm{NaF}$ is presented. A summary of this work has recently been published by this author and Y.W. Kim in the Physics Letters. 48

In the case of $\mathrm{LiF}, \mathrm{KCl}, \mathrm{KBr}, \mathrm{NaCl}$, and $\mathrm{KI}$, the $\mathrm{N}$-bands have been observed, 2,49-51 and they seem to satisfy an Ivey type relation: 52

$$
\begin{aligned}
& \lambda_{\max }=1730 \mathrm{~d}^{1.52} \text { for the } \mathrm{N}_{1} \text {-band, } \\
& \lambda_{\max }=1880 \mathrm{~d}^{1.52} \text { for the } \mathrm{N}_{2} \text {-band, }
\end{aligned}
$$

where $\lambda_{\max }$ and $d$ are the wavelength of the absorption maximum of the band and the lattice constant, respectively, both in Angstrom units. The two empirical Ivey relations for the N-bands are illustrated by the two straight lines labelled as $\mathrm{N}_{1}$ and $\mathrm{N}_{2}$ in Fig。23。. It should be noted here that the $\mathrm{N}_{1}$ - and $\mathrm{N}_{2}$-lines in $\mathrm{Fig} 。 23$ are drawn without any attention to the experimental point for NaF to be described in this chapter.

The band investigated here (simply band $\mathrm{N}$ for convenience) has an absorption maximum at approximately $727 \mathrm{m \mu}(1.705 \mathrm{ev})$ and a half width of approximately $0.142 \mathrm{ev}$, and is unstable at $300^{\circ} \mathrm{K}$. It can be produced in single crystals of $\mathrm{NaF}_{\text {, }}$ with varylng degrees of difficulty, depending upon the Ingots of Harshaw and Optovac。

The Type I samples, whlch were referred to in Chapter $V$ as having a 


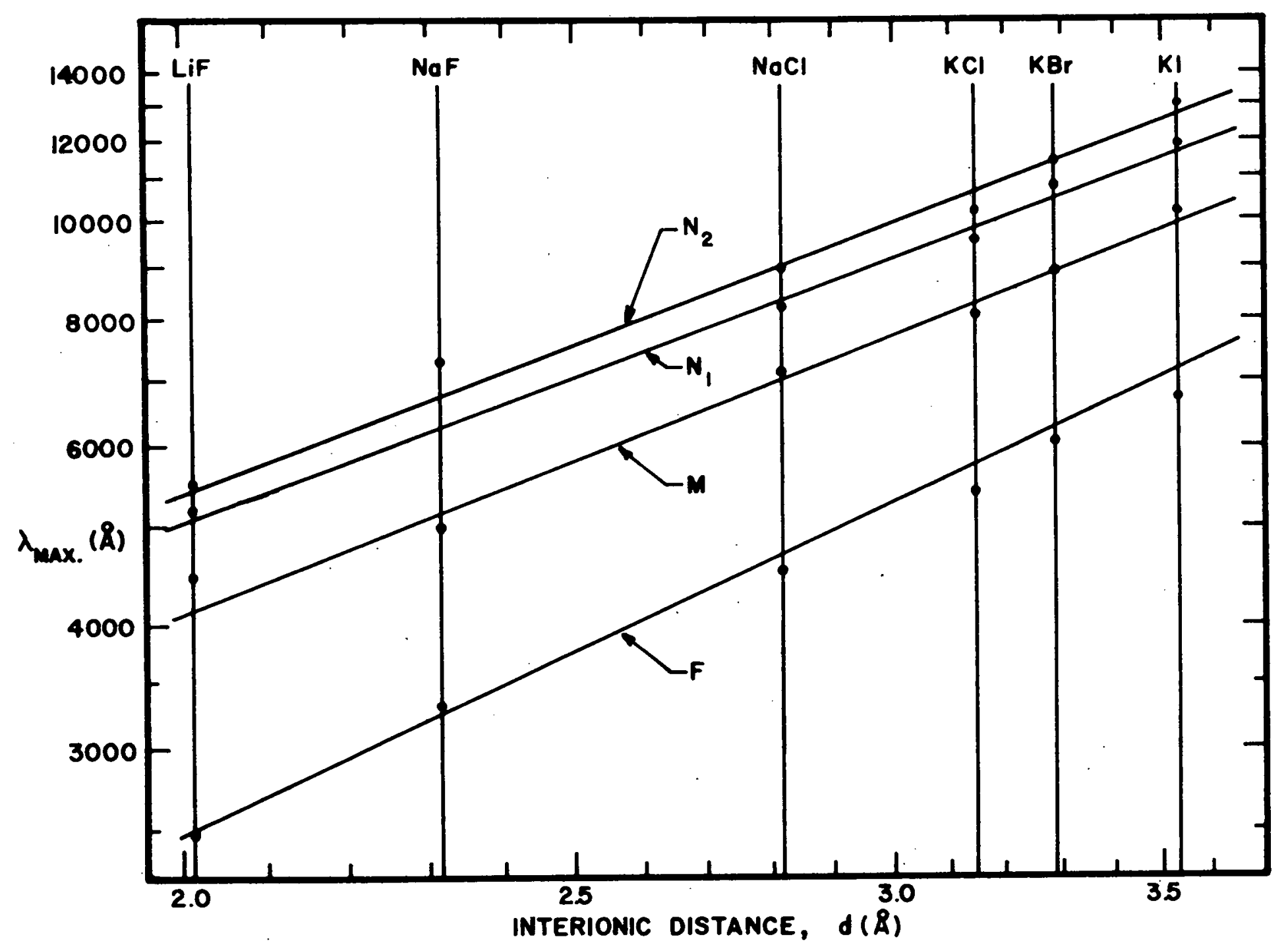

Fig. 23. $\lambda$ (dots) of $N$ bands, and also $F$ and $M$ bands, observed in several alkali halides, and Ivey's relations (straight lines) for the bands. The straight lines for the $F$ and $M$ bands are taken from ref. 2: $\lambda_{\mathrm{m}}=703 \mathrm{~d} 1.84$ ( $F$ band) and $\lambda_{\max }=1400 \mathrm{~d} 1.56$ ( $M$ band). They are included here for comparison. 
high bleaching efficiency, also developed the most intense band $N_{0}$ This Is not unexpected since band $\mathrm{N}$ is unstable and the larger the rate of formation, the larger the maximum intensity of the band No This may indeed be the reason why this band has not been observed previously in $\mathrm{NaF}$, since the observation depends on a sample with impurities which enhance the bleaching process。

Typically, a sample of NaF obtained from Harshaw is first Irradlated for 34 hours by x-rays from a Molybdenum target tube operated at $50 \mathrm{kev}$ and $20 \mathrm{ma}$. The 1rradiated sample contains a large concentration of F centers. Subsequently, the F-band centered at $341 \mathrm{~m} \mu$ is bleached with the F-light filtered from a 150 watt high pressure Hg lampo This Fbleach leads first to a very rapid growth of the M-band centered at $507 \mathrm{m \mu}$ to a maximum intensity, after which the M-band decreases. In the process, the band $\mathrm{N}$ continues to grow at a slower rate, and reaches a maximum intensity much later than the M-band. The observed behavior of the Fand $M-b a n d s$, and the band $N$ during the bleaching is 11lustrated in Fig。24。 Notice that the bleaching time is on a logarithmic scale since changes are relatively slow in the region where the band $N$ reaches its maximum。 If, at the point where the band $N$ is at maximum, the bleaching 1 ight 1 s turned off and the sample is allowed to set in the dark, the band $\mathrm{N}$ decays exponentially with a time constant $\tau=7.8$ hours, as illustrated in Fig。 25 .

The band $\mathrm{N}$ of the present work seems to have the following three major simflarities with the known $\mathrm{N}$ bands of $\mathrm{KCl}$ and other alkall halides: (1) The $\lambda_{\max }$ of the band $N$ is fairly close to that predicted by the Ivey relation for the $\mathrm{N}_{2}$ band of $\mathrm{KCl}$ and other alkali halldes (see FIg。23)。 Since $\lambda_{\max }$ of the band $\mathrm{N}$ is obtained at $300^{\circ} \mathrm{K}$, while $\lambda_{\max }$ for the other alkall halides except for LiF in $\mathrm{FIg}$. 23 refers to $77^{\circ} \mathrm{K}$, a temperature 


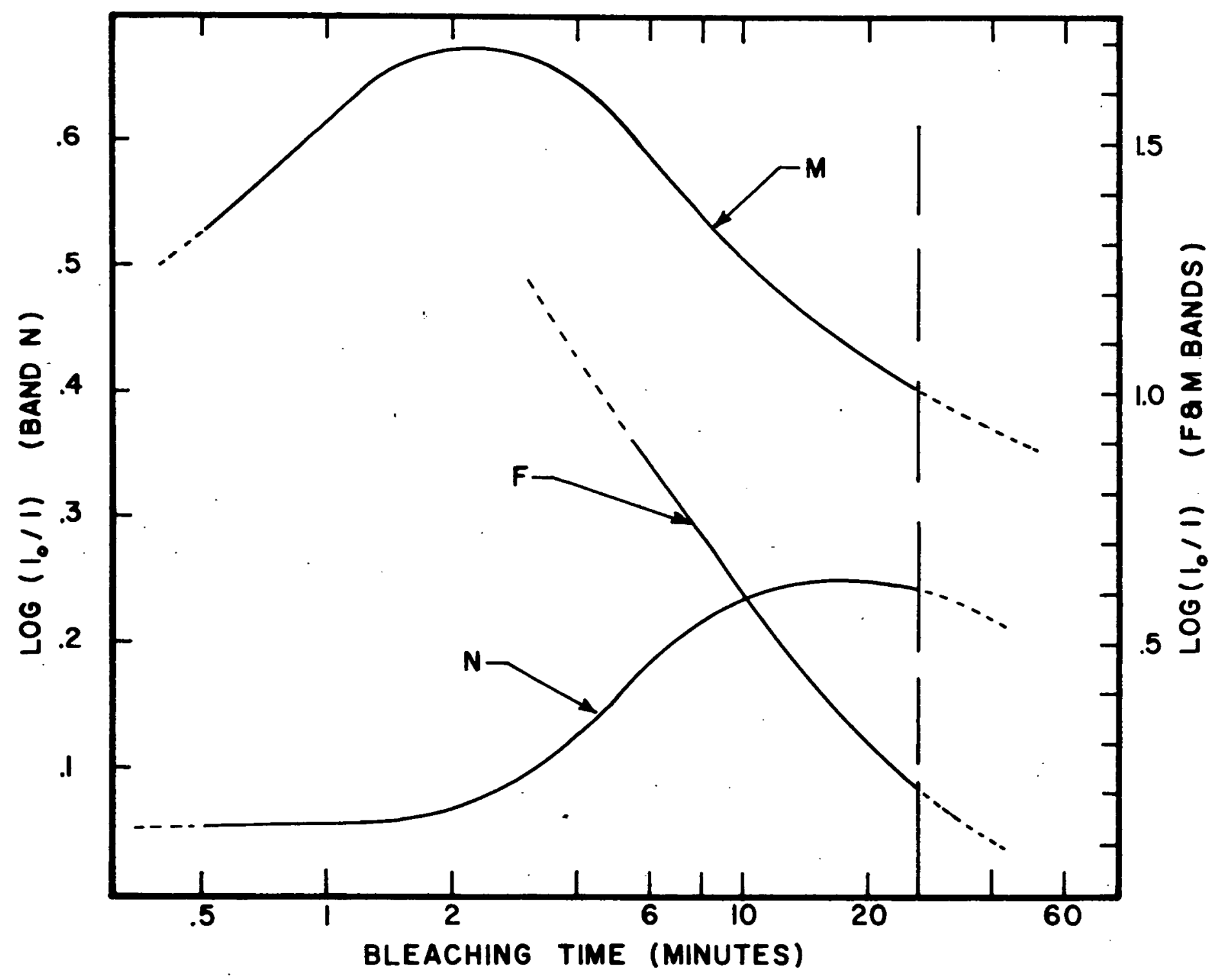

Fig. 24. Behavior of the band $\mathrm{N}$, and the $\mathrm{F}$ and $Y$ bands during optical bleaching with F-light. Note the different ordinate scales for the $F$ and $M$ bands and the band $N$. 


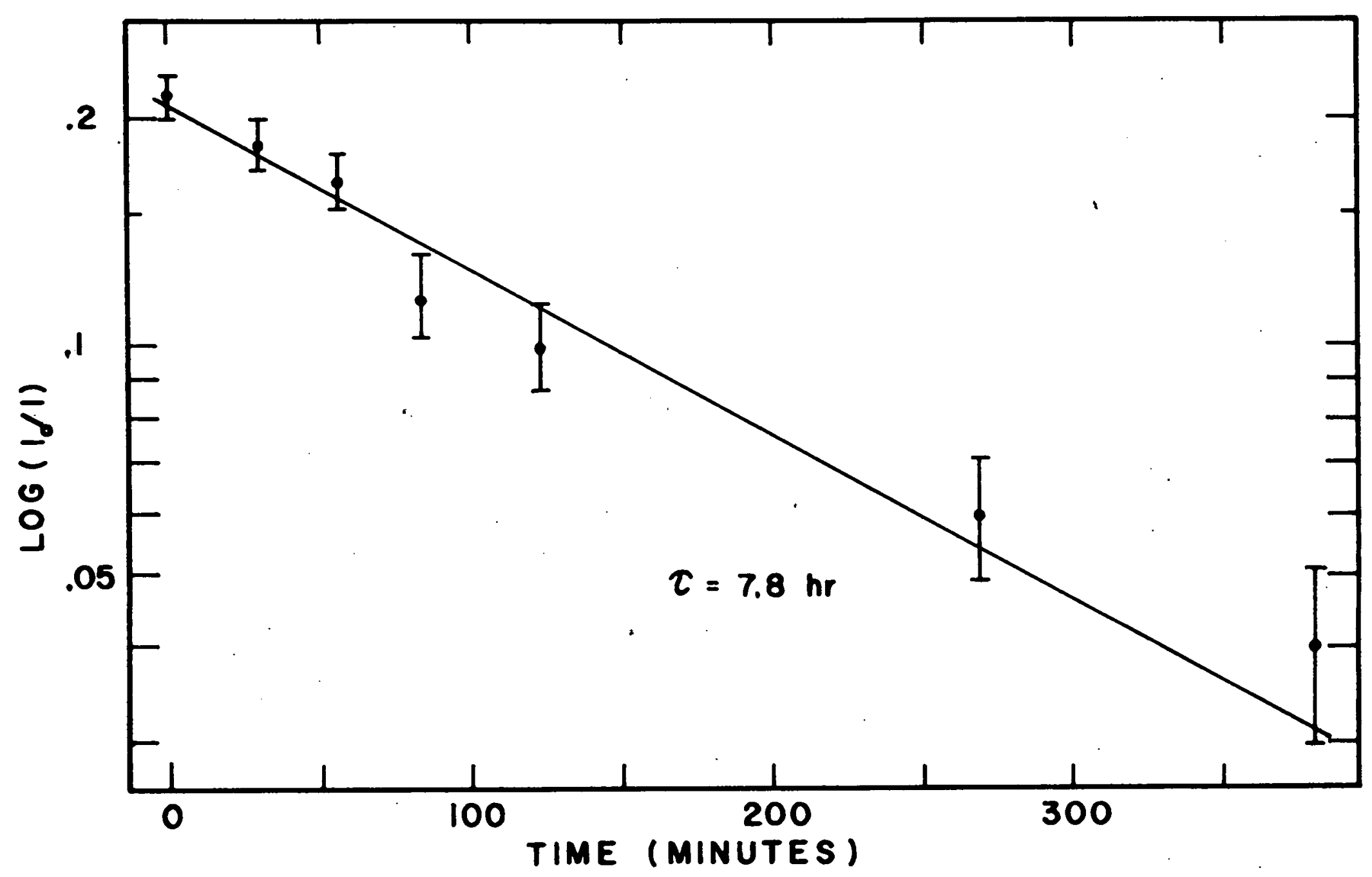

Fig. 25. Exponential decay with time constant 7.8 hours of the band $N$, when left in the dark. 
correction should move $\lambda_{\max }$ of $\mathrm{NaF}$ closer to the $\mathrm{N}_{2}$ line of the Ivey relation by approximately $10 \mathrm{m \mu}$. (2) The formation process of the band. $\mathrm{N}$ by the F-bleach is similar to that of the $\mathrm{N}$ bands in $\mathrm{x}$-irradiated $\mathrm{KC1} .53$ (3) According to Plerce, 54 certain zero-phonon lines appear in the general region of the $\mathrm{N}$ bands of alkall halides. In the case of NaF, four zerophonon lines have been detected. One of them 1ocated at $821.7 \mathrm{~m} \mu$ corresponds to the long wavelength edge of the band $N$ reported here. These simflarities suggest very strongly that the unstable band $N$ observed in the present work is one of the $\mathrm{N}$ bands of $\mathrm{NaF}$, probably the $\mathrm{N}_{2}$ band。 


\section{APPENDIX I}

\section{HYPERFINE INTERACTION TERM IN A SPIN HAMILTONIAN}

The hyperfine term in the spin Hamiltonian for a paramagnetic ion describes the Interaction between the magnetic dipole moment of the paramagnetic ion and ail the neighboring nuclear magnetic moments 55 For simplicity, consider an interaction of the paramagnetic ion with only one nuclear magnetic moment. (For the more general case of many nuclear magnetic moments, it is only necessary to add the interactions due to each of them.) Let

$$
\begin{aligned}
\vec{\mu}_{\hat{s}} & =\text { spin magnetic moment of the paramagnetic ion, } \\
& =-g \vec{B} \vec{S} \\
\vec{\mu}_{\hat{n}} & =\text { magnetic moment of the nucleus, } \\
& =g_{N} \vec{B}_{N} \vec{I}
\end{aligned}
$$

where $B, B_{N}=$ electronic and nuclear Bohr magnetons, respectively,

$$
\begin{aligned}
g, B_{N} & =\text { electronic and nuclear g-factors, respectively, } \\
\vec{S} & =\text { spin of the paramagnetic ion; and } \\
\vec{I} & =\text { spln of the interacting nucleus. }
\end{aligned}
$$

Also let $\ddot{\mathbf{r}}$ be a râdius vêctor in a cartestan coordinate system whose origin coincides with the site of the interacting nucleus.

The Hamiltoñan which describes the manetic effects of this system of a parämagnetic iôn ând one intêracting nuclear mâgnetic moment can be given by

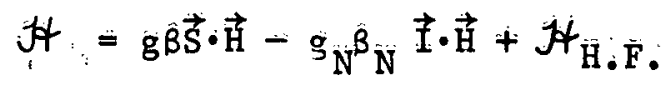

The first termi is the Zeeman energy of the paramagnetic ion and the second term is the nuclear Zeeman energy. The third term is the 
hyperfine interaction term which can be expressed as follows.

$$
I_{H . F .}=\left\{\frac{-3\left(\vec{\mu}_{N} \cdot \vec{r}\right)\left(\vec{u}_{S} \cdot \vec{r}\right)}{r^{5}}+\frac{\vec{\mu}_{N} \cdot \vec{\mu}_{S}}{r^{3}}\right\}-\frac{8 \pi}{3} \vec{u}_{N} \cdot \vec{u}_{S} \delta(\vec{r})
$$

where the first term is the dipole-dipole interaction and the second term is the Fermi contact interaction.

Let $\psi$ be the orbital wave function of the paramagnetic ion. It is now possible to calculate the spin Hamiltonian term for the hyperfine Interaction which is given by

$$
\text { It }_{S, H . F_{Q}}=\int \psi^{*} H_{\mathrm{H}, \mathrm{F} .} \psi \mathrm{d} \tau \text {, }
$$

where the integration is over spatial coordinates only. Hence, using Eqs. $(A-1),(A-2),(A-4)$, and $(A-5)$,

$$
\text { J }_{\mathrm{H}, \mathrm{F} .}=\operatorname{gg}_{\mathrm{N}} \beta B_{\mathrm{N}}\left\{\frac{3(\vec{I} \cdot \overrightarrow{\mathrm{r}})(\vec{S} \cdot \overrightarrow{\mathrm{r}})}{\mathrm{r}^{5}}-\frac{\vec{I} \cdot \overrightarrow{\mathrm{S}}}{\mathrm{r}^{3}}+\frac{8 \pi}{3} \overrightarrow{\mathrm{I}} \cdot \overrightarrow{\mathrm{S}} \delta(\overrightarrow{\mathrm{r}})\right\} \text {. }
$$

and

$J \psi_{S, H \cdot F \cdot}=g g_{N} \beta \beta_{N} \rho\left\{\frac{3(\vec{I} \cdot \vec{r})(\vec{S} \cdot \vec{r})}{r^{5}}-\frac{\vec{I} \cdot \vec{S}}{r^{3}}+\frac{8 \pi}{3} \vec{I} \cdot J \delta(\vec{r})\right\}|\psi(\vec{r})|^{2} d \tau \quad .(A-7)$

Equation (A-7) can be written as

$$
\Psi_{S, H . F .}=\vec{I} \cdot \underline{A} \cdot \vec{S} \text {, }
$$

where $\underline{A}$ is the hyperfine interaction tensor. For convenience $\underline{A}$ is divided into what is called the anisotropic hyperfine tensor $\underline{B}$ and the isotropic hyperfine term $\delta$, as follows:

$$
\underline{A}=\underline{B}+\delta,
$$

The cartesian components of $\underline{B}$ are indicated by $B_{m, n}$ where $m, n=1,2$, or 3 ; and 


$$
\begin{aligned}
& B_{m, n}=g_{N} \beta B_{N} \delta\left\{\frac{3 x_{m} x_{n}}{r^{5}}-\frac{\delta_{m n}}{r^{3}}\right\}|\psi(\vec{r})|^{2} \mathrm{~d} \tau, \\
& \delta \quad=\frac{8 \pi}{3} g_{N} \beta B_{N} \delta \delta(\vec{r})|\psi(\vec{r})|^{2} \mathrm{~d} \tau,
\end{aligned}
$$

In these expressions, $x_{i}$ refers to a component of the vector $\vec{r}_{0}$ At this point it can be shown that the tensor $B$ is traceless.

Trace $\underline{B}=\sum_{i=1}^{3} B_{i i}=g g_{N} \beta \beta_{N} \int\left\{\frac{3\left(x_{1}{ }^{2}+x_{2}{ }^{2}+x_{3}{ }^{2}\right)}{r^{3}}-\frac{3}{r^{3}}\right\}|\psi(\vec{r})|^{2} d \tau=0 \cdot(A-11)$

In order to evaluate the components of $A$, assume that the wave function of the paramagnetic ion $\psi(\vec{r})$ has axial symmetry about the $z$-axis.* The same results can be derived if the $z$-axis is an $n$-fold axis of symmetry where $n \stackrel{3}{=} 3 .^{3}$ with this assumption,

$$
B_{x y}=3 g_{N} B B_{N}\left\{\int_{0}^{\infty} \int_{0}^{\pi} \frac{\sin ^{2} \xi}{r^{3}}|\psi|^{2} r^{2} \sin \xi d r d \xi\right\}\left\{\int_{0}^{2 \pi} \sin n \cos n d n\right\}=0
$$

where $x_{1}=r \sin \xi \cos n, x_{2}=r \sin \xi \sin n$, and $x_{3}=r \cos \xi ;$ and $\xi$ and $n$ are the polar angles of $\vec{r}$ using the line joining the paramagnetic ion and the interacting nucleus as the polar axis (see Fig. 26)。 Likewise

$$
B_{x z}=B_{y z}=B_{y x}=B_{z x}=B_{z y}=0
$$

In a similar manner,

* In what follows the line joining the paramagnetic ton and the nuclear site will be referred to as the z-axis. 


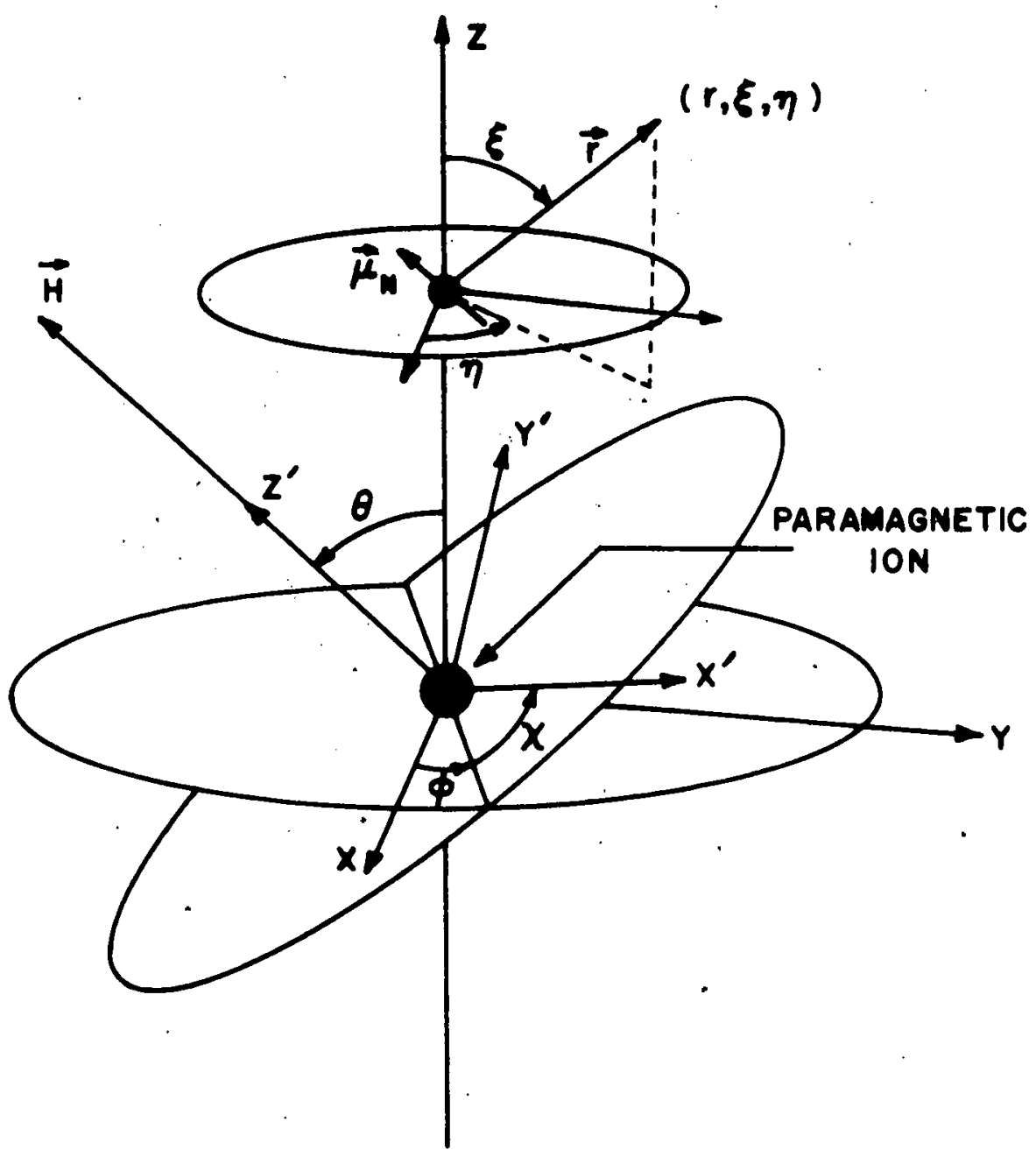

Fig. 26. Coordinate systems used to find the hyperfine Interaction between a paramagnetic lon and a single nuclear magnetic moment. $\mathrm{f}$ is directed along the $z$ ' axis and the nuclear magnetic moment is located on the 2 axis. 


$$
\begin{aligned}
B_{x x} & =g g_{N} B B_{N} \int_{0}^{\infty} \int_{0}^{\pi} \int_{0}^{2 \pi} \frac{3 \sin ^{2} \xi \cos ^{2} n-1}{r^{3}}|\psi|^{2} r^{2} \sin \xi d r d \xi d n \\
& =g g_{N} B B_{N} \int_{0}^{\infty} \int_{0}^{\pi} \frac{3 \pi \sin ^{2} \xi-2 \pi}{r^{3}}|\psi|^{2} r^{2} \sin \xi d r d \xi \\
& =g g_{N} B B_{N} \cdot \int_{0}^{\infty} \int_{0}^{\pi} \frac{3-3 \cos ^{2} \xi-2}{r^{3}} \pi|\psi|^{2} r^{2} \sin \xi d r d \xi \\
& =-g g_{N} B B_{N} \pi \int_{0}^{\infty} \int_{0}^{\pi} \frac{3 \cos ^{2} \xi-1}{r^{3}}|\psi|^{2} r^{2} \sin \xi d r d \xi \\
& =-\gamma
\end{aligned}
$$

also

$$
B_{y y}=-\gamma
$$

and

$$
B_{z z}=g g_{N} B B_{N} \int_{0}^{\infty} \int_{0}^{\pi} \delta_{0}^{2 \pi} \frac{3 \cos ^{2} \xi-1}{r^{3}}|\psi|^{2} r^{2} \sin \xi d r d \xi d n=2 \gamma \quad .
$$

Thus

$$
\underline{A}=\underline{B}+\delta=\left(\begin{array}{ccc}
\delta-\gamma & 0 & 0 \\
0 & \delta-\gamma & 0 \\
0 & 0 & \delta+2 \gamma
\end{array}\right)
$$

referred to the $(x, y, \dot{z})$ system (see Fig, 26) and

$$
I_{S_{,} H_{0} F_{0}}=I_{z} S_{z}(\delta+2 \gamma)+(\delta-\gamma)\left(I_{x} S_{x}+I_{y} S_{y}\right)
$$

Consider the paramagnetic ion and the interacting nuclei to be in a large external magnetic field $\vec{H}$. The electronic Zeeman term is then much larger than the hyperfine interaction term。 In this case $\vec{S}$ is quantized along the magnetic field direction. However, the interacting nucleus sees not only the external magnetic field, but the dipole field due to the magnetic moment of the paramagnetic ion. The nuclear magnetic moment will be quantized along the direction of the external magnetic field in two cases: (1) If the nuclear Zeeman term $\left(g_{N} \vec{N} \cdot \vec{I}\right)$ is much 
larger than the hyperfine interaction and (2) If the isotropic part of the hyperfine interaction (measured by $\delta$ ) is much larger than both the nuclear Zeeman term and the anisotropic hyperfine interaction (measured by B). In the second case the dominant term as far as the nucleus is concerned, is the isotropic hyperfine term which has the form $\delta \vec{S} \circ \vec{I}$ 。 Since in this case $\vec{S}$ is quantized along the direction of $\vec{H}_{2} \vec{I}$ will be quantized along the same direction. The two cases mentioned above describe most of the situations which normally occur. Hence, in what follows, it will be assumed that $\vec{I}$ is quantized along the direction of $\overrightarrow{\mathrm{H}}$ just as is $\overrightarrow{\mathrm{S}}$.

Define an $(x, y, z)$ coordinate system with its origin at the site of the paramagnetic ion and the interacting nucleus lying on the $z$ axis as shown in Fig。26. Also define an $\left(x^{\circ}, y^{0}, z^{\circ}\right)$ system which has Eulerian angles $(\theta, \phi, x)$ relative to the $(x, y, z)$ system。 Let the magnetic field $\overrightarrow{\mathrm{H}}$ be directed along the $z^{\circ}$ axis。 Then $\overrightarrow{\mathrm{S}}$ and $\overrightarrow{\mathrm{I}}$ are quantized along the $z^{\circ}$ axis and the matrix elements of $\vec{I}$ and $\vec{S}$ are known in the $\left(x^{8}, y^{\circ}, z^{\circ}\right)$ system (i。e. $\left.\left\langle I_{x^{p}}\right\rangle=\left\langle I_{y^{p}}\right\rangle=\left\langle S_{x^{\prime}}\right\rangle=\left\langle S_{y^{p}}\right\rangle=0\right)$ ) However, measurements. are made with respect to the $(x, y, z)$ system。' Therefore, Eq. (A-18) must be transformed to the $(x, y, z)$ system, Referring to Fig。 26:

$$
\begin{aligned}
& I_{z}=I_{z^{0}} \cos \theta+\left(\text { terms in } I_{x^{0}}, I_{y^{0}}\right) \\
& I_{x}=I_{z^{0}} \sin \theta \cos \psi+\left(\text { terms in } I_{x^{\prime}}, I_{y^{\theta}}\right) \\
& I_{y}=I_{z} \cdot \sin \theta \sin \psi+\left(\text { terms in } I_{x}, I_{y}{ }^{\prime}\right)
\end{aligned}
$$

and

$$
\begin{aligned}
& S_{z}=S_{z^{\circ}} \cos \theta+\left(\text { terms in } S_{x^{\circ},} S_{y^{\circ}}\right) \\
& S_{x}=S_{z^{\circ}} \sin \theta \cos \psi\left(\text { terms in } S_{x^{\circ},} S_{y^{\circ}}\right) \\
& S_{y}=S_{z^{\circ}} \sin \theta \sin \psi\left(\text { terms in } S_{x^{\circ},}, S_{y^{\circ}}\right)
\end{aligned}
$$


$-91-$

Using Eqs. $(A-18),(A-19)$, and $(A-20)$,

$$
\begin{aligned}
I_{S, H, F .} & =I_{z}, S_{z},(\delta+2 \gamma) \cos ^{2} \theta \\
& +(\delta-\gamma)\left(\sin ^{2} \theta \cos ^{2} \phi+\sin ^{2} \theta \sin ^{2} \phi\right) S_{z} I_{z} \\
& =I_{z}, S_{z},\left\{\delta+\gamma\left(3 \cos ^{2} \theta-1\right)\right\} \quad \ldots \ldots
\end{aligned}
$$

Equation (A-21) gives the spin Hamiltonian term for the hyperfine interaction for the case of $\vec{S}$ and $\vec{I}$ both being quantized along $\vec{H}, \delta$. and $\gamma$ are known as the isotropic and anisotropic hyperfine coupling constants, respectively. 


\section{APPENDIX II}

\section{SPIN HAMILTONIAN FOR $\mathrm{S}_{5 / 2}$ - STATE IONS IN A \\ CUBIC CRYSTALLINE FIELD}

Electron paramagnetism*occurs whenever an atomic system has a resultant angular momentum of electronic origin。 One way this can happen is for an ion to have a partially filled inner shell。 This happens in the case of the transition elements. For example, the Iron group elements $\left({ }_{21} \mathrm{Sc} \rightarrow{ }_{29} \mathrm{Cu}\right)$ have an electronic configuration consisting of a complete core, which is the same as the argon configuration, a partially filled $3 d$ shell, and two 4 s electrons (except copper which has only one $4 \mathrm{~s}$ electron). According to Hund's rule, ${ }^{56}$ whenever an electronic shell of an ion is half filled, the ground state of the Ion is an S-state. There are three commonly occuring tons in the iron group for which this happens: $\mathrm{Cr}^{+}, \mathrm{Mn}^{2+}$, and $\mathrm{Fe}^{3+}$, Each of these ions has a ${ }^{6} \mathrm{~S}_{5 / 2}$ ground state due to a (Ar)(3d) electronic configuration. If one of these paramagnetic ions is placed in a solid, it w111 see a crystalline field due to its neighboring lons in the solid. In general, the crystalline field destroys some of the orbital degeneracy of the ground state, Also, further splitting is caused by spin-orbit coupling $(\lambda \mathrm{L} \circ \mathrm{S})$, and there are magnetic interactions between the magnetic moment of the paramagnetic ion and neighboring magnetic moments in the lattice (electronic and nuclear).

In what follows consider only the specific example of an ${ }^{6} \mathrm{~S}_{5 / 2}$ Ion located substitutionally at a cation site in a cubic crystal. To first order, there is no splitting of the ground state since it is an orbital singlet. Higher order perturbation theory does give a splitting of the six magnetic sub-levels as is observed experimentally. The theory 
of the S-state Ion splitting is discussed in detall in references 11,14 , and 15 .

The Spin Hamiltonian used to describe the ${ }^{6} \mathrm{~S}_{5 / 2}$ ion will have the following terms: (1) the Zeeman interaction terms, (2) the hyperfine interaction, and (3) the fine structure term. The electronic Zeeman term 1s given by

$$
g B \vec{S} \cdot \vec{H}
$$

where

$$
\begin{aligned}
& g=\text { electronic } g \text { factor, } \\
& B=\text { Bohr magneton, and } \\
& \vec{H}=\text { external magnetic fleld. }
\end{aligned}
$$

The hyperfine interaction is described in detail in Appendix I. The fine structure term Incorporates the combined effects of the crystalline field, spin-orb1t coupling, and the spin-spin interaction between the electrons of the paramagnetic 1on. Hence, among other things the fine structure term will depend on the crystalline field and $\vec{S}$. This term will be dealt with in detall in the remainder of this appendix.

The general form of the fine structure term and how it depends on $\overrightarrow{\mathrm{S}}$ can be found by symmetry considerations. When a paramagnetic ion is placed in a solid 1ts. wavefunction w11l be determined by the crystalline. fleld. Therefore, in the absence of an external magnetic fleld the spin Hamiltonian which describes the paramagnetic Ion will have the symmetry of the crystalline field. Consider the ${ }^{6} \mathrm{~S}_{5 / 2}$ ion to be situated at a cubic site, which is taken as the origin of an $(x, y, z)$ coordinate system as shown in Fig. 27. The $x, y$, and $z$ axes lie along the [100], [010], and [001] crystaline directions, respectively; and they are three mutuaily orthogonal and equivalent axes. This implies that the fine structure term In the spin Hamiltonian must remain unchanged by any permutation of $(x, y, z)$ 。 


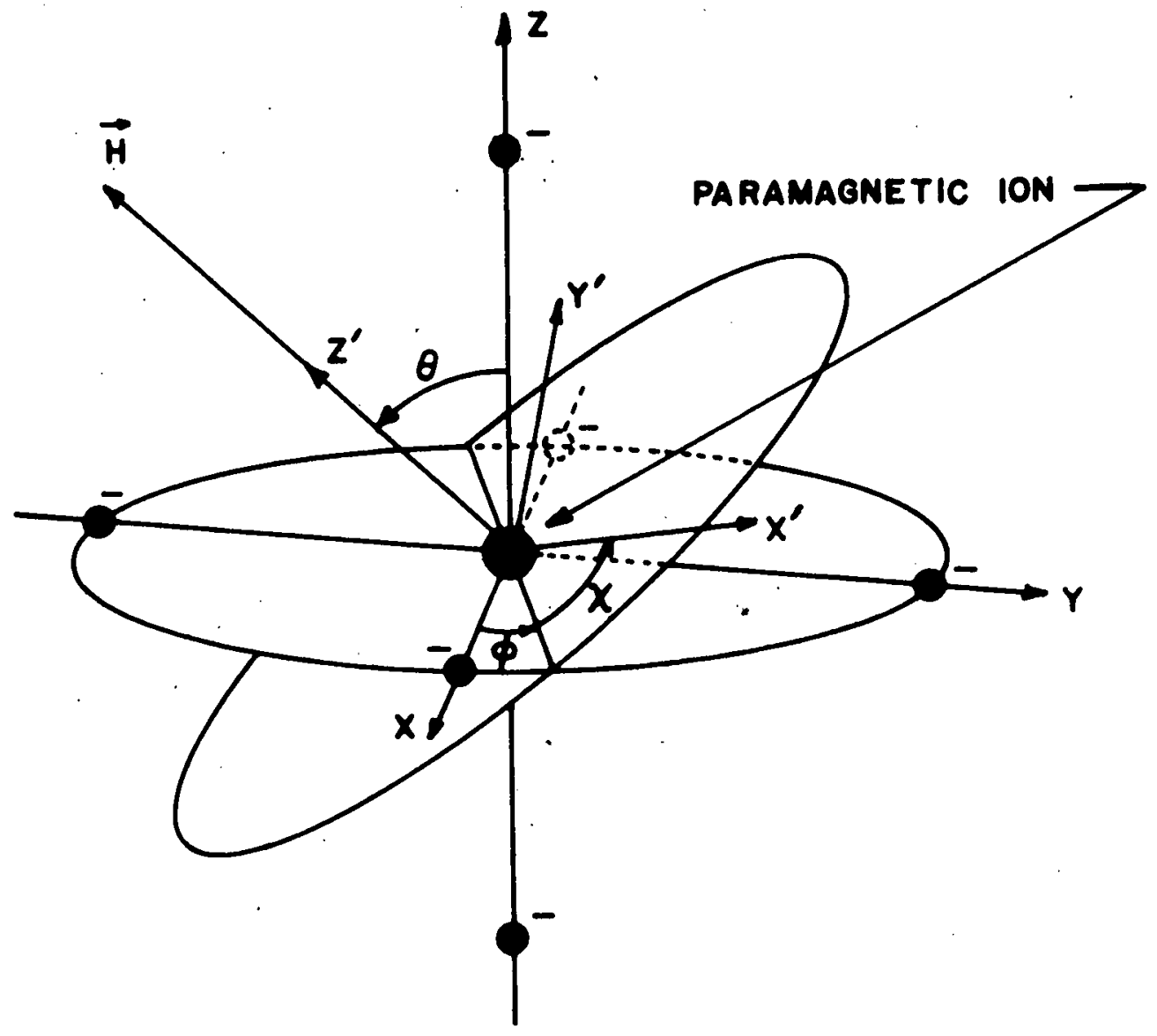

Fig. 27. Coordinate system of the cublc lattice $(x, y, z)$ and a primed coordinate system in which is along the $z^{\prime}$ axis. 
Also, if for example $\mathrm{s}_{\mathrm{x}}{ }^{2}$ appears in the spin Hamiltonian, then $\mathrm{s}_{\mathrm{y}}{ }^{2}$ and $\mathrm{s}_{\mathrm{z}}{ }^{2}$ must appear with the same coefficient, $i_{0} e_{0} c\left(\mathrm{~s}_{\mathrm{x}}{ }^{2}+\mathrm{s}_{\mathrm{y}}{ }^{2}+\mathrm{s}_{\mathrm{z}}{ }^{2}\right)$ 。 However, since $s_{x}^{2}+s_{y}{ }^{2}+s_{z}^{2}=s(s+1)$ is a constant, it can be neglected in the determination of the magnetic energy level structure. To find the most general spin dependence of the fine structure term all possible combinations of the spin components must be considered。 Terms such as $\mathrm{S}_{\mathrm{x}} \mathrm{S}_{\mathrm{y}}$ can be expressed as functions of $\mathrm{S}_{\mathrm{x}}{ }^{2}, \mathrm{~S}_{\mathrm{y}}{ }^{2}$, and $\mathrm{S}_{\mathrm{z}}{ }^{2}$ and therefore they can also be neglected. Other possibilities are terms like $\mathrm{S}_{\mathrm{x}}{ }^{2} \mathrm{~S}_{\mathrm{y}}{ }^{2}$ and $\mathrm{s}_{\mathrm{x}}{ }^{4}$. Note that

$$
\begin{aligned}
{[s(s+1)]^{2} } & =\left(s_{x}{ }^{2}+s_{y}{ }^{2}+s_{z}{ }^{2}\right)^{2}=s_{x}^{4}+s_{y}{ }^{4}+s_{z}{ }^{4} \\
& +\left(s_{x}{ }^{2} s_{y}{ }^{2}+s_{y}{ }^{2} s_{z}{ }^{2}+s_{z}{ }^{2} s_{x}{ }^{2}+s_{y}{ }^{2} s_{x}{ }^{2}+s_{z}{ }^{2} s_{y}{ }^{2}+s_{x}{ }^{2} s_{z}{ }^{2}\right)
\end{aligned}
$$

Hence terms such as $\mathrm{S}_{\mathrm{x}}{ }^{2} \mathrm{~S}_{\mathrm{y}}{ }^{2}$ can be replaced by some multiple of $\left(s_{x}^{4}+s_{y}^{4}+s_{z}^{4}\right)$ plus a constant。. For $s=5 / 2$, it can be shown that even power terms of sixth and higher orders reduce to expressions in $\mathrm{s}_{\mathrm{x}}{ }^{4}, \mathrm{~s}_{\mathrm{y}}{ }^{4}$, and $\mathrm{s}_{\mathrm{z}}{ }^{4}$ and constants. 57 odd power terms of the spin components do not appear because of symmetry considerations. For example a rotation of $90^{\circ}$ about the $z$ axis leaves the crystalline field unchanged. This means that a term with odd powers of the spin operators such as $c\left(\mathrm{~S}_{x}{ }^{3}+\mathrm{s}_{y}{ }^{3}+\mathrm{s}_{z}{ }^{3}\right)$ must remain unchanged under this operation。 But for this rotation $S_{x} \rightarrow S_{y}, S_{y} \rightarrow-S_{x}$, and $S_{z} \rightarrow S_{z}$. Therefore

$$
c\left(s_{x}^{3}+s_{y}^{3}+s_{z}^{3}\right) \rightarrow c\left(s_{y}^{3}-s_{x}^{3}+s_{z}^{3}\right)
$$

This implies that the constant $c=0$ in order for the spin Hamiltonian to remain unchanged. Hence the $\vec{S}$ dependence of the fine structure term takes the form: 


$$
\mathcal{H}_{\mathrm{S}_{0}, \mathrm{~F}_{\circ} \mathrm{S}_{\circ}}=\frac{1}{6} a\left[s_{x}^{4}+s_{y}^{4}+s_{z}^{4}-\frac{1}{5} s(s+1) \cdot\left(3 s^{2}+3 s-1\right)\right]
$$

where $a$ is the fine structure constant and the term in $S$ is a constant added for convenience in latter calculations. The fine structure constant depends on the crystalline field and all the parameters which characterize the particular ${ }^{6} \mathrm{~S}_{5 / 2}$ ion being described。

For convenience in latter calculations the expression for $\mathcal{H}_{S_{\vartheta}} F_{0} S_{0}$ given by $\mathrm{Eq} 。(\mathrm{~A}-24)$ can be restated in a form which depends only on $\mathrm{S}_{z}$ and $\left(s_{x} \pm 1 s_{y}\right)$. Let $s_{ \pm}=s_{x} \pm 1 s_{y}$ then

$$
s_{ \pm}^{-2}=s_{x}^{2}+s_{y}^{2} \pm 1\left(s_{x} s_{y}+s_{y} s_{x}\right)
$$

and

$$
\begin{aligned}
s_{ \pm}^{4} & =s_{x}^{4}+s_{y}^{4}-s_{x}{ }^{2} s_{y}{ }^{2}-s_{y}{ }^{2} s_{x}{ }^{2} \pm 1 s_{x}{ }^{2}\left(s_{x} s_{y}+s_{y} s_{x}\right) \\
& \pm 1\left(s_{x} s_{y}+s_{y} s_{x}\right) s_{x}{ }^{2} \mp 1 s_{y}{ }^{2}\left(s_{x} s_{y}+s_{y} s_{x}\right) \\
& \mp 1\left(s_{x} s_{y}+s_{y} s_{x}\right) s_{y}{ }^{2}-s_{x} s_{y} s_{x} s_{y}-s_{y} s_{x} s_{y} s_{x} \\
& -s_{x} s_{y}{ }^{2} s_{x}-s_{y} s_{x}{ }^{2} s_{y}
\end{aligned}
$$

However。

$$
\mathrm{s}_{\mathrm{x}}^{2} \mathrm{~s}_{\mathrm{y}}^{2}+\mathrm{s}_{\mathrm{y}}{ }^{2} \mathrm{~s}_{\mathrm{x}}^{2}=\left(\mathrm{s}_{\mathrm{x}}^{2}+\mathrm{s}_{\mathrm{y}}^{2}\right)^{2}-\mathrm{s}_{\mathrm{x}}^{4}-\mathrm{s}_{\mathrm{y}}^{4}
$$

The commutator of $S_{\ell}$ and $S_{m}$ is given by

$$
\left[S_{\ell D} S_{m}\right]=1 \varepsilon_{\ell m n} S_{n}
$$

where $S_{k}$ is a cartesian component of $\vec{S}$ with $k=1,2$; or 3 ; and $\varepsilon_{\ell m n}$ is the usual permutation operator。 Using Eqs。 $(A-26),(A-27)$, and $(A-28)$ It is found that 


$$
\begin{aligned}
s_{x}{ }^{4}+s_{y}{ }^{4} & =\frac{1}{8}\left(s_{+}^{4}+s_{-}^{4}\right)+\frac{3}{4}\left[s(s+1)-s_{z}{ }^{2}\right]^{2} \\
& +\frac{3}{4} s_{z}{ }^{2}-\frac{1}{2}\left[s(s+1)-s_{z}{ }^{2}\right]
\end{aligned}
$$

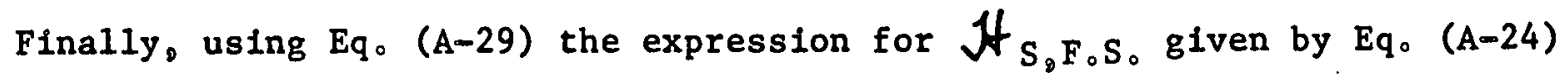
can be written as

$$
\begin{aligned}
\Psi_{S_{\nabla} F_{0} S_{0}} & =\frac{a}{120}\left[35 s_{z}{ }^{4}-30 s(s+1) s_{z}{ }^{2}+25 s_{z}{ }^{2}-6 s(s+1)\right. \\
& \left.+3 s^{2}(s+1)^{2}\right]+\frac{a}{48}\left[s_{+}{ }^{4}+s_{-\infty}{ }^{4}\right]
\end{aligned}
$$

The expectation values of $\mathrm{s}_{\mathrm{x}}{ }^{4}, \mathrm{~S}_{\mathrm{y}}{ }^{4}$, and $\mathrm{s}_{z}{ }^{4}$ depend on the direction of $\vec{H}$ relative to the crystalline axes. Hence, If $_{S_{\vartheta}} F_{0} S_{\circ}$ also depends on the direction of $\vec{H}_{0}$ This dependence can be calculated using Eq。(A-30)。 As shown in Fig。27 let the external magnetic field $\overrightarrow{\mathrm{H}}$ be along the $z^{\circ}$ axis of a coordinate system whose Euler angles are $(\theta, \phi, x)$ with respect to the $(x, y, z)$ system. The cholce of $x^{8}$ and $y^{\theta}$ is arbitrary and it will be seen that $x$ does not appear in the final result of the calculation. The components of $\vec{S}$ in the $\left(x^{8}, y^{\beta}, z^{0}\right)$ system are indicated by $S_{x}^{\theta}, S_{y}^{0}$ and $\mathbf{S}_{\mathbf{z}^{\circ}}^{0}$

As mentioned previously the fine structure is a higher than first order effect for S state lons. For typical S state lons it is found that the magnitude of the fine structure term in the spin Hamiltonian is much less than the magnitude of the Zeeman term for $H \sim 3000$ gauss。 15 Hence。 assume that the Zeeman term is the largest term. In the spin Hamiltonian for $H \sim 3000$ gauss. Therefore, if $\mathrm{g}$ is a scalar, $15 \overrightarrow{\mathrm{S}}$ is quantized along the direction of $\vec{H}_{\text {. }}$ In this case, the spin Hamiltonian should be transformed from the $(x, y, z)$ system to the coordinate system in which the Zeeman term is simply $\mathrm{gBS}_{z}^{0} \mathrm{H}_{\theta} 1_{0} e_{\circ}$, the $\mathrm{x}^{\theta}, \mathrm{y}^{\theta}, \mathrm{z}^{0}$ system。 Therefore, 


$$
\begin{aligned}
S_{z}= & \sin \theta \sin \chi S_{x}^{\theta}+\sin \theta \cos \chi S_{y}^{\theta}+\cos \theta S_{z}^{\theta} \\
= & \frac{\sin \theta}{2 i}\left(e^{i \chi}-e^{-i \chi}\right) S_{x}^{\theta}+\frac{\sin \theta}{2}\left(e^{i X}+e^{-i \chi}\right) S_{y}^{\theta}+\cos \theta S_{z}^{\theta} \\
= & -i \cos \frac{\theta}{2} \sin \frac{\theta}{2} e^{i x}\left(S_{x}^{\theta}+i S_{y}^{0}\right)+\left(\cos ^{2} \frac{\theta}{2}-\sin ^{2 \theta}\right) S_{z}^{\theta} \\
& +i \cos \frac{\theta}{2} \cdot \sin \frac{\theta}{2} e^{-i X}\left(S_{x}^{\theta}-i S_{y}^{\theta}\right) \\
= & \infty \sigma \zeta S_{+}^{0}+\left(\sigma \sigma^{*}-\zeta \zeta^{*}\right) S_{z}^{\theta}
\end{aligned}
$$

where

$$
\begin{aligned}
& \sigma=\cos \frac{\theta}{2} e^{1 / 2 i(\phi+x)} \\
& \tau=i \sin \frac{\theta}{2} e^{1 / 2 i(-\phi+x)} \\
& S_{ \pm}^{\theta}=S_{x}^{0} \pm i S_{y}^{0}
\end{aligned}
$$

Similarly,

$$
S_{+}=\sigma^{2} S_{+}^{\theta}+2 c \zeta^{*} S_{z}^{\theta}-\zeta^{* 2} S_{-}^{\theta} \quad
$$

and

$$
\mathrm{S}_{-}=\sigma^{* 2} \mathrm{~S}_{-}^{0}+2 \sigma^{*} \zeta \mathrm{S}_{\mathrm{z}}^{\theta}-\zeta^{2} \mathrm{~S}_{+}^{0} \text { 。 }
$$

Since it has been assumed that the fine structure term is much smaller than the Zeeman term, first order perturbation theory is sufficient to determine the changes in the energy level structure due to the fine structure term。 Hence。

$$
E\left(m_{S}^{0}\right)=g B H m_{S}^{0}+\left\langle S^{0}{ }_{\theta} m_{S}^{0}\left|\beta \psi_{S} F_{\circ} S_{\circ}\right| S^{\theta}{ }_{\theta} m_{S}^{\theta}\right\rangle \quad 。
$$

To evaluate the matrix element in $\mathrm{Eq}_{0}(\mathrm{~A}-37)_{\text {。 }}$ it is first necessary to find expressions for $\mathrm{S}_{z}{ }^{2}, \mathrm{~S}_{z}{ }^{4}, \mathrm{~S}_{+}{ }^{4}$, and $\mathrm{S}_{\infty}{ }^{4}$ in terms of the components of $\vec{S}^{0}$. This can be done by using Eqs。(A-31)。(A-35)。 and $(A-36)$ 。 Only the diagonal elements are necessary for this first order calculation. Bearing this in mind and using the fact that 


$$
\begin{aligned}
& s_{ \pm}^{0}\left|s^{0}{ }_{0} m_{s}^{0}\right\rangle=\sqrt{\left(S^{0} \bar{f} m_{s}^{0}\right)\left(S^{0} \pm m_{s}^{0}+1\right)}\left|s^{0}, m_{s}^{0} \pm 1\right\rangle, \\
& \sigma^{4} \zeta^{* 4}+\zeta^{4} \sigma^{* 4}=\frac{\sin \theta}{8} \cos 4 \phi \\
& \sigma \sigma^{*}-\zeta \zeta^{*}=\cos \theta \\
& \sigma \sigma^{*} \zeta \zeta^{*}=\frac{\sin ^{2} \theta}{4}
\end{aligned}
$$

1t can be shown that to lst order in $a_{\vartheta}$ the fine structure constant,

$$
\begin{aligned}
& E_{ \pm 1 / 2}= \pm \frac{g B H}{2}+p a \\
& E_{ \pm 3 / 2}= \pm \frac{3 g B H}{2}-\frac{3}{2} p a \\
& E_{ \pm 5 / 2}= \pm \frac{5 g B H}{2}+\frac{1}{2} p a
\end{aligned}
$$

For transitions given by $\Delta \mathrm{m}_{\mathrm{s}}= \pm I_{\text {。 }}$

$$
\begin{aligned}
& \Delta \mathrm{E}( \pm 1 / 2+\mp 1 / 2)=\mathrm{gBH} \\
& \Delta \mathrm{E}( \pm 3 / 2 \rightarrow \pm 1 / 2)=\mathrm{gBH} \mp 5 / 2 \mathrm{pa} \\
& \Delta \mathrm{E}( \pm 5 / 2 \rightarrow \pm 3 / 2)=\mathrm{gBH} \pm 2 \mathrm{pa}
\end{aligned}
$$

where

$$
p=\frac{1}{8}\left(35 \cos ^{4} \theta-30 \cos ^{2} \theta+3+5 \sin ^{2} \theta \cos 4 \phi\right)
$$

For a rotation about a [110] axis, $\phi=45^{\circ}$. The angular dependence of the fine structure lines for this case is shown in Fig。28。 In this figure $(-2.5 \mathrm{pa})$ is plotted as a function of the angle $\theta$. This curve gives the angular varfation of the resonance line due to the transition $m_{s}=3 / 2 \rightarrow 1 / 2$ (see Eq。(A-46))。The angular vartations of the resonance lines due to transitions $\mathrm{m}_{s}= \pm 5 / 2 \rightarrow \pm 3 / 2$ and $\mathrm{m}_{\mathrm{s}}=-3 / 2 \rightarrow-1 / 2$ are 


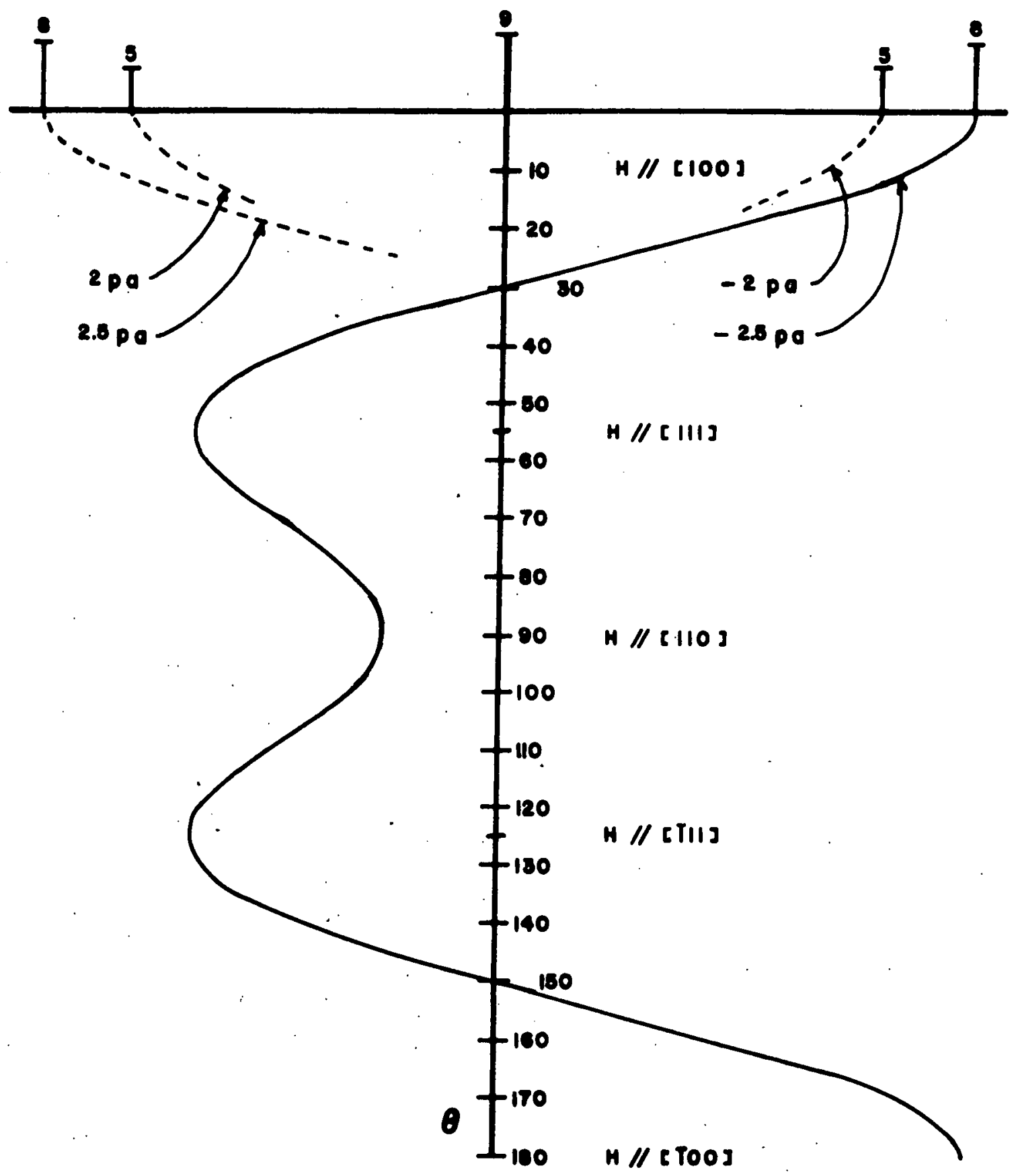

Fig. 28. Angular variation of the fine structure components of a resonance line for rotation about the [110] $\operatorname{ax1s}\left(\phi=45^{\circ}\right)$. 
similar to this variation and indicated by dashed lines in Fig。28. The transitions due to $m_{s}= \pm 1 / 2+\mp 1 / 2$ have no angular dependence as indicated by $\mathrm{Eq}$. $(\mathrm{A}-45)$. The lines at the top of the figure indicate the intensity ratios of the individual ines. These ratios will be discussed below. Note that the fine structure disappears when $p=0$ and this happens when $\theta \sim 30^{\circ}$. Also, the maximum separation of the fine structure Iines is when $\theta=0^{\circ}(\overrightarrow{\mathrm{H}} / /[100])$. When $\overrightarrow{\mathrm{H}} / /[110], \theta=90^{\circ}$ and there is a secondary minimum in the separation of the fine structure lines.

According to the "Golden Rule" of time dependent perturbation theory, 58 the Intensity of the lines given by Eqs. (A-45), (A-46), and $(A-47)$ are proportional to the square of the matrix element perturbation operator, $f^{\prime}$, taken between the two states differing by $\Delta m_{s}= \pm 1$ 。 However, $\mathcal{H}^{\prime}=-\vec{\mu}, 0 \overrightarrow{\mathrm{H}}^{\prime}(t)$ where $\vec{\mu}$ is the magnetic dipole operator of the paramagnetic Ion and $\vec{H}^{\prime}(t)$ is the microwave magnetic field which is perpendicular to $\vec{H}_{0}$ : Therefore, $f^{\prime}=-\mu_{x^{\prime}} H_{x^{\prime}}^{\prime}(t)$. Also,

$$
\left|<s, m_{s}\right| \mu_{x},\left.\left|s, m_{s}-1\right\rangle\right|^{2} \propto\left(s+m_{s}\right)\left(s-m_{s}+1\right)
$$

where $m_{s}$ is the magnetic quantum number of the upper level involved in the transition. This gives for an intensity rat1o

$$
I_{5 / 2}: I_{3 / 2}: I_{1 / 2}: I_{-1 / 2}: I_{-3 / 2}=5: 8: 9: 8: 5
$$

where $I_{m_{s}}$ is the intensity of the transition $\pm m_{s}+ \pm\left(m_{s}-1\right)$ 。

For cases. where the fine structure term is not too much smaller than the Zeeman term, terms of higher order in a must be considered. Detalls of these higher order corrections are given by Low。 32 


\section{LIST OF REFERENCES}

1. F. Seltz, Rev。Mod。Phys。26, 7 (1954)。

2. W.D. Compton and $H_{0}$ Rabin in Sol1d State Physics, eds。F。 Seitz and D. Turnbu11, Vol。 16 (Academ1c Press, New York, 1964)。

3. Ho Seldel and HoC,Wolf, Phys。Stat, Sol, 11, 3 (1965)。

4. H。 Plck, Erg。Exakt。Naturw1ss。38, 1 (1965)。

5. JoH. Schulman and W.D. Compton, Color Centers in Solids (MacMillan Co。. New York, 1962)。

6. E.U. Condon and Ho Odishaw, Eds. Handbook of Physics (McGraw-Hill, New York, 1958), po I-102。

7. Ho Seldel, Phys, Letters I, 27 (1963)。

8. W.D. Compton and $H_{0}$ Rabin, 1oc. c1t., Pp, 141-4, 148-51.

9. W.D. Compton and H。 Rabin, 10c. c1t. pp.146-7.

10. Jo Schulman and $W_{0} D$. Compton, 10c. cit., pp. 160-187.

11. G.E. Pake, Paramagnet1c Resonance (W.A. Benjamin, Inc, New York, 1962)。

12. C.P. Slichter, Principles of Magnetic Resonance (Harper \& Row, New York, 1963)。

13. Ao Abragam, The Princlples of Nuclear Magnet1sm (Clarendon Press, Oxford, 1961)。

14. S。A。Al'tshuler and B。M。Kozyrev, Electron Paramagnetic Resonance (Academ1c Press, New York, 1964)。

15. Wo Low, Paramagnetic Resonance In Sol1ds (Academic Press, New York, 1960)。

16. $D_{0} C_{0}$ Krupka and $R_{0} H_{0}$ Silsbee, Phys。Rev。 Letters 12, 193 (1964)。

17. H。 Seldel, et, al。, Z。 Phys。182, 398 (1965)。

18. W. Low, 10c. cito. p. 39.

19. Bausch and Lomb Reference Manual \#33-28-02 (Bausch and Lomb, Inc。, Rochester, New York)。

20. Varian Associates Publication $\$$ 87-100-074 (Varian Assoclates, Palo Alto, Callfornia)。 
21. Varian Associates Staff, NMR and EPR Spectroscopy,. (Pergamon Press, New York, 1960), pp。66-68。

22. $R_{0} A_{\circ}$ Andrews and $Y_{0} W_{0} K_{1 m}, R e v 。 S c 1$ Instr。 37, 411 (1966)。

23. R。Ager, $T$ 。 Cole, and J。Lambe, Rev。Sc1。Instr。34, 308 (1963)。

24. E。L。Ginzton, Microwave Measurements (McGraw-H111 Book Co。 Inc。 New York, 1957), Appendix A, po 480。

25. B。 Bleaney aud Wo Hayes, Proc。Phys。Soc。70B, 626 (1957)。

26. Wo Hayes and D。A。 Jones, Proc。Phys。Sco。 71, 503 (1958)。

27. M。 Satoh and C.P. S1ichter, Phys。Rev。 144, 259 (1966): These authors summarize studies of $\mathrm{Fe}^{3+}$ in $\mathrm{AgCl}$. All $\mathrm{Fe}^{3+}$ studies before 1958 are reported In Ref。14。 pp。 319 and 140。

28. S。A。A ${ }^{\circ}$ tshuler and BoM。 Kozyrev, loc。 cit。o pp。139-140。

29.W。Hayes, J。Appl。Phys。 Supp。to Vo1。33, p。 329 (1962)。

30。 D。H。 McMahon, Phys。Rev。134, A128 (1964)。

31. WoC。Holton and $\mathrm{H}_{0} \mathrm{~B}_{\mathrm{C}} \mathrm{um}_{2}$ Phys。Rev。 125,89 (1962): The F center resonance in $\mathrm{NaF}$ is an isotropic line at $\mathrm{g}=2.001$ with a partially resolved structure: 19 lines with $37.7 \mathrm{~g}$ separation。

32. Wo Low, loc. cit. 0 pp $114-117$.

33. L。H, Ahrens, Geochim。et, Cosmochim。Acta $\underline{2}, 155$ (1952)。

34. NoS。G11l and R。S。Hyholm, J。Chem。Soc。 3997 (1959)。

35. Wo Low, loc. c1tos p. 159。

36. Wo Low, 1oro c1tos po 118 。

37. S。A. $A 1^{\circ}$ tshuler and $B_{0} M$ 。 Kozyrev, 10c。 citos $p_{0} 72$ 。

38. HoWatanabe, Progr。Theoret, Phys。(Kyoto) 18, 405 (1957)。 Terms which are third order in $K$ are considered by Watanabe, but according to his theory the coefficients of these terms make their contribution almost negligible. This is not what is observed in the present work。

39. F。 Lifty。Z。Phys。165, 17 (1961)。

40。 E。 Link and F。 Lity, Abstract \#120, Int。 Symp。Color Centers in Alkali Halldes, Univ。 of Ill。, Urbana, Ill。(1965)。

41。 CoJ。Delbecq, Z。 Phys。171, 560 (1963)。 
42. Y。 Farge, $M_{0}$ Lambert, and $R_{0}$ Smoluchowski, Solid State Comm。 333 (1966)。

43. NoP。Kalabukhov and $P \circ K_{0}$ Gorbenko, Optika i Spektrosk。16, 475 (1964); [English translation in: Optics and Spectrosc。16. 258 (1964)]。

44. MoJ。Kirai, Phys。Soc。Japan 14, 1400 (1959)。

45... WoE。 Bron and A。S。Nowick, Phys。Rev。 119, 114 (1960)。

46. H。W. Etzel, Phys。 Rev。 87,906 (1952)。

47. G。J。 Dienes, J。Chem。Phys。 16, 620 (1948)。

48. R。A。 Andrews and $Y_{\circ} W_{\circ} \mathrm{Kim}_{8}$ Phys。 Letters 21, 616 (1966)。

49. S。Hattori。 J。Phys。Soc。Japan 17, 1454 (1962)。

50。 K。 Kubo, Jo Phys。 Soc。 Japan 16, 2294 (1961)。

51。S。Hattori。 J。Phys。Soc。Japan 15 2117 (1960)。

52. H。F。 Ivey, Phys。Rev。 72,341 (1947)。

53. R。Herman, M。C。Wallis, and R。F。Wallis, Phys。Rev。103, 87 (1956)。

54. C。B。Plerce, Phys。Rev。135, A83 (1964)。

55. W。 Low 10 ce cito 0 。58。

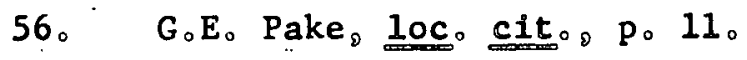

57. B。 Bleaney and $\mathrm{K}_{0} \mathrm{~W}_{\circ} \mathrm{H}_{0}$ Stevens, Repts。 Progr。in Phys。 16。 108 (1953)。

58. E。 Merzbacher, Quantum Mechanics ( $J$ 。Wiley \& Sons, Inc。 New York。 1961)。 Po 470 。 